\title{
Investigations of the Application of CFD to Flow Expected in the Lower Plenum of the Prismatic VHTR
}

Richard W. Johnson

Donna P. Guillen

Tara Gallaway

September 2006

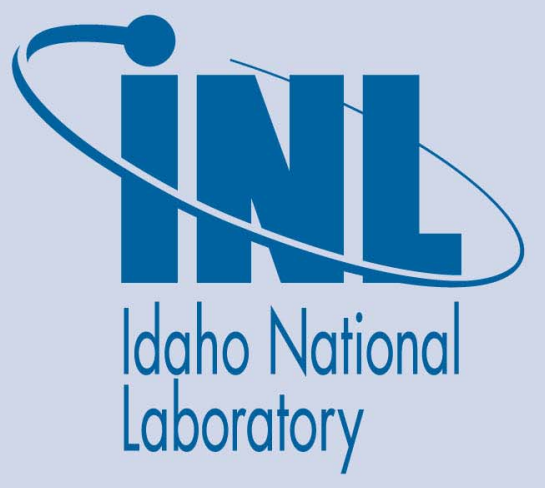

The INL is a U.S. Department of Energy National Laboratory operated by Battelle Energy Alliance 
INL/EXT-06-11756

\title{
Investigations of the Application of CFD to Flow Expected in the Lower Plenum of the Prismatic VHTR
}

\author{
Richard W. Johnson \\ Donna P. Guillen \\ Tara Gallaway
}

September 2006

Idaho National Laboratory
Idaho Falls, Idaho 83415

Prepared for the

U.S. Department of Energy

Office of Nuclear Energy

Under DOE Idaho Operations Office

Contract DE-AC07-05ID14517 
This page intentionally left blank. 


\section{Executive Summary}

The Generation IV (Gen IV) very high temperature reactor (VHTR) will either be a prismatic (block) or pebble bed design. However, a prismatic VHTR reference design, based on the General Atomics Gas Turbine-Modular Helium Reactor (GT-MHR) [General Atomics, 1996] has been developed for preliminary analysis purposes [MacDonald, et al., 2003]. Numerical simulation studies reported herein are based on this reference design. In the lower plenum of the prismatic reference design, the flow will be introduced by dozens of turbulent jets from the core above. The jet flow will encounter rows of columns that support the core. The flow from the core will have to turn ninety degrees and flow toward the exit duct as it passed through the forest of support columns. Due to the radial variation of the power density in the core, the jets will be at various temperatures at the inlet to the lower plenum. This presents some concerns, including that local hot spots may occur in the lower plenum. This may have a deleterious effect on the materials present as well as cause a variation in temperature to be present as the flow enters the power conversion system machinery, which could cause problems with the operation of the machinery.

In the past, systems analysis codes have been used to model flow in nuclear reactor systems. It is recognized, however, that such codes are not capable of modeling the local physics of the flow to be able to analyze for local mixing and temperature variations. This has led to the determination that computational fluid dynamic (CFD) codes be used, which are generally regarded as having the capability of accurately simulating local flow physics. Accurate flow modeling involves determining appropriate modeling strategies needed to obtain accurate analyses. These include determining the fineness of the grid needed, the required iterative convergence tolerance, which numerical discretization method to use, and which turbulence model and wall treatment should be employed. It also involves validating the computer code and turbulence model against a series of separate and combined flow phenomena and selecting the data used for the validation. This report describes progress made to identify proper modeling strategies for simulating the lower plenum flow for the task entitled "CFD software validation of jets in crossflow," which was designed to investigate the issues pertaining to the validation process.

The flow phenomenon previously chosen to investigate is flow in a staggered tube bank because it is shown by preliminary simulations to be the location of the highest turbulence intensity in the lower plenum. Figure ES-1 shows preliminary computations of flow in the lower plenum and the exit hot duct, colored by turbulence intensity from a plan view. The commercial CFD code FLUENT ${ }^{\mathbb{B}}$ was used to make the simulations.

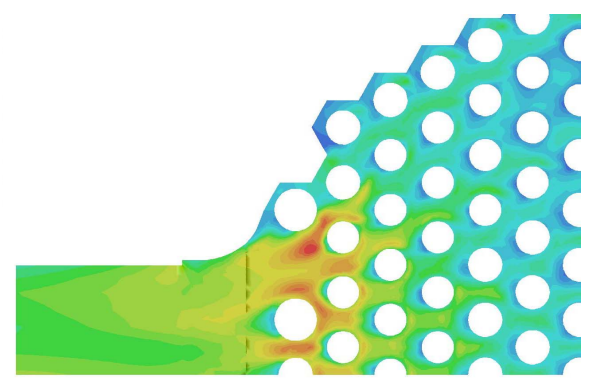

Figure ES-1. Contours of turbulence intensity in the lower plenum showing a plan view of flow toward the exit duct.

The flow geometry for flow in tube banks for which there are experimental data is given by Figure ES-2. The flow entering at the left is set to the same values as the flow exiting at the right. The same is true for the top and bottom. 


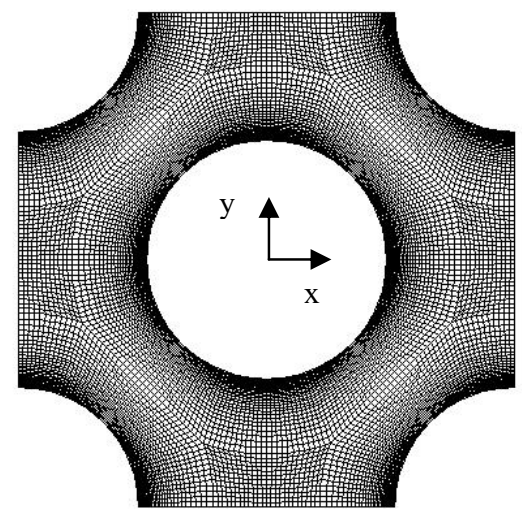

Figure ES-2. Geometry and grid used to investigate flow in a staggered tube bundle.

Numerical simulations were previously obtained assuming that the flow shown in Figure ES-2 is steady. Various turbulence models were employed along with strategies to reduce numerical error to allow appropriate comparisons of the results. It was determined that the sophisticated Reynolds stress model (RSM) provided the best results. It was later determined that the flow of Figure ES-2 is an unsteady flow wherein circulating eddies grow behind the tube and 'peel off' alternately from the top and the bottom of the tube. Additional calculations show that the mean velocity is well predicted when the flow is modeled as an unsteady flow. Figure ES-3 compared steady (red) results with unsteady (blue) results against experimental data (green symbols). The results for $U$ at the left are clearly superior for the unsteady computations; the unsteady computations for the turbulence stress on the right are similar to those for the steady calculations, showing the same trends. It is clear that strategies must be employed to reduce numerical errors before comparing simulation results to the data and that the correct physics must be simulated to achieve reasonable agreement between simulations and experiment.

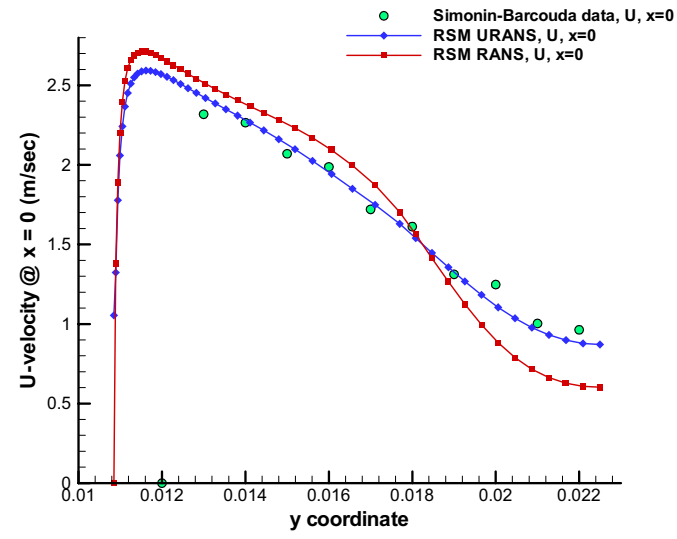

(a)

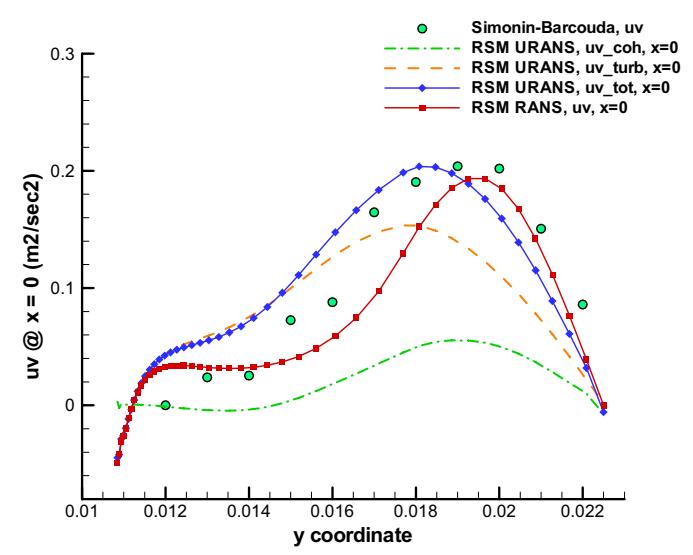

(b)

Figure ES-3. Computations for the (a) mean velocity in the $\mathrm{x}$-direction and (b) the turbulent shear stress for $\mathrm{x}=0$ for steady and unsteady simulations.

In addition to the above numerical studies, this report details preliminary efforts to model a larger region of the lower plenum, consisting of an array of five cylindrical support posts and half-posts in a confined channel with flow entering through 4 inlet jets above the plenum. Figure ES-4 illustrates a 
model of the flow region. The 4 inlet jet ports on the top of the model are clearly visible; the flow exits the model to the right through an extended outlet. The progress to date for the CFD predictions of flow through a flow test model representing a section of the VHTR lower plenum is reported herein. Information obtained from these simulations will be used for the final CFD solutions and to guide experimentation. Experimental data obtained in the Idaho National Laboratory Matched-Index-ofRefraction facility will comprise a benchmark to assess CFD models for applicability to analyze flow in the VHTR lower plenum. Two CFD codes, FLUENT ${ }^{\circledR}$ and NPHASE, were run for the identical geometry and inlet conditions. NPHASE employs a steady-state Reynolds-Averaged Navier-Stokes (RANS) computation with a $\mathrm{k} \sim \varepsilon$ turbulence model. Unsteady RANS (URANS) computations with a $\mathrm{k} \sim \varepsilon$ turbulence model were run using FLUENT ${ }^{\circledR}$. The final set of experimental data was not available in time to be incorporated into the inlet profiles, so assumed data profiles were used. Once the final data is available, the methodology is in place to use actual test data. This report presents preliminary results from the CFD computations, including velocity, pressure, and turbulence quantities. Figure ES-5 shows preliminary results for the velocity magnitude along the mid-plane of the model. Analysis of results and recommendations for the experimental setup are also given.

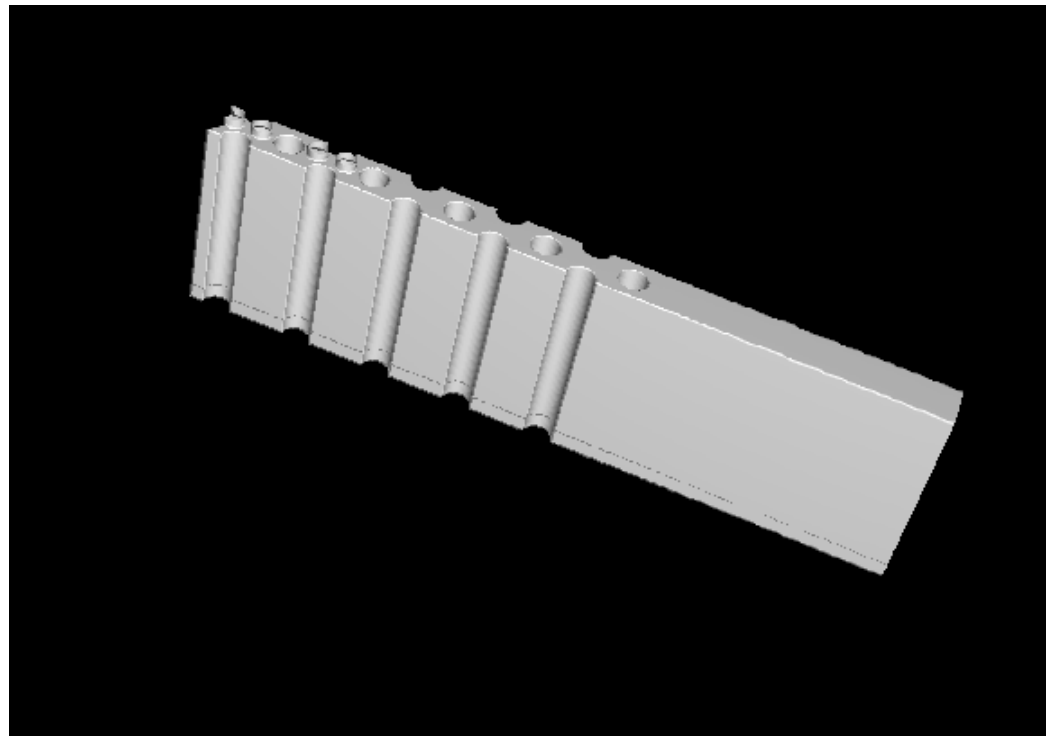

Figure ES-4. Illustration of the flow region from the prismatic reference VHTR design lower plenum.

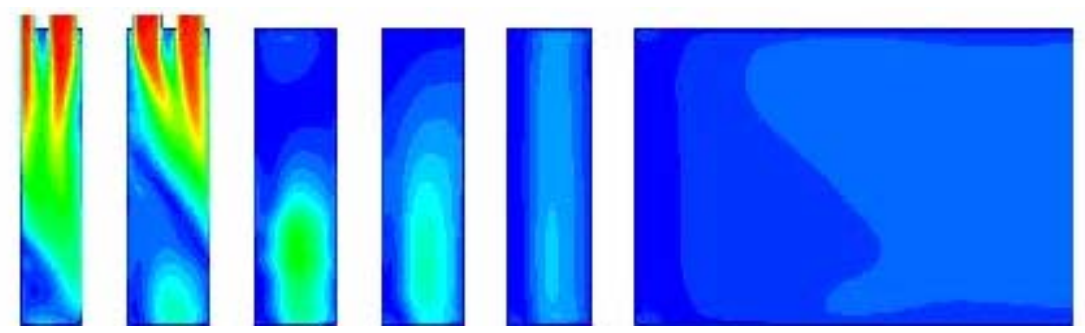

Figure ES-5. Preliminary results for the velocity magnitude along the mid-plane of the model. 
This page intentionally left blank. 


\section{Contents}

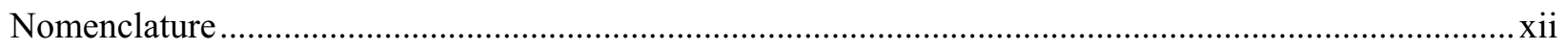

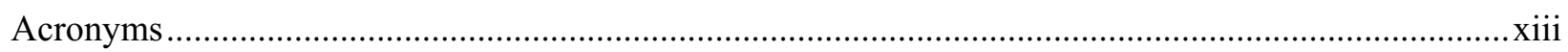

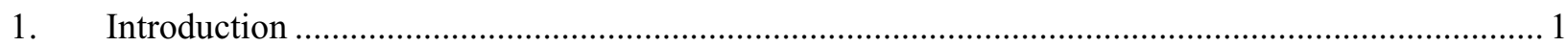

2. Part 1: Validation Studies of CFD Software …............................................................................ 2

2.1 Layout of the VHTR Reactor Vessel .............................................................................. 2

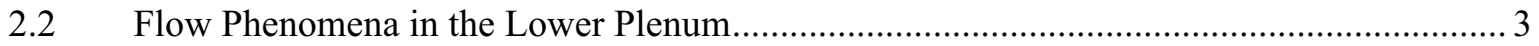

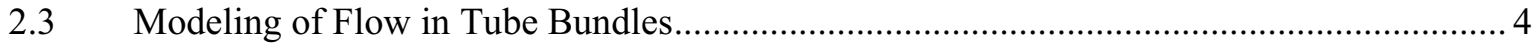

2.4 Steady State Calculations of Flow in Tube Bundles ....................................................... 9

2.5 Unsteady Calculations of Flow in Tube Bundles ........................................................ 13

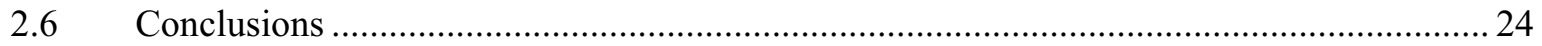

3. Part 2: Preliminary CFD Simulations of the VHTR Lower Plenum …….................................... 25

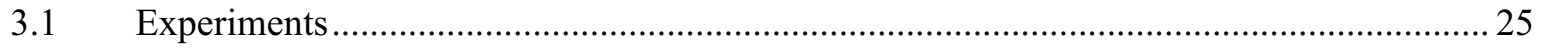

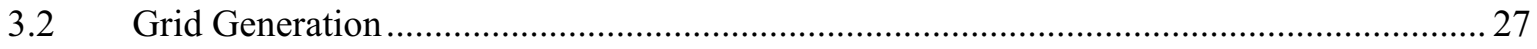

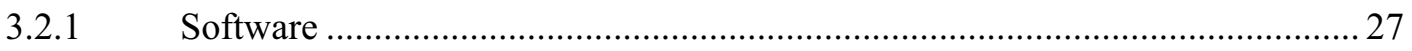

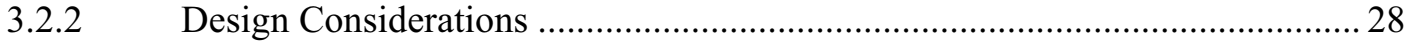

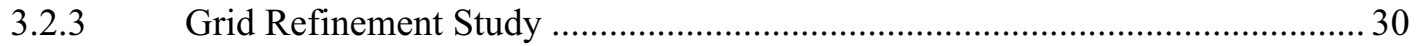

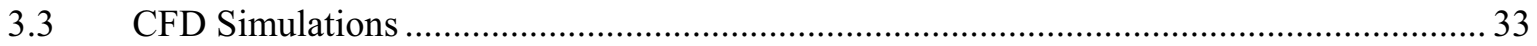

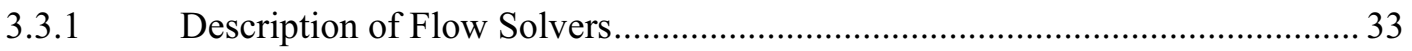

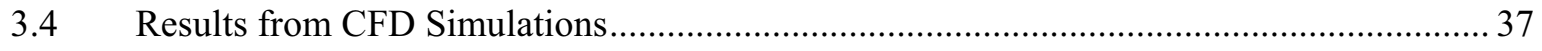

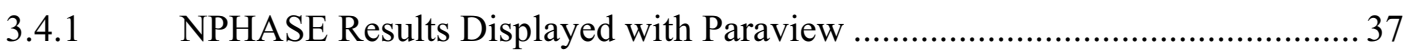

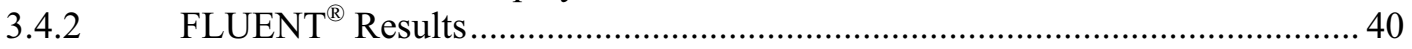

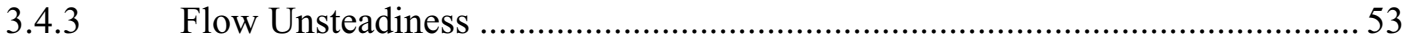

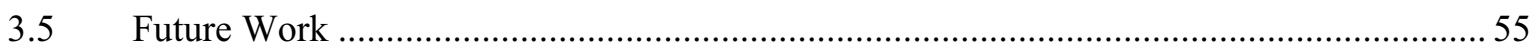

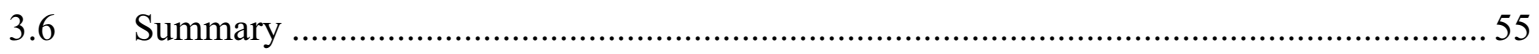

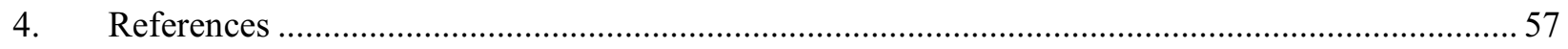

Appendix: User-Defined Function (UDF) to Compute Time-Averaged Correlations ..............................6 60 


\section{Figures}

Figure ES-1. Contours of turbulence intensity in the lower plenum showing a plan view of flow toward the exit duct.

Figure ES-2. Geometry and grid used to investigate flow in a staggered tube bundle. iv

Figure ES-3. Computations for the (a) mean velocity in the $\mathrm{x}$-direction and (b) the turbulent shear stress for $\mathrm{x}=0$ for steady and unsteady simulations.

Figure ES-4. Illustration of the flow region from the prismatic reference VHTR design lower plenum.... V

Figure ES-5. Preliminary results for the velocity magnitude along the mid-plane of the model. . V

Figure 1. Layout of the power conversion system (PCS) and reactor vessel for the gas-cooled prismatic VHTR reference design [courtesy of General Atomics (GA)]. 2

Figure 2. Graphite fuel blocks showing (a) the TRISO coated fuel particle in the fuel compacts placed in the hex block shown in perspective and (b) plan views (courtesy of General Atomics)...... 3

Figure 3. Flow phenomena of lower plenum showing (a) path lines of coolant entering the lower plenum and (b) contours of turbulence intensity in the lower plenum in a plan view of the flow near the exit duct.

Figure 4. FLUENT ${ }^{\circledR}$ grid for the tube bundle simulations (a) and $\mathrm{y}^{+}\left(\equiv \mathrm{u}_{\tau} \mathrm{y} / \mathrm{v}\right)$ distribution for the grid (b).10

Figure 5. Comparison of solutions for iterative tolerances of $1 \times 10^{-3}$ to $1 \times 10^{-6}$ for (a) the mean streamwise velocity $U$ and (b) the normal stress $\overline{v^{2}}$ at $\mathrm{x}=0$.

Figure 6. The B-spline fitted to the data of Simonin and Barcouda for $U$ at $\mathrm{x}=0$ compared to results for the $\mathrm{k} \sim \varepsilon$ model for standard and nonequilibrium wall functions and the enhanced wall treatment... 11

Figure 7. Mean velocity profiles at $\mathrm{x}=0$ for realizable $\mathrm{k} \sim \varepsilon, \mathrm{v}^{2}-\mathrm{f}$ and RSM models.

Figure 8. Results for (a) $\overline{u^{2}}$ and (b) $\overline{v^{2}}$ normal stresses and (c) the shear stress $\overline{u v}$ for the realizable $\mathrm{k} \sim \varepsilon$ model using the enhanced wall treatment, the $v^{2}-f$ model, and the RSM using wall functions at $\mathrm{x}=$ 0 .

Figure 9. (a) The instantaneous quantity $u$ for a point in space and over a period of time, along with (b) the ensemble average $\langle u\rangle$ of the signal and (c) the (turbulent) fluctuating component $u^{\prime}$

Figure 10. The (a) time- and ensemble- averaged quantity and (b) the non-turbulent fluctuating quantity.15

Figure 11. Time plots of the ensemble average velocity at two points in the flow for 250 steps.

Figure 12. URANS results compared to steady RANS results for $x=0$ for (a) the mean streamwise velocity $U$, (b) the mean transverse velocity $V$, and the time averaged Reynolds stresses, including the coherent and turbulent correlations and their total: (c) the streamwise normal, (d) the transverse normal and (e) the cross-correlation, plus (f) the profile location superimposed on the finer grid. . 19 
Figure 13. URANS results compared to steady RANS results for $x=11 \mathrm{~mm}$ for (a) the mean streamwise velocity $U$, (b) the mean transverse velocity $V$, and the time averaged Reynolds stresses, including the coherent and turbulent correlations and their total: (c) the streamwise normal, (d) the transverse normal and (e) the cross-correlation, plus (f) the profile location superimposed on the finer grid.. 20

Figure 14. URANS results compared to steady RANS results for $\mathrm{x}=16.5 \mathrm{~mm}$ for (a) the mean streamwise velocity $U$, (b) the mean transverse velocity $V$, and the time averaged Reynolds stresses, including the coherent and turbulent correlations and their total: (c) the streamwise normal, (d) the transverse normal and (e) the cross-correlation, plus (f) the profile location superimposed on the finer grid.

Figure 15. URANS results compared to steady RANS results for $y=0$ for (a) the mean streamwise velocity $U$, (b) the mean transverse velocity $V$, and the time averaged Reynolds stresses, including the coherent and turbulent correlations and their total: (c) the streamwise normal, (d) the transverse normal and (e) the cross-correlation, plus (f) the profile location superimposed on the finer grid. . 22

Figure 16. URANS results compared to steady RANS results for $y=22.5 \mathrm{~mm}$ for (a) the mean streamwise velocity $U$, (b) the mean transverse velocity $V$, and the time averaged Reynolds stresses, including the coherent and turbulent correlations and their total: (c) the streamwise normal, (d) the transverse normal and (e) the cross-correlation, plus (f) the profile location superimposed on the finer grid.

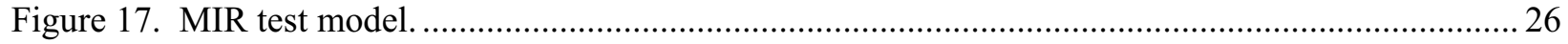

Figure 18. Flow test model dimensions (a) top view, and (b) front view. ........................................... 27

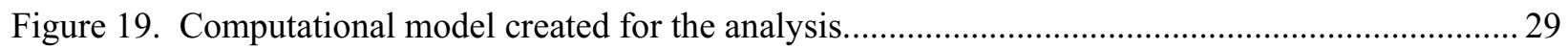

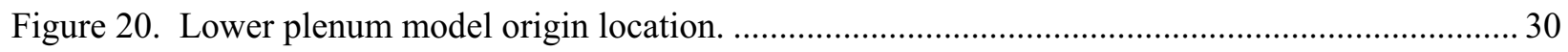

Figure 21. Detail sections of meshes created for grid independence study using FLUENT ${ }^{\circledR}$.................. 32

Figure 22. Detail section of hybrid grids created for grid independence study using NPHASE. .............. 32

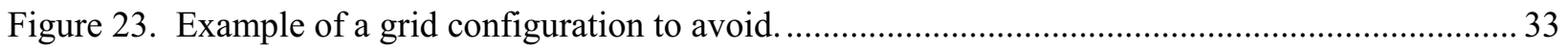

Figure 24(a). Top view of inlet jets \#1 through 4 - contours of velocity magnitude. ............................... 36

Figure 24(b). Top view of inlet jets \#1 through 4 - contours of TKE ................................................... 36

Figure 25. Pressure and velocity distributions assuming constant inlet velocity and turbulence quantities

for the "medium" grid.

Figure 26. Pressure and velocity distributions assuming constant inlet velocity and turbulence quantities

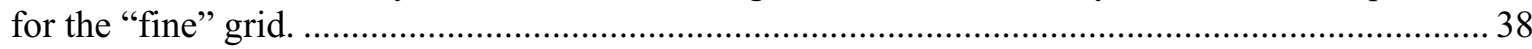

Figure 27. Pressure and velocity distributions using experimental profiles for inlet velocity and turbulence quantities for the "medium" grid.

Figure 28(a). Pressure and velocity distributions using experimental profiles for inlet velocity and turbulence quantities for the "fine" grid using NPHASEe. 
Figure 28(b). Pressure and velocity distributions using experimental profiles for inlet velocity and turbulence quantities for the "fine" grid using FLUENT®.

Figure 29. Computed contours of $\mathrm{x}$-velocity on the $\mathrm{x}-\mathrm{y}$ plane along the centerline $(\mathrm{z}=0)$.

Figure 30. Computed contours of $\mathrm{y}$-velocity on the $\mathrm{x}-\mathrm{y}$ plane along the centerline $(\mathrm{z}=0)$................... 42

Figure 31. Computed contours of $\mathrm{z}$-velocity on the $\mathrm{x}-\mathrm{y}$ plane along the centerline $(\mathrm{z}=0) \ldots \ldots \ldots \ldots \ldots \ldots \ldots . . . . . . . . . .33$

Figure 32. Computed contours of velocity magnitude on the $\mathrm{x}-\mathrm{y}$ plane along the centerline $(\mathrm{z}=0) \ldots \ldots \ldots . . .43$

Figure 33. Computed contours of static pressure along the $x-y$ plane on the centerline $(z=0) \ldots \ldots \ldots \ldots \ldots . . . .44$

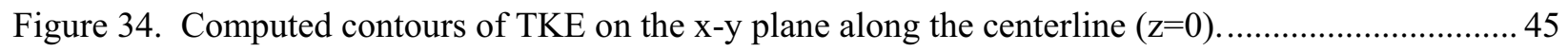

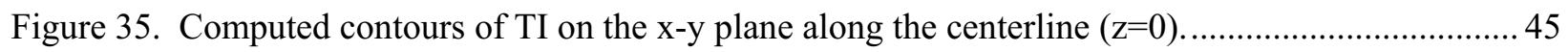

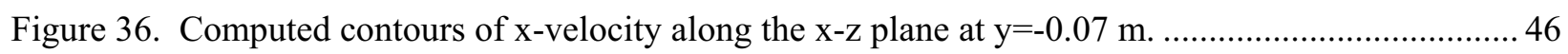

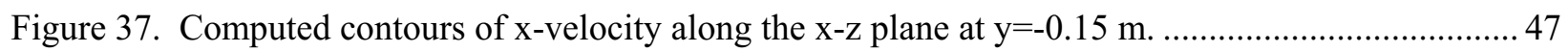

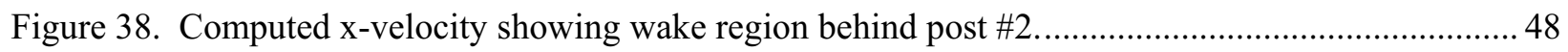

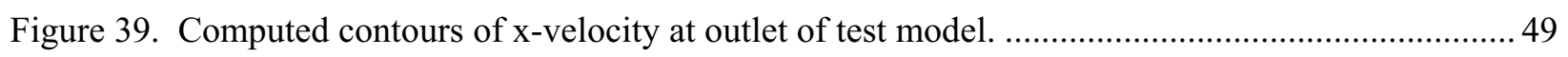

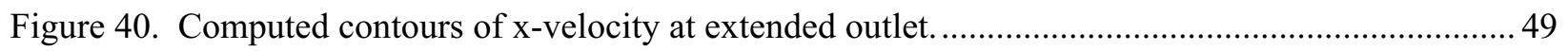

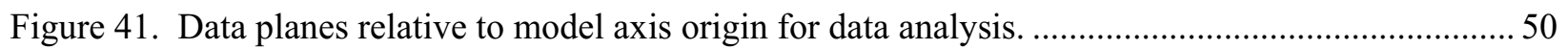

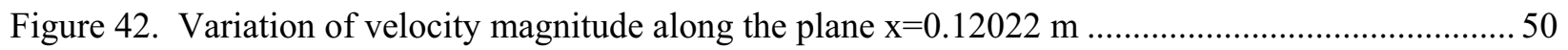

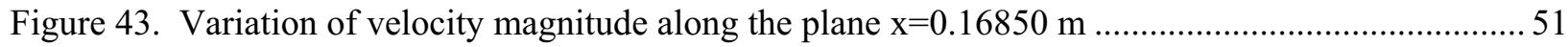

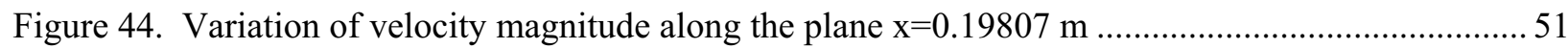

Figure 45. Variation of velocity magnitude along the plane $\mathrm{x}=0.26729 \mathrm{~m}$. .....................................52

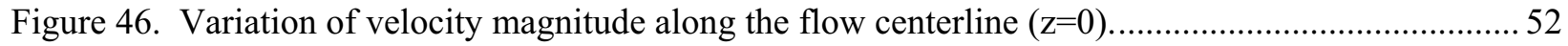

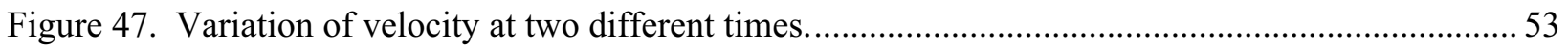

\section{Tables}

Table 1. Average global cell size for the computational meshes generated for this study...................... 33

Table 2. Minimum and maximum values in the VHTR lower plenum flow simulation. ........................ 40

Table 3. Computed maximum and minimum velocities present at test section and computational outlets

("fine" unstructured grid). 


\section{Nomenclature}

\begin{tabular}{|c|c|}
\hline $\mathrm{C}$ & modeling coefficient [dimensionless] \\
\hline $\mathrm{d}$ & diameter of support post $[\mathrm{m}]$ \\
\hline $\mathrm{h}$ & average global cell size $[\mathrm{m}]$ \\
\hline$f$ & body force $[\mathrm{N}]$ \\
\hline$k$ & turbulence kinetic energy $\left[\mathrm{m}^{2} / \mathrm{s}^{2}\right]$ \\
\hline$p$ & pressure $[\mathrm{Pa}]$ \\
\hline$P$ & mean pressure $[\mathrm{Pa}]$ \\
\hline $\mathrm{P}$ & production of turbulent kinetic energy $\left[\mathrm{kg} /\left(\mathrm{m} \mathrm{s}^{2}\right)\right]$ \\
\hline $\operatorname{Re}$ & Reynolds number \\
\hline St & Strouhal number \\
\hline$t$ & time $[\mathrm{s}]$ \\
\hline$u$ & $\mathrm{x}$-direction velocity component $[\mathrm{m} / \mathrm{s}]$ \\
\hline$u_{i}$ & velocity vector $[\mathrm{m} / \mathrm{s}]$ \\
\hline$v$ & $\mathrm{y}$-direction velocity component $[\mathrm{m} / \mathrm{s}]$ \\
\hline $\mathrm{V}_{\mathrm{x}}$ & $\mathrm{x}$-direction velocity component $[\mathrm{m} / \mathrm{s}]$ \\
\hline $\mathrm{V}_{\mathrm{y}}$ & $\mathrm{y}$-direction velocity component $[\mathrm{m} / \mathrm{s}]$ \\
\hline $\mathrm{V}_{\mathrm{z}}$ & z-direction velocity component $[\mathrm{m} / \mathrm{s}]$ \\
\hline$w$ & z-direction velocity component $[\mathrm{m} / \mathrm{s}]$ \\
\hline $\mathrm{W}_{\mathrm{v}}$ & vortex shedding frequency $[\mathrm{Hz}]$ \\
\hline$x_{i}$ & coordinate vector $[\mathrm{m}]$ \\
\hline $\mathrm{y}^{+}$ & normalized wall coordinate \\
\hline$\delta_{i j}$ & Kronecker delta tensor [dimensionless] \\
\hline$\varepsilon$ & turbulence dissipation rate $\left[\mathrm{m}^{2} / \mathrm{s}^{3}\right]$ \\
\hline$\varepsilon_{i j}$ & turbulence dissipation rate tensor $\left[\mathrm{m}^{2} / \mathrm{s}^{3}\right]$ \\
\hline
\end{tabular}


$\mu$

$\mu_{t}$

v

$\sigma$

$\rho$

$\tau_{w}$

angle brackets $(\langle u\rangle)$

prime $\left(u^{\prime}\right)$

double prime $\left(u^{\prime \prime}\right)$

$\operatorname{overbar}\left(\overline{u_{i}^{\prime} u_{j}^{\prime}}\right)$

capitalization $(U)$ dynamic viscosity $[\mathrm{kg} /(\mathrm{m} \mathrm{s})]$

dynamic turbulence viscosity $[\mathrm{kg} /(\mathrm{m} \mathrm{s})]$

kinematic viscosity $[\mathrm{m} 2 / \mathrm{s}]$

modeling coefficient [dimensionless]

density $[\mathrm{kg} / \mathrm{m} 3]$

wall shear stress $[\mathrm{N} / \mathrm{m} 2]$

ensemble averaged quantity

turbulent fluctuating quantity

fluctuating (turbulent plus coherent) quantity

time averaged quantity

time- and ensemble-averaged quantity

\section{Acronyms}

ASME

CFD

DNS

Gen IV

GT-MHR

INL

LES

MIR

MUSCL

PCS

PISO

PIV
American Society of Mechanical Engineers

Computational fluid dynamics

direct numerical simulation

Generation IV

Gas Turbine-Modular Helium Reactor

Idaho National Laboratory

large eddy simulation

Matched-Index-of-Refraction

Monotone Upstream-Centered Schemes for Conservation Laws

power conversion system

Pressure-Implicit with Splitting of Operators

Particle image velocimetry 
PRESTO PREssure STaggering Option

QUICK Quadratic Upstream Interpolation for Convective Kinematics

RANS Reynolds-Averaged Navier-Stokes

RMS Root-mean-square

RSM Reynolds Stress Model

SIMPLE Semi-Implicit Pressure Linked Equations

SIMPLEC SIMPLE-Consistent

TI Turbulent intensity [\%]

TKE Turbulent kinetic energy $\left[\mathrm{m}^{2} / \mathrm{s}^{2}\right]$

TRISO tri-isotopic

UDF user-defined function

URANS Unsteady Reynolds-Averaged Navier-Stokes

VHTR Very High Temperature Reactor 


\section{Investigations of the Application of CFD to Flow Expected in the Lower Plenum of the Prismatic VHTR}

\section{Introduction}

The Generation IV (Gen IV) very high temperature reactor (VHTR) will either be a prismatic (block) or pebble bed design. However, a prismatic VHTR reference design, based on the General Atomics Gas Turbine-Modular Helium Reactor (GT-MHR) [General Atomics, 1996] has been developed for preliminary analysis purposes [MacDonald, et al., 2003]. Numerical simulation studies reported herein are based on this reference design. In the lower plenum of the prismatic reference design, the flow will be introduced by dozens of turbulent jets from the core above. The jet flow will encounter rows of columns that support the core. The flow from the core will have to turn ninety degrees and flow toward the exit duct as it passed through the forest of support columns. Due to the radial variation of the power density in the core, the jets will be at various temperatures at the inlet to the lower plenum. This presents some concerns, including that local hot spots may occur in the lower plenum. This may have a deleterious effect on the materials present as well as cause a variation in temperature to be present as the flow enters the power conversion system machinery, which could cause problems with the operation of the machinery.

In the past, systems analysis codes have been used to model flow in nuclear reactor systems. It is recognized, however, that such codes are not capable of modeling the local physics of the flow to be able to analyze for local mixing and temperature variations. This has led to the determination that computational fluid dynamic (CFD) codes be used, which are generally regarded as having the capability of accurately simulating local flow physics. Accurate flow modeling involves determining appropriate modeling strategies needed to obtain accurate analyses. These include determining the fineness of the grid needed, the required iterative convergence tolerance, which numerical discretization method to use, and which turbulence model and wall treatment should be employed. It also involves validating the computer code and turbulence model against a series of separate and combined flow phenomena and selecting the data used for the validation. This report describes progress made to identify proper modeling strategies for simulating the lower plenum flow for the task entitled "CFD software validation of jets in crossflow," which was designed to investigate the issues pertaining to the validation process. 


\section{Part 1: Validation Studies of CFD Software}

\subsection{Layout of the VHTR Reactor Vessel}

The prismatic VHTR reference design, based on the General Atomics GT-MHR, is illustrated in Figure 1. The power conversion system (PCS) is shown to the left of the reactor. Helium coolant moves through the annulus of the hot duct as it returns from the PCS and annularly up the vessel wall of the reactor vessel to the upper plenum. The coolant then moves downward through the fueled portion of the reactor core and into the lower plenum. The heated coolant flows out of the lower plenum and through the center of the hot duct back to the PCS to complete the cycle. The core has an annular layout with an inner and an outer reflector as well as upper and lower reflectors (graphite blocks shown as white blocks in Figure 1). There are 102 heated graphite columns in the annular core. Each heated column has a stack of ten heated blocks plus additional unheated blocks above and below the heated ones.

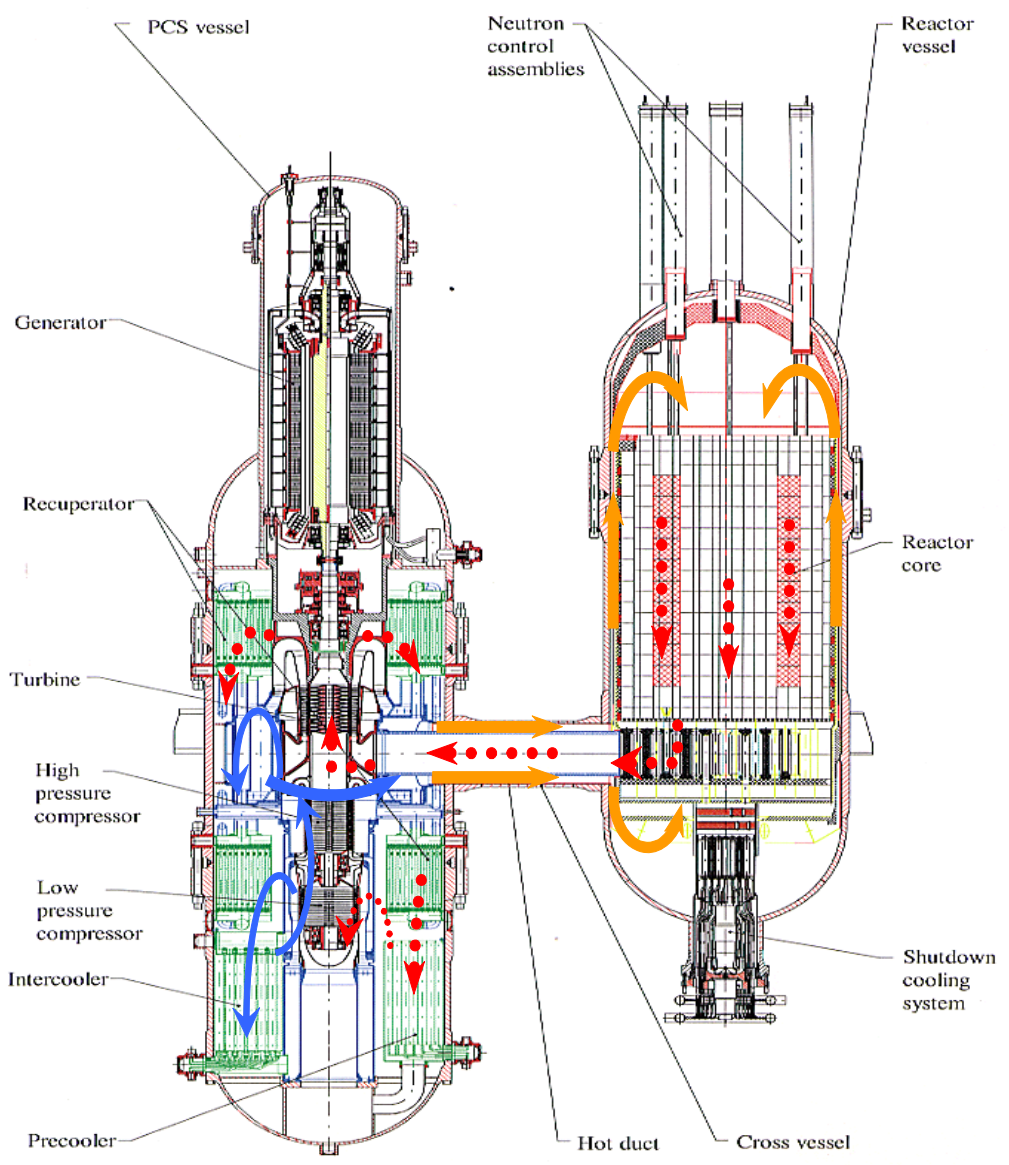

Figure 1. Layout of the power conversion system (PCS) and reactor vessel for the gas-cooled prismatic VHTR reference design [courtesy of General Atomics (GA)].

The fuel is contained in small ( $\sim 1 \mathrm{~mm}$ dia.) spherical particles called TRISO particles, which are then compacted into fuel pins. Figure 2 illustrates the TRISO particles, the fuel compacts, and the graphite blocks with the fuel pins inserted from both a perspective and a plan view. The plan view illustrates the layout of the fuel pins (blue) and the surrounding coolant channels (red). The fuel pins are $12.45 \mathrm{~mm}$ in diameter while the coolant channel diameters are 15.88 and $12.70 \mathrm{~mm}$ in diameter. Some of the blocks contain holes for the control rods, Figure 2a. 


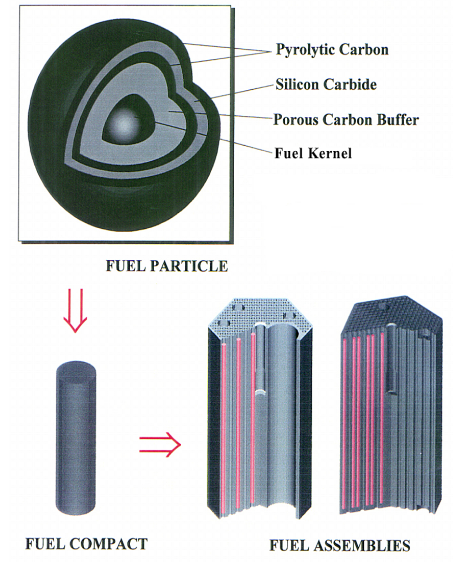

(a)

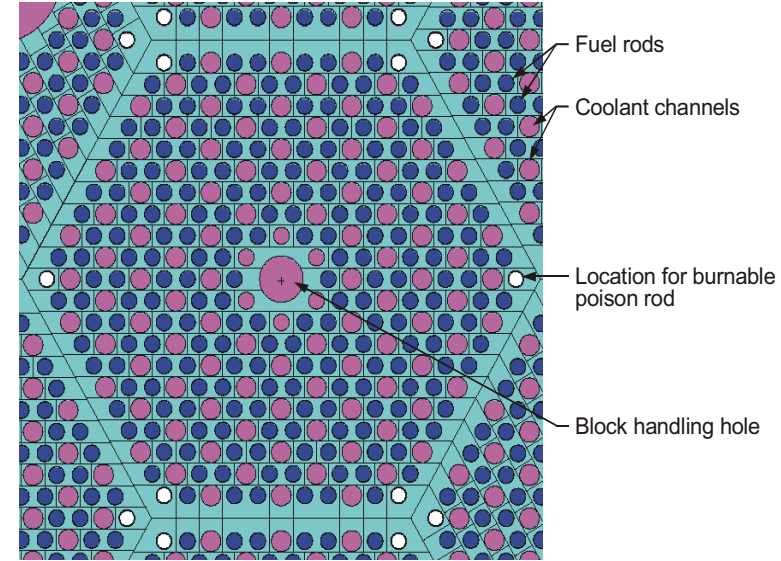

(b)

Figure 2. Graphite fuel blocks showing (a) the TRISO coated fuel particle in the fuel compacts placed in the hex block shown in perspective and (b) plan views (courtesy of General Atomics).

\subsection{Flow Phenomena in the Lower Plenum}

The hundreds of coolant channels in the VHTR core combine into several dozen larger ducts just before entering the lower plenum. The lower plenum is populated with dozens of columns to support the graphite blocks in the core. These columns will affect the jets as they enter into the plenum. As the coolant enters the lower plenum, it will be turned ninety degrees to flow towards the exit duct; hence, there will be a cross-flow of coolant in the lower plenum. The cross-flow will have to negotiate the support columns and will also experience an expanding flow area on the side away from the exit duct and a converging area as it approaches the exit duct. Figure 3a illustrates a preliminary calculation of path lines of the coolant in the lower plenum colored by temperature, showing the entering jets. Figure $3 \mathrm{~b}$ shows contours of turbulence intensity for a plan view of flow near the exit duct. The turbulence intensity is greatest in the converging flow region close to the exit duct.

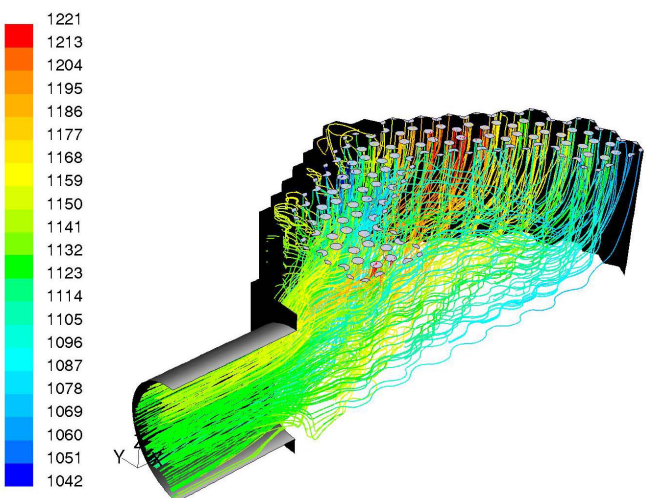

(a)

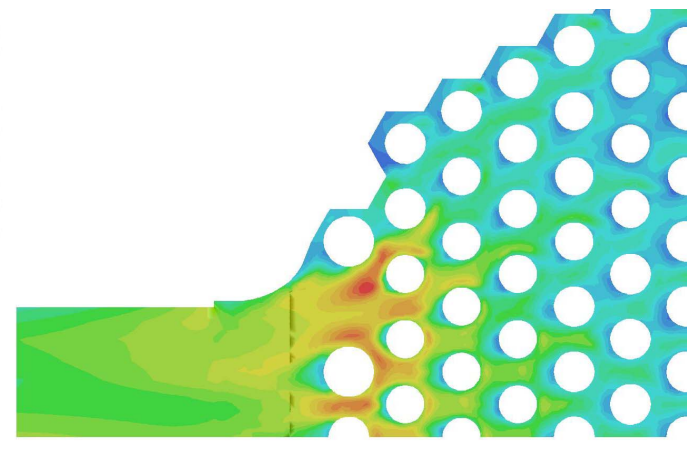

(b)

Figure 3. Flow phenomena of lower plenum showing (a) path lines of coolant entering the lower plenum and (b) contours of turbulence intensity in the lower plenum in a plan view of the flow near the exit duct.

The preliminary flow calculations illustrated in Figure 3 provide insight into the flow phenomena in the lower plenum. As the flow enters the lower plenum proper, the jets will not only have some interaction with each other but also with the support columns, which are located fairly close together as 
shown in Figure 3b. The location of a jet will have a significant effect on its interaction with the crossflow. Farther away from the exit duct, the cross-flow will be weaker. Hence, these jets will penetrate deeper into the lower plenum than those closer to the exit duct.

The cross-flow will have similarities to flow in tube banks. However, the flow coming straight across from the exit duct will see flow in staggered tube banks, while flow coming from wider angles will be closer to flow through in-line tube banks. The flow in the lower plenum will apparently be turbulent as may be deduced from the fact that the predicted turbulence intensity is relatively large for the preliminary calculations above. The flow will probably be unsteady due to vortex shedding behind the support columns.

The staggered flow in a tube bank of Simonin and Barcouda [1986, 1988] was chosen for comparison with simulations by Johnson [2005]. This report extends the validation studies of Johnson [2005] to include results for unsteady computations. The flow configuration of Simonin and Barcouda [1986] is of water through a staggered array of tubes $21.7 \mathrm{~mm}$ in diameter, each row spaced $45 \mathrm{~mm}$ apart on tube centers in both the streamwise and lateral directions. The bulk velocity ahead of the tube array is reported to be $1.06 \mathrm{~m} / \mathrm{sec}$. Data are reported for the mean velocities in the streamwise and transverse directions and the normal and shear Reynolds stresses for several locations. The Simonin and Barcouda data are available on the ERCOFTAC database ${ }^{\mathrm{a}}$. The data are provided for comparing turbulence model results and have been used for comparison with numerical simulations (e.g., Benhamadouche and Laurence [2003], Rollet-Miet, et al. [1999], Hassan and Barsamian [2004], and Moulinec, et al. [2004]).

\subsection{Modeling of Flow in Tube Bundles}

The flow in the lower plenum has been determined to be incompressible flow. (The Mach number is about 0.03 for normal operation). Flow in the tube banks of Simonin and Barcouda is incompressible and isothermal. The continuity and Navier-Stokes equations for incompressible, constant density, flow, given in tensor notation, are [Wilcox, 1994]:

$$
\begin{gathered}
\frac{\partial u_{i}}{\partial x_{i}}=0 \\
\rho \frac{\partial u_{i}}{\partial t}+\rho u_{j} \frac{\partial u_{i}}{\partial x_{j}}=-\frac{\partial p}{\partial x_{i}}+\frac{\partial}{\partial x_{j}}\left[\mu\left(\frac{\partial u_{i}}{\partial x_{j}}+\frac{\partial u_{j}}{\partial x_{i}}\right)\right]+\rho f_{i}
\end{gathered}
$$

where:
$u_{i}$ is the velocity vector
$p$ is pressure
$\rho$ is density
$\mu$ is the dynamic viscosity
$t$ is time
$x_{i}$ are the spatial coordinates.

Eq. (2) represents three equations, one each for conservation of momentum in each of the three Cartesian coordinate directions $\left(x=x_{1}, y=x_{2} \& z=x_{3}\right)$. For convenience, Eq. (2) can be rewritten using Eq. (1) as:

\footnotetext{
${ }^{\mathrm{a}}$ http://cfd.me.umist.ac.uk/ercoftac/
} 


$$
\rho \frac{\partial u_{i}}{\partial t}+\rho \frac{\partial u_{i} u_{j}}{\partial x_{j}}=-\frac{\partial p}{\partial x_{i}}+\frac{\partial}{\partial x_{j}}\left[\mu\left(\frac{\partial u_{i}}{\partial x_{j}}+\frac{\partial u_{j}}{\partial x_{i}}\right)\right]+\rho f_{i}
$$

In general, these equations are time-dependent. The traditional approach to solving these equations for turbulent flow is to perform Reynolds averaging of the equations. Turbulence itself is threedimensional and unsteady. However, if there are only turbulent fluctuations present, a turbulent flow is stationary; that is, the mean flow is steady. In this case, Reynolds averaging is accomplished by performing a time average of the flow equations. The length of the time average should be sufficient to ensure that the mean quantities so defined do not vary from time interval to time interval. Reynolds averaging is preceded by a decomposition of the dependent variables of velocity, pressure, and temperature (for nonisothermal flow) into a mean quantity and a (turbulent) fluctuating quantity, e.g.:

$$
u_{i}=U_{i}+u_{i}^{\prime}
$$

where:

$u_{i}$ is the instantaneous velocity

$U_{i}$ is the mean value

$u_{i}^{\prime}$ is the fluctuating component.

Reynolds decomposition is applied to the governing equations, Eqs. (1, 3), which are then time-averaged yielding the Reynolds-averaged Navier-Stokes (RANS) equations. In particular, the second term on the left-hand side of Eq. (3) becomes

$$
\rho \frac{\partial u_{i} u_{j}}{\partial x_{j}}=\rho \frac{\partial}{\partial x_{j}}\left[\left(U_{i}+u_{i}^{\prime}\right)\left(U_{j}+u_{j}^{\prime}\right)\right]=\rho \frac{\partial}{\partial x_{j}}\left[U_{i} U_{j}+u_{i}^{\prime} U_{j}+u_{j}^{\prime} U_{i}+u_{i}^{\prime} u_{j}^{\prime}\right] \text {. }
$$

Upon time averaging, the two middle terms above disappear because they are identically zero by the definition of time-averaging. With an overbar representing a time-averaged term, the RANS equations are:

$$
\rho \frac{\partial U_{i}}{\partial t}+\rho \frac{\partial}{\partial x_{j}}\left[U_{i} U_{j}+\overline{u_{i}^{\prime} u_{j}^{\prime}}\right]=-\frac{\partial P}{\partial x_{j}}+\frac{\partial}{\partial x_{j}}\left[\mu\left(\frac{\partial U_{i}}{\partial x_{j}}+\frac{\partial U_{j}}{\partial x_{i}}\right)\right]+\rho f_{i}
$$

The time-dependent term on the left-hand side is retained for convenience, though it is zero for a stationary flow. Turbulence modelers noticed that the new term $\rho \overline{u_{i}^{\prime} u_{j}^{\prime}}$ could be moved to the right side of the equation and joined with the diffusion term. This new term represents the action of turbulent fluctuations, which could be described as 'turbulent diffusion.' Hence, the typical forms of the Reynoldsaveraged continuity and Navier Stokes equations are given by

$$
\frac{\partial U_{i}}{\partial x_{i}}=0
$$




$$
\rho \frac{\partial U_{i}}{\partial t}+\rho U_{j} \frac{\partial U_{i}}{\partial x_{j}}=-\frac{\partial P}{\partial x_{j}}+\frac{\partial}{\partial x_{j}}\left[\mu\left(\frac{\partial U_{i}}{\partial x_{j}}+\frac{\partial U_{j}}{\partial x_{i}}\right)-\rho \overline{u_{i}^{\prime} u_{j}^{\prime}}\right]+\rho f_{i}
$$

The new term is called the Reynolds stress term. The Reynolds stresses are:

$$
-\rho \overline{u_{i}^{\prime} u_{j}^{\prime}} \text { for } i=1,2,3 ; j=1,2,3 \text {, }
$$

for constant density, where the indices $i$ and $j$ each represent the three spatial coordinates. The Reynolds stresses are the elements of a symmetric second rank tensor, which is comprised of six independent quantities. The shear stresses are given when $i \neq j$; the normal stresses are given for $i=j$. For essentially two-dimensional flow, there are only three non-zero stresses of interest, $-\rho \overline{u^{\prime} v^{\prime}},-\rho \overline{u^{\prime 2}}$ and $-\rho \overline{v^{\prime 2}}$. The Reynolds stresses are, in fact, not stresses in the normal sense of the definition. They are called stresses because they are grouped with the (molecular) viscous stresses in the RANS equations for convenience and for modeling purposes. They actually represent the turbulent transport of momentum, that is, the net turbulent transport in the ' $j^{\text {th }}$ ' direction of the ' $i$ 'th' momentum. To be able to solve the RANS equations, the Reynolds stresses must be modeled using a turbulence model.

One of the most common approaches to model the Reynolds stresses is called the Boussinesq eddy viscosity approach [Wilcox, 1994]. The eddy viscosity model assumes that the turbulence can be modeled using the rate of strain tensor multiplied by a scalar turbulent or eddy viscosity, e.g.:

$$
-\rho \overline{u_{i}^{\prime} u_{j}^{\prime}}=\mu_{t}\left(\frac{\partial U_{i}}{\partial x_{j}}+\frac{\partial U_{j}}{\partial x_{i}}\right)-\frac{2}{3} \rho k \delta_{i j}, \text { for } i=1,2,3 ; j=1,2,3,
$$

where:

$\mu_{t}$ is the turbulent (eddy) viscosity,

$k$ is the turbulent kinetic energy

$\delta_{i j}$ is the Kronecker delta.

In eddy viscosity models, the normal stresses are intrinsically assumed to be isotropic (equal) and are equal to $2 / 3$ of the turbulent kinetic energy, $\mathrm{k}$. Results for eddy viscosity approaches for the flow in the tube bundle of Simonin and Barcouda [1986] have been obtained and reported in Johnson [2005].

The most sophisticated approach using the RANS equations is to solve differential transport equations for each independent Reynolds stress, avoiding the eddy viscosity philosophy altogether. Such transport equations can be derived from the Navier-Stokes equations. If one takes the dot product of the velocity vector $u_{j}$ and the tensor NS equation, Eq. (1), for $u_{i}$ and adds to this product the reverse (the dot product of $u_{i}$ with the tensor NS equation for $u_{j}$ ), and then performs the Reynolds decomposition and takes the time average, one obtains the Reynolds stress transport equations. These equations include some terms that are exact and some that must be modeled. Because a separate equation is solved for each Reynolds stress, the model is, in general, anisotropic in terms of the normal stresses. That is, $\overline{u^{\prime 2}}, \overline{v^{\prime 2}}$ and $\overline{w^{\prime 2}}$ are not all equal, as assumed by eddy viscosity models. However, this approach requires more computation time because from four to seven differential equations must be solved, depending upon the dimensionality of the problem. 
Computations are reported herein using the Reynolds stress turbulence model available in

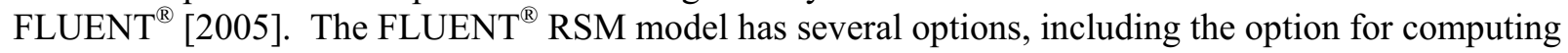
an extra equation for the turbulent kinetic energy to obtain boundary conditions for the Reynolds stresses. This option is a default option but is disabled as it seems inconsistent with using k computed with the $\mathrm{RSM}$; it was also found to prevent convergence to $10^{-6}$ tolerance, probably because of the inconsistency. Other than the aforementioned option, all other default options are used. The incompressible RSM in FLUENT $^{\circledR}$ used in the present study is based on the Reynolds stress transport equations given (using angle brackets to indicate Reynolds averaging) as:

$$
\frac{\partial}{\partial t}\left(\rho\left\langle u_{i}^{\prime} u_{j}^{\prime}\right\rangle\right)+C_{i j}=D_{T, i j}+\frac{\partial}{\partial x_{k}}\left(\mu \frac{\partial}{\partial x_{k}}\left\langle u_{i}^{\prime} u_{j}^{\prime}\right\rangle\right)+P_{i j}+\Phi_{i j}-\varepsilon_{i j}
$$

where:

$$
C_{i j}=\frac{\partial}{\partial x_{k}}\left(\rho\left\langle u_{k}\right\rangle\left\langle u_{i}^{\prime} u_{j}^{\prime}\right\rangle\right) \text { and } P_{i j}=-\rho\left(\left\langle u_{i}^{\prime} u_{k}^{\prime}\right\rangle \frac{\partial\left\langle u_{j}\right\rangle}{\partial x_{k}}+\left\langle u_{j}^{\prime} u_{k}^{\prime}\right\rangle \frac{\partial\left\langle u_{i}\right\rangle}{\partial x_{k}}\right) \text {. }
$$

The turbulent diffusion and pressure-strain terms are modeled, respectively, as

$$
D_{T, i j}=-\frac{\partial}{\partial x_{k}}\left(\frac{\mu_{t}}{\sigma_{k}} \frac{\partial\left\langle u_{i}^{\prime} u_{j}^{\prime}\right\rangle}{\partial x_{k}}\right) \text { and } \Phi_{i j}=\Phi_{i j, 1}+\Phi_{i j, 2}+\Phi_{i j, w}
$$

where:

$$
\begin{gathered}
\Phi_{i j, 1}=-C_{1} \rho \frac{\varepsilon}{k}\left(\left\langle u_{i}^{\prime} u_{j}^{\prime}\right\rangle-\frac{2}{3} \delta_{i j} k\right), \quad \Phi_{i j, 2}=-C_{2}\left[\left(P_{i j}-C_{i j}\right)-\frac{2}{3} \delta_{i j}(P-C)\right] \text { and } \\
\Phi_{i j, w}=C_{1}^{\prime} \frac{\varepsilon}{k}\left(\left\langle u_{k}^{\prime} u_{m}^{\prime}\right\rangle n_{k} n_{m} \delta_{i j}-\frac{3}{2}\left\langle u_{i}^{\prime} u_{k}^{\prime}\right\rangle n_{j} n_{k}-\frac{3}{2}\left\langle u_{j}^{\prime} u_{k}^{\prime}\right\rangle n_{i} n_{k}\right) \frac{k^{3 / 2}}{C_{\ell} \varepsilon d} \\
+C_{2}^{\prime}\left(\Phi_{k m, 2} n_{k} n_{m} \delta_{i j}-\frac{3}{2} \Phi_{i k, 2} n_{j} n_{k}-\frac{3}{2} \Phi_{j k, 2} n_{i} n_{k}\right) \frac{k^{3 / 2}}{C_{\ell} \varepsilon d}
\end{gathered}
$$

with:

$$
\begin{aligned}
& \sigma_{\mathrm{k}}=0.82 \\
& C_{1}=1.8 \\
& C_{2}=0.6 \\
& P=1 / 2 P_{k k} \\
& C=1 / 2 C_{k k} \text { (summation over repeated indices) } \\
& C_{1}{ }^{\prime}=0.5 \\
& C_{2}{ }^{\prime}=0.3 .
\end{aligned}
$$

$n_{k}$ is the $x_{k}$ component of the unit normal to the wall, $d$ is the normal distance to the wall, and $C_{\ell}=C_{\mu}^{3 / 4} / \kappa$, where $C_{\mu}=0.09, \mu_{t}=\rho C_{\mu} k^{2} / \varepsilon$, and $\kappa=0.4187$. The turbulent kinetic energy is given as $1 / 2\left\langle u_{i}^{\prime} u_{i}^{\prime}\right\rangle$ and the 
dissipation rate tensor $\varepsilon_{i j}=2 / 3 \delta_{i j} \rho \varepsilon$ for incompressible flow. The scalar dissipation rate $\varepsilon$ is found by solving its transport equation:

$$
\frac{\partial(\rho \varepsilon)}{\partial t}+\frac{\partial\left(\rho \varepsilon u_{i}\right)}{\partial x_{i}}=\frac{\partial}{\partial x_{j}}\left[\left(\mu+\frac{\mu_{t}}{\sigma_{\varepsilon}}\right) \frac{\partial \varepsilon}{\partial x_{j}}\right]+C_{\varepsilon 1} \frac{1}{2} P_{i i} \frac{\varepsilon}{k}-C_{\varepsilon 2} \rho \frac{\varepsilon^{2}}{k}
$$

where:

$$
\begin{aligned}
& \sigma_{\varepsilon}=1.0 \\
& C_{\varepsilon 1}=1.44 \\
& C_{\varepsilon 2}=1.92 .
\end{aligned}
$$

The wall treatment used for all of the computations reported is the standard wall function approach $\left[\right.$ FLUENT $\left.^{\circledR}, 2005\right]$ wherein the mean velocity in the wall-adjacent cell is calculated as:

$$
U^{*}= \begin{cases}\frac{1}{\kappa} \ln \left(E y^{*}\right) & \mathrm{y}^{*}>11.225 \\ \mathrm{y}^{*} & \mathrm{y}^{*}<11.225\end{cases}
$$

where:

$$
U^{*} \equiv \frac{U_{P} C_{\mu}^{1 / 4} k_{P}^{1 / 2}}{\tau_{w} / \rho}, \text { and } y^{*} \equiv \frac{\rho y_{P} C_{\mu}^{1 / 4} k_{P}^{1 / 2}}{\mu}
$$

and $\kappa=0.4187 ; E=9.793 ; U_{P}, k_{P}$ and $y_{P}$ are the mean velocity; and turbulence kinetic energy and wall distance at point $\mathrm{P}$ and $\tau_{w}$ is the wall shear stress. However, the near-wall nodes around the circumference of the tubes for the present calculations are within a ${ }^{*}$ value of 5, well within the viscous sublayer. (Attempts to use the enhanced wall treatment in FLUENT with the RSM were not convergent). The boundary values for the Reynolds stresses are given as $\left[\right.$ FLUENT $\left.^{\circledR}, 2005\right]$ :

$$
\frac{\left\langle u_{\tau}^{\prime 2}\right\rangle}{\tau_{w} / \rho}=5.1, \frac{\left\langle u_{\eta}^{\prime 2}\right\rangle}{\tau_{w} / \rho}=1.0, \frac{\left\langle u_{\lambda}^{\prime 2}\right\rangle}{\tau_{w} / \rho}=2.3, \text { and } \frac{-\left\langle u_{\tau}^{\prime} u_{\eta}^{\prime}\right\rangle}{\tau_{w} / \rho}=1.0
$$

where $\tau, \eta$, and $\lambda$ are the wall tangential, normal, and binormal coordinates, respectively.

Periodic boundary conditions are used for all open flow boundaries as was the case for Benhamadouche and Laurence [2003]. According to LDA measurements, the flow in the tube bank becomes periodic at about the fourth row; the data are for the $4^{\text {th }}-6^{\text {th }}$ rows . Periodic conditions indicate that the inflow at the left is equal to the outflow to the right. Also, the flow at the top is equal to the flow at the bottom. Hassan and Barsamian [2004], however, use the full depth of the tube bank (for the same data) because they believe that the largest turbulent length scale of the flow is large enough to require it. The mass flow is set at a given value to provide a flow through the flow domain. Although the mean velocity upstream of the tube bundle is reported to be $1.06 \mathrm{~m} / \mathrm{sec}$, the data reported for the mean streamwise velocity do not correspond to this velocity. A B-spline curve was fitted to the mean streamwise velocity data and numerically integrated using the midpoint rule on a fine grid. The data reported by Simonin and Barcouda show a zero value for $U$ at $(x, y)=(0,0.012 \mathrm{~m})$. Actually, the radius

\footnotetext{
${ }^{\mathrm{b}}$ see ERCOFTAC database: http://cfd.me.umist.ac.uk/ercoftac/
} 
of the tube is $0.01085 \mathrm{~m}$. The B-spline curve is set to zero at $0.01085 \mathrm{~m}$, with a sharp increase, which corresponds to the direct numerical simulations (DNS) of the same flow shown by Benhamadouche and Laurence [2003]. The mass flow used for the periodic boundary conditions in the streamwise direction is based on the mass flow computed from the numerical integration of the B-spline curve. This value is $40.75 \mathrm{~kg} / \mathrm{sec}$, based on a water density of $998.2 \mathrm{~kg} / \mathrm{m}^{3}$, an area of $(0.045-.0217) \mathrm{m}$, and an average velocity of $1.752 \mathrm{~m} / \mathrm{sec}$. The Reynolds number based on the computed mass flow and the tube diameter is about 23,000, much less than for the flow in the VHTR lower plenum $\left(\operatorname{Re}>10^{5}\right)$.

\subsection{Steady State Calculations of Flow in Tube Bundles}

Benhamadouche and Laurence [2003] discuss the results of a number of workers who have simulated flow in tube banks and either assumed a stationary flow or used an unsteady approach that converged to steady state. Benhamadouche and Laurence use an unsteady approach, which does not converge to a steady state, claiming that a superior numerical scheme with lower numerical diffusion prevented convergence to a steady state. Results for several turbulence models assuming steady state flow conditions were shown and compared in Johnson [2005]. This section reviews the important findings of that study for continuity.

The Statement of Numerical Accuracy ${ }^{\mathrm{c}}$, published by the American Society of Mechanical Engineers (ASME) Journal of Fluids Engineering [1996] for papers submitted for publication in that journal, is used as a guide for both steady and unsteady numerical simulations performed in this study. These guidelines are:

1. The basic features of the method, including formal truncation error of individual terms in the governing numerical equations, must be described.

2. Methods must be at least second order accurate in space.

3. Inherent or artificial viscosity (or diffusivity) must be assessed and minimized.

4. Grid independence or convergence must be established.

5. When appropriate, iterative convergence must be addressed.

6. In transient calculations, phase error must be assessed and minimized.

7. The accuracy and implementation of boundary and initial conditions must be fully explained.

8. An existing code must be fully cited in easily available references.

9. Benchmark solutions may be used for validation for a specific class of problems.

10. Reliable experimental results may be used to validate a solution.

FLUENT $^{\circledR}$ is employed using 2 D double precision running on a 12 -cpu Sun 4800 workstation. FLUENT $^{\circledR}$ uses the finite volume method. The discretization of the terms is given in the FLUENT ${ }^{\circledR}$ Users' Manual. The viscous terms are discretized using a centered difference scheme ( $2^{\text {nd }}$ order), and the pressure term is evaluated using a centered scheme. The discretization scheme for the convective terms is specified by the user. The $2^{\text {nd }}$ order Quadratic Upstream Interpolation for Convective Kinematics (QUICK) scheme [Johnson and MacKinnon, 1992] is used herein. There is no added, only inherent, numerical viscosity present in the discretization scheme. This error is minimized by using a fine enough mesh. The segregated solver is used to solve the discretized equations; the default under-relaxation factors are used. The SIMPLE algorithm is used for pressure-velocity coupling.

\footnotetext{
${ }^{\mathrm{c}}$ http://journaltool.asme.org/Templates/JFENumAccuracy.pdf
} 
Finite volume grids of various finenesses were created for the flow problem. After grid independence tests were performed, it was concluded that the grid illustrated in Figure 4a (14,976 cells) was sufficiently fine to yield grid-independent results. The FLUENT ${ }^{\circledR}$ manual recommends that the dimensionless distance from the wall $\mathrm{y}^{+}\left(\equiv \mathrm{u}_{\tau} \mathrm{y} / \mathrm{v}, \mathrm{u}_{\tau}=\left[\tau_{\mathrm{w}} / \rho\right]^{1 / 2}\right.$, where $\tau_{\mathrm{w}}$ is the wall shear stress $)$ to the middle of the first cell be greater than about 30 . Figure $4 \mathrm{~b}$ plots the $\mathrm{y}^{+}$values for all of the five walls in the flow domain for the grid. The quarter tube walls are labeled as 'wall1' to 'wall4' starting in the lower left corner of the grid and moving clockwise. Wall 'wallcen' is the center tube. The wall functions are designed to be useable for all ranges of $y^{+}$. Though the FLUENT ${ }^{\circledR}$ manual recommends that $\mathrm{y}^{+}$values for standard wall functions be greater than a value of about 30, it was found that the grid of Figure $4 \mathrm{a}$ with $\mathrm{y}^{+}$ $\leq 5$, shown in Figure 4b, provided the best results for the RSM [Johnson, 2005].

An iterative convergence study was performed using the RSM with standard wall functions in FLUENT $^{\circledR}$. The solution (scaled) tolerance was set to values of $10^{-3}, 10^{-4}, 10^{-5}$ and $10^{-6}$ for every equation computed. Figure 5 compares the computed solutions against the Simonin and Barcouda data for the mean streamwise velocity $U$ and the normal Reynolds stress $\overline{v^{2}}$ for the four tolerances. As shown, the tolerances of $10^{-3}$ and $10^{-4}$ are not converged, even though the results for the tolerance of $10^{-4}$ provide very good agreement with the data for $U$ and $\overline{v^{2}}$. The results for $10^{-5}$ to $10^{-6}$ are very close, suggesting that iterative convergence should be required to $10^{-5}$ or less.

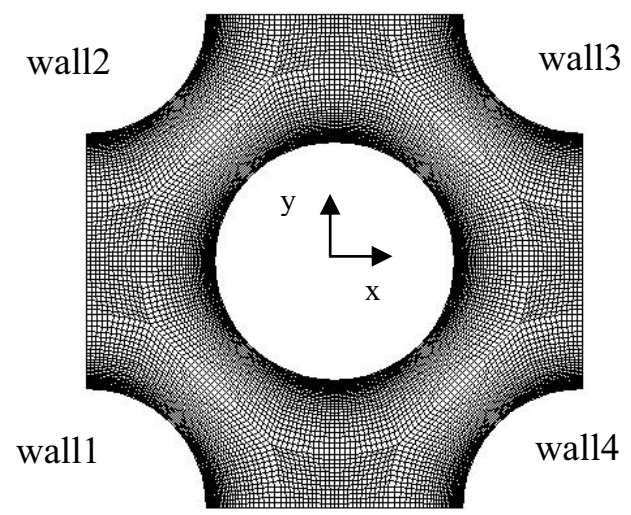

(a)

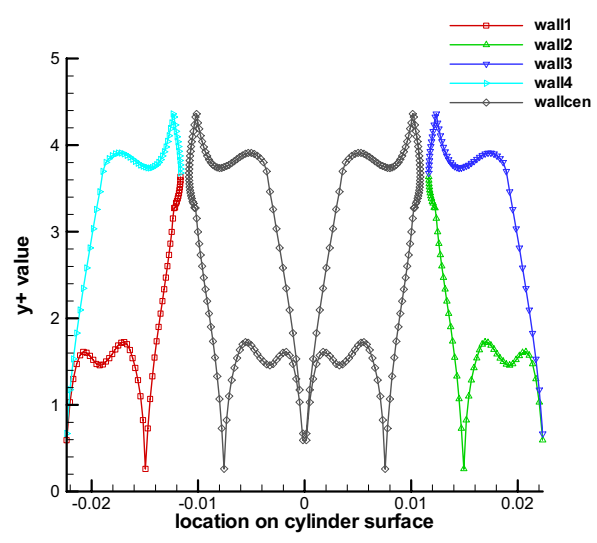

(b)

Figure 4. FLUENT ${ }^{\circledR}$ grid for the tube bundle simulations (a) and $\mathrm{y}^{+}\left(\equiv \mathrm{u}_{\tau} \mathrm{y} / \mathrm{v}\right)$ distribution for the grid (b). 


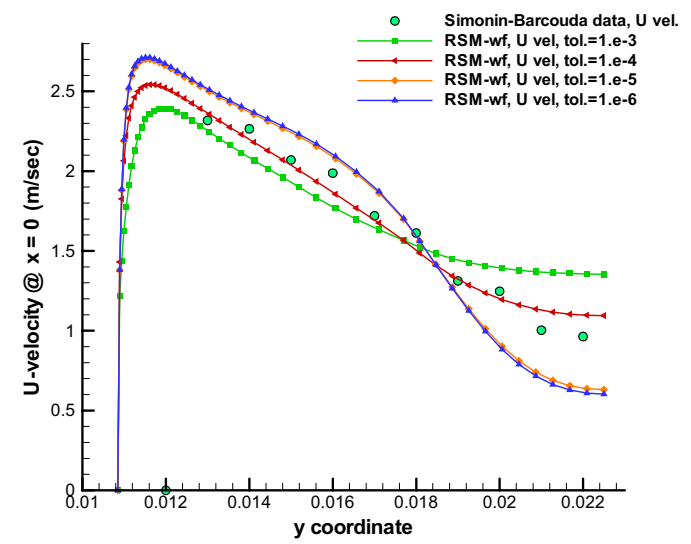

(a)

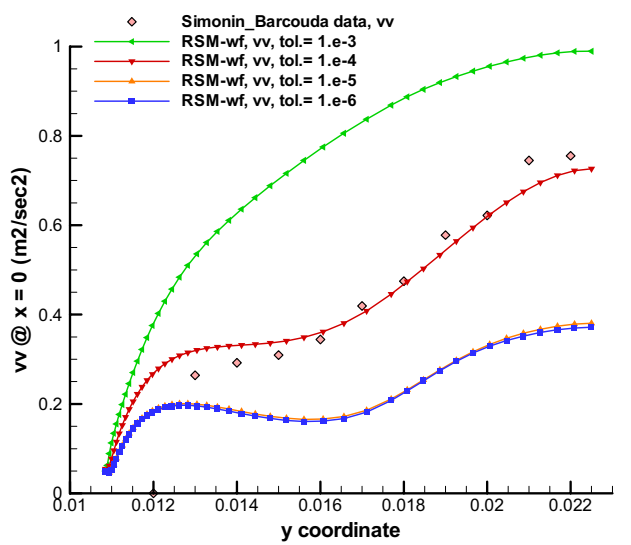

(b)

Figure 5. Comparison of solutions for iterative tolerances of $1 \times 10^{-3}$ to $1 \times 10^{-6}$ for (a) the mean streamwise velocity $U$ and (b) the normal stress $\overline{v^{2}}$ at $\mathrm{x}=0$.

Figure 6 illustrates the B-spline curve fitted to the Simonin and Barcouda data $U$ as well as comparisons to the results for the $\mathrm{k} \sim \varepsilon$ model using three different wall treatments: standard and nonequilibrium wall functions and the enhanced wall treatment. DNS and large eddy simulation (LES) calculations reported in Benhamadouche and Laurence [2003] indicate that there should be a sharp peak in the value of $U$ near the cylinder wall $(\mathrm{y}=0.01085 \mathrm{~m})$, whereas the eddy viscosity $\mathrm{k} \sim \varepsilon$ model results show rounded peaks that fall below the data.

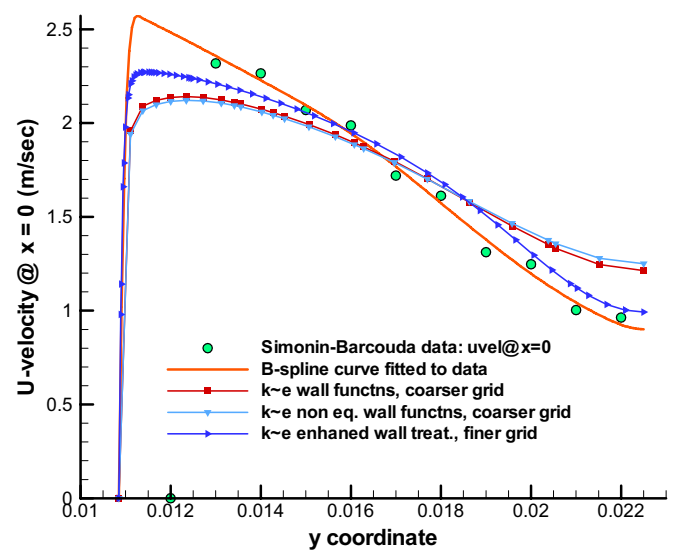

Figure 6. The B-spline fitted to the data of Simonin and Barcouda for $U$ at $\mathrm{x}=0$ compared to results for the $\mathrm{k} \sim \varepsilon$ model for standard and non-equilibrium wall functions and the enhanced wall treatment.

Calculations were made using the RSM for steady flow in Johnson [2005]. These are compared to calculations using the $\mathrm{v}^{2}-f$ model of Durbin [FLUENT ${ }^{\circledR}, 2005$ ] and the realizable $\mathrm{k} \sim \varepsilon$ model with enhanced wall treatment at $x=0$ in Figure 7 . The $\mathrm{v}^{2}-\mathrm{f}$ model solves an additional transport equation for $\overline{v^{2}}$, even though the value of $\overline{v^{2}}$ is given by the Boussinesq model, Eq. (8). However, $\overline{v^{2}}$ is used as the velocity scale in the specification of the turbulent viscosity. Hence, there are two distributions obtained for $\overline{v^{2}}$, one from the solution of the additional transport equation for $\overline{v^{2}}$, and one from the Boussinesq model. Clearly, the RSM results for $U$ show a sharp peak near the cylinder wall, which accords with the 
DNS and LES results reported in Benhamadouche and Laurence [2003]. The eddy viscosity results are rounded and fall below the Simonin and Barcouda data.

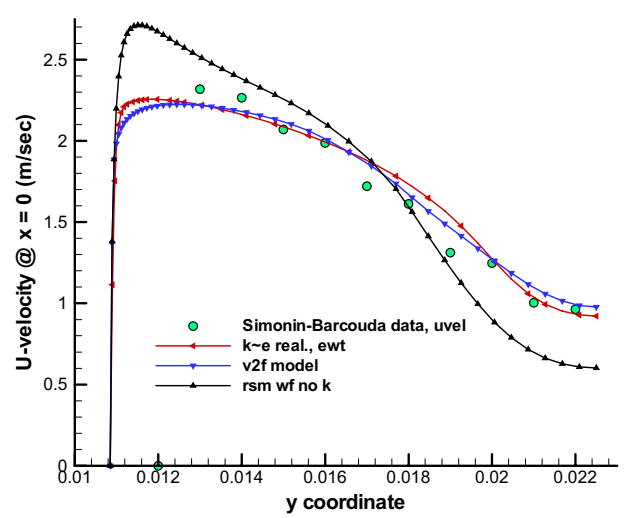

(a)

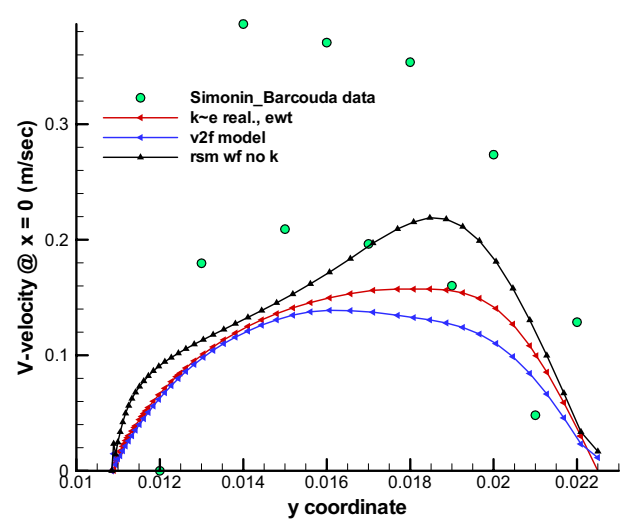

(b)

Figure 7. Mean velocity profiles at $\mathrm{x}=0$ for realizable $\mathrm{k} \sim \varepsilon, \mathrm{v}^{2}-\mathrm{f}$ and RSM models.

Figure 8 shows profiles for the three Reynolds stresses for the realizable $\mathrm{k} \sim \varepsilon$ model using the enhanced wall treatment, the $\mathrm{v}^{2}-\mathrm{f}$ model, and the RSM using wall functions. From Figure $8 \mathrm{~b}$, we see that the profile based on the transport equation for $\overline{v^{2}}$ does not match the profile computed by the Boussinesq model. We also see that the $\mathrm{v}^{2}$-f model is overall poorer than the realizable model, which, in turn, is poorer that the RSM in matching the data. For these reasons, including the better trends shown by the RSM results, it was determined that the RSM would be employed for the unsteady calculations reported in the following section. 


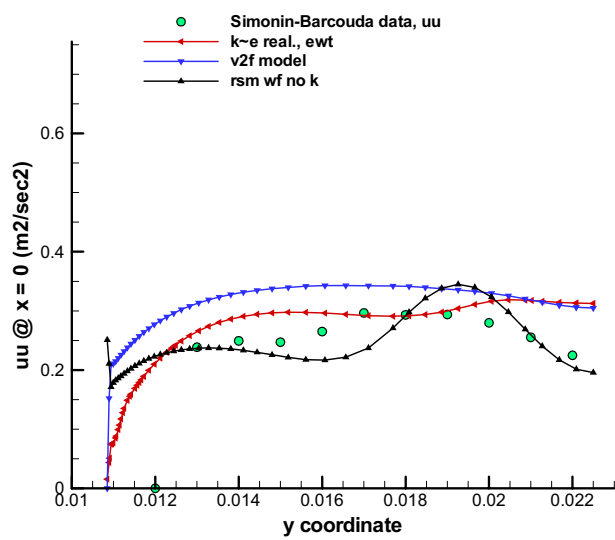

(a)

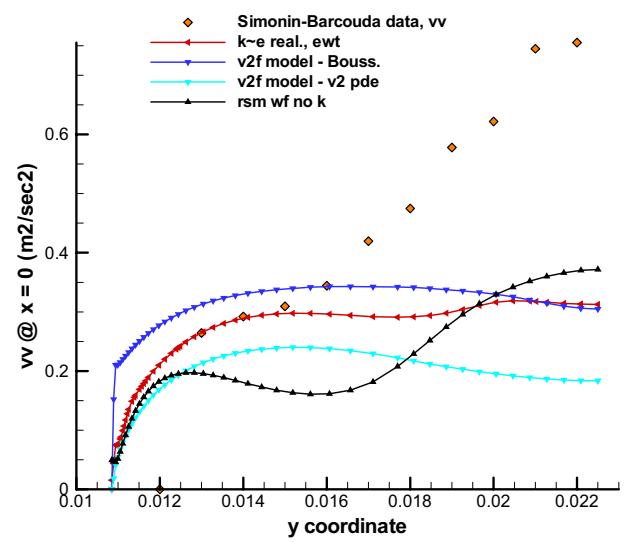

(b)

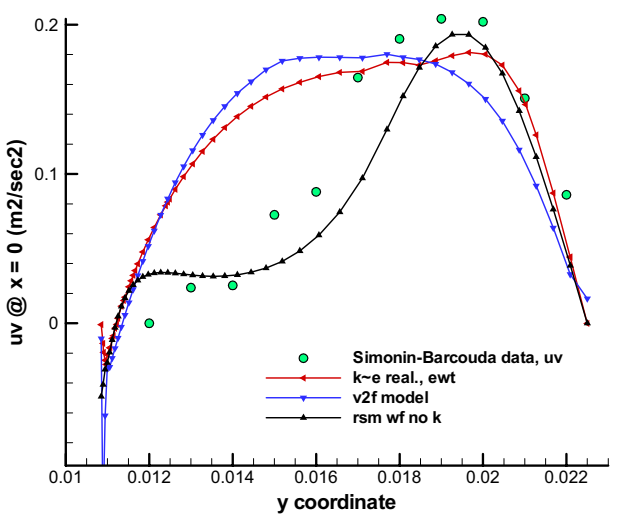

(c)

Figure 8. Results for (a) $\overline{u^{2}}$ and (b) $\overline{v^{2}}$ normal stresses and (c) the shear stress $\overline{u v}$ for the realizable $\mathrm{k} \sim \varepsilon$ model using the enhanced wall treatment, the $\mathrm{v}^{2}-\mathrm{f}$ model, and the RSM using wall functions at $\mathrm{x}=0$.

\subsection{Unsteady Calculations of Flow in Tube Bundles}

Hassan and Barsamian [2004] indicate that the flow in staggered tube bundles reported by Simonin and Barcouda [1986, 1988] is a non-stationary turbulent flow. Benhamadouche and Laurence [2003] also compute the flow as a non-stationary turbulent flow. That is, the flow is reported to have non-turbulent fluctuations; these fluctuations are associated with vortex-shedding from the tubes. The Reynoldsaveraging of the fluid flow (Navier-Stokes) equations is designed to separate the mean velocity from the turbulent fluctuations, which are then modeled with a so-called RANS turbulence model. However, turbulence models are not designed to model the effects of coherent fluctuations. This is in contrast to LES and DNS, which compute directly all fluctuations that are larger than the filter/grid. A RANS turbulence model can be used in an unsteady calculation for a non-stationary turbulent flow. In this case, the correlation of coherent fluctuations can be computed directly. Such a calculation is denoted an unsteady RANS (URANS) calculation [Hanjalic, 2005].

A stationary turbulent flow in tube bundles (no coherent unsteadiness) is physically different from a non-stationary turbulent flow. In the first case, a simple time-averaging is adequate to separate the mean velocity and the turbulent fluctuations. A turbulence model applied to such a flow is called a RANS calculation. In the second case, the flow can be ensemble averaged. Theoretically, an ensemble 
average is applied to experimental data by running the experiment a large number of times and taking data at selected points and at selected times. The data for a given point at a specified time from each of the tests are averaged to obtain the ensemble average. The ensemble average may still be unsteady due to non-turbulent fluctuations. The difference between the ensemble average and the instantaneous velocity are the turbulent fluctuations. If the flow is assumed to be unsteady and modeled as such, the RANS turbulent model is used to model the turbulent fluctuations. The Reynolds stresses thus obtained still vary in time as does the ensemble average velocity. The ensemble-averaged quantities may be further averaged in time to remove all unsteadiness. FLUENT ${ }^{\circledR}$ [2005] allows this type of calculation in postprocessing and refers to it as the 'mean velocity.' The mean velocity is obtained by averaging the velocity at a point over a period of time. If the flow exhibits both turbulent and non-turbulent unsteadiness, then this averaging can be seen as the time average of the ensemble average or just a long-time average.

If the coherent unsteadiness is a well-behaved periodic unsteadiness, the flow may be phaseaveraged, where data are taken at the same time in each cycle to obtain a collection of data that can then be averaged to obtain a mean and turbulent fluctuating quantity. Thus, phase averaging is a special case of ensemble averaging. In the case of the latter, the ensemble mean quantities must be repeatable from the initial condition, but not necessarily periodically. The ensemble-averaged equations of the flow and continuity equations are still referred to as being Reynolds-averaged. Prior to ensemble averaging, a Reynolds decomposition is accomplished for the dependent variables:

$$
u=\langle u\rangle+u^{\prime}
$$

where:

$u$ is the instantaneous quantity (velocity, pressure, temperature)

$\langle u\rangle$ is the ensemble mean quantity

$u^{\prime}$ is the turbulent fluctuation.

Figure 9 illustrates the concept of ensemble averaging with simple hand-drawn curves that show the relationships inherent in Eq. (10). After the dependent variables in the Navier-Stokes and continuity are decomposed according to Eq. (10), the equations are ensemble averaged (Reynolds-averaged) to obtain the equations (similar to Eqs. [5,6]):

$$
\begin{gathered}
\frac{\partial\left\langle u_{i}\right\rangle}{\partial x_{i}}=0 \\
\rho \frac{\partial\left\langle u_{i}\right\rangle}{\partial t}+\rho\left\langle u_{j}\right\rangle \frac{\partial\left\langle u_{i}\right\rangle}{\partial x_{j}}=-\frac{\partial\langle p\rangle}{\partial x_{j}}+\frac{\partial}{\partial x_{j}}\left[\mu\left(\frac{\partial\left\langle u_{i}\right\rangle}{\partial x_{j}}+\frac{\partial\left\langle u_{j}\right\rangle}{\partial x_{i}}\right)-\rho\left\langle u_{i}^{\prime} u_{j}^{\prime}\right\rangle+\rho f_{i}\right]
\end{gathered}
$$

where $\rho\left\langle u_{i}^{\prime} u_{j}^{\prime}\right\rangle$ is the (time-varying) Reynolds stress tensor. Equations $(11,12)$ are those that are solved for the present unsteady simulations. 


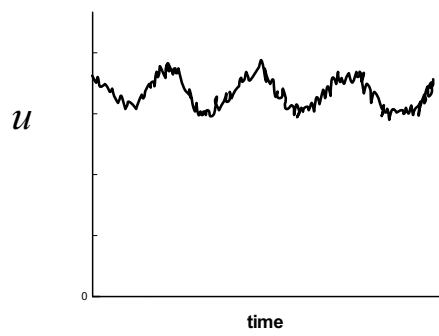

(a)

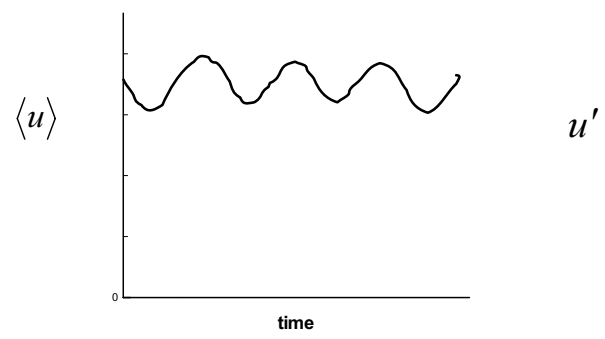

(b)

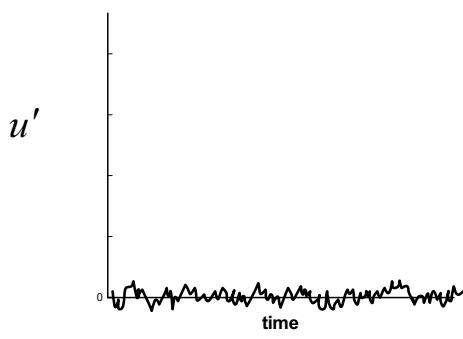

(c)

Figure 9. (a) The instantaneous quantity $u$ for a point in space and over a period of time, along with (b) the ensemble average $\langle u\rangle$ of the signal and (c) the (turbulent) fluctuating component $u^{\prime}$.

The ensemble-averaged quantities may additionally be time averaged (with a time interval long enough to adequately average all coherent fluctuations) with a secondary decomposition:

$$
\langle u\rangle=U+\tilde{u}
$$

where $U$ is the time-averaged quantity and $\tilde{u}$ is the coherent (non-turbulent) fluctuating quantity. Figure 10 illustrates the concept of the time- and ensemble-averaged non-turbulent fluctuating quantities.

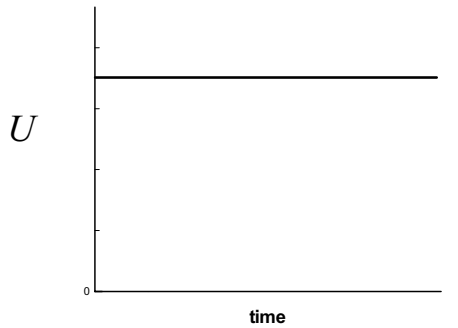

(a)

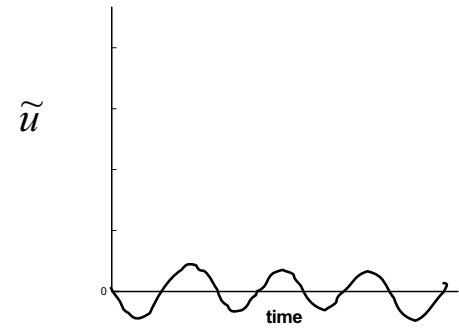

(b)

Figure 10. The (a) time- and ensemble- averaged quantity and (b) the non-turbulent fluctuating quantity.

The purpose for performing this second (time) averaging is that some data provided by experimentalists, such as Simonin and Barcouda [1986, 1988], may have been processed with a long-time averaging rather than ensemble-averaged data. By performing just a long-time averaging procedure, the turbulent fluctuations are combined with the coherent fluctuations such that only their sum is provided. Upon performing the second decomposition, Eq. (13), and then time-averaging the equations, one arrives at the following equations:

$$
\begin{gathered}
\frac{\partial U_{i}}{\partial x_{i}}=0 \\
\rho \frac{\partial U_{i}}{\partial t}+\rho U_{j} \frac{\partial U_{i}}{\partial x_{j}}=-\frac{\partial P}{\partial x_{j}}+\frac{\partial}{\partial x_{j}}\left[\mu\left(\frac{\partial U_{i}}{\partial x_{j}}+\frac{\partial U_{j}}{\partial x_{i}}\right)-\rho \overline{\widetilde{u}_{i} \widetilde{u}_{j}}-\rho \overline{\left\langle u_{i}^{\prime} u_{j}^{\prime}\right\rangle}\right]+\rho f_{i}
\end{gathered}
$$


where

$$
\begin{aligned}
& \rho \overline{\widetilde{u}_{i} \widetilde{u}_{j}} \text { is a time correlation of coherent fluctuations } \\
& \rho \overline{\left\langle u_{i}^{\prime} u_{j}^{\prime}\right\rangle} \text { is the time-average of the Reynolds stress tensor. }
\end{aligned}
$$

If we had performed a long time average to the instantaneous equations such that all fluctuations were captured, we would have obtained Eqs. (5) and (6), except that the correlation term would include both coherent and turbulent fluctuations. Denoting the velocity fluctuation, whether turbulent or coherent by $u_{i}^{\prime \prime}$, the long time-averaged Navier Stokes equations can be written:

$$
\rho \frac{\partial U_{i}}{\partial t}+\rho U_{j} \frac{\partial U_{i}}{\partial x_{j}}=-\frac{\partial P}{\partial x_{j}}+\frac{\partial}{\partial x_{j}}\left[\mu\left(\frac{\partial U_{i}}{\partial x_{j}}+\frac{\partial U_{j}}{\partial x_{i}}\right)-\rho \overline{u_{i}^{\prime \prime} u_{j}^{\prime \prime}}\right]+\rho f_{i}
$$

Comparing Eq. (15) with Eq. (16) we can write:

$$
\rho \overline{u_{i}^{\prime \prime} u_{j}^{\prime \prime}}=\rho \overline{\widetilde{u}_{i} \widetilde{u}_{j}}+\rho \overline{\left\langle u_{i}^{\prime} u_{j}^{\prime}\right\rangle}
$$

We see that the total correlation obtained incorporating all fluctuations is equivalent to the sum of two separate correlations, one for the coherent fluctuations and one for the turbulent fluctuations. Eq. (16) is, in fact, the time-averaged Navier-Stokes equation that is normally obtained for a stationary turbulent flow or in the event that one mistakenly assumes that the flow is stationary.

An unsteady FLUENT ${ }^{\circledR}$ calculation can be made of the flow of Simonin and Barcouda. The FLUENT $^{\circledR}$ post-processor provides the ensemble- and time-averaged mean quantities (or just long-time averaged) as well as RMS values of the fluctuations. If a RANS turbulence model is used to represent the effects of turbulence, the fluctuations will be the non-turbulent (coherent) fluctuations. These values must be squared to obtain the coherent term in Eq. (6). To obtain the time-average of the Reynolds stresses, a User-Defined function (UDF) must be written. Also, coherent cross-correlations $(i \neq j)$ must be obtained using a UDF because FLUENT ${ }^{\circledR}$ does not provide these. The coherent term can then be added to the turbulence term to obtain the total fluctuating correlation to be compared with the Simonin and Barcouda data. To obtain the cross-correlation for the coherent fluctuations, we can write:

$$
\overline{\langle u\rangle\langle v\rangle}=\overline{(U+\widetilde{u})(V+\widetilde{v})}=\overline{U V+\widetilde{v} U+\widetilde{u} V+\widetilde{u} \widetilde{v}}=U V+\overline{\widetilde{u}}
$$

since the time average of a coherent fluctuating component is zero. From Eq. (16), the fluctuating correlation can be obtained by subtracting the product $U V$ (mean quantities) from the time-average of the product of the ensemble-averaged velocities (computed in the UDF). The UDF is provided in the Appendix.

For the URANS calculations, the second-order implicit time differencing scheme is employed. The time step was set to be adaptive with the minimum time step set to $10^{-5}$ seconds and the maximum set to 5 $\times 10^{-3}$. The time step size was usually between 2 and $5 \times 10^{-4} ; 5000$ time steps were computed for the present results. The results were compared with varying numbers of time steps to ensure that the timeaveraged results are unchanging. A time step required about 22 seconds on the Sun 4800 . The default truncation error tolerance of 0.01 for the time step was used. Figure 11 illustrates the ensemble-averaged 
streamwise velocity $\langle u\rangle$ at two points in the flow as functions of time step. The time step is clearly small enough to capture the large scale motions without unphysical oscillations.

It was found that if the residual tolerance was set to $10^{-5}$ or $10^{-6}$ and enough iterations at each time step allowed for convergence (1000), the simulation remained steady state. However, when the maximum number of iterations was set to 110 for each time step, with the residual tolerance set to $10^{-6}$, it was found that the solution would converge every other time step or occasionally every third time step to the desired tolerance, while it would still converge to $1.5 \times 10^{-5}$ or lower on the other time steps. This approach apparently allowed there to be vortex shedding, due most likely to a numerical 'kick' caused by the uneven convergence, though the convergence is adequate according to the iterative convergence test described earlier. It is not unusual that a numerical perturbation is required to initiate unsteadiness in a simulation that is, in reality, an unsteady flow. Hence, it is believed that the above approach used for the unsteady flow calculation is reasonable and acceptable. Furthermore, the results for the unsteady calculations will be shown below to better match the Simonin and Barcouda data.

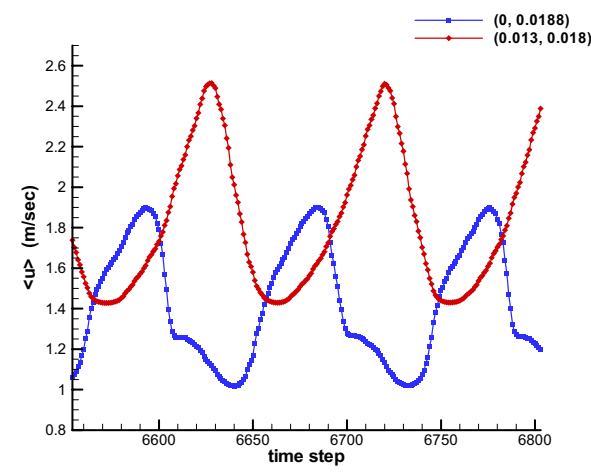

Figure 11. Time plots of the ensemble average velocity at two points in the flow for 250 steps.

The Simonin and Barcouda data include results for the mean streamwise and transverse velocities, $U$ and $V$; the two normal stresses, $\overline{u^{\prime \prime 2}}$ and $\overline{v^{\prime \prime 2}}$; and the shear stress $\overline{u^{\prime \prime} v^{\prime \prime}}$ profiles at five locations. Comparisons are made at five locations: $\mathrm{x}=0,11 \mathrm{~mm}$, and $16.5 \mathrm{~mm}$ and $\mathrm{y}=0$ and $22.5 \mathrm{~mm}$. The Simonin and Barcouda data for the Reynolds stresses are given as constant and must, therefore ,have been (long) time-averaged, such that they include the non-turbulent fluctuating components.

Figures 12-16 illustrate the computed results for the mean and fluctuating quantities at the above locations for the steady RANS and URANS approaches compared to the data of Simonin and Barcouda. In the case of the URANS results, the long-time averaged or time-averaged ensemble-averaged results are plotted. For the fluctuating quantities, the contributions for both the coherent and turbulent fluctuations are each shown along with their sums. It can be seen that the results for URANS for the mean velocity $U$ are very close to the data for $\mathrm{x}=0,11$, and 16.5 and close for $\mathrm{y}=0$. The results for steady RANS are clearly inferior to the URANS results compared to the Simonin and Barcouda data. The present 2D URANS RSM results for $U$ are comparable to 3D URANS RSM results of Benhamadouche and Laurence [2003], except that the present results are superior for $U$ at $y=0$. The RSM of Benhamadouche and Laurence is somewhat different from the present one in that the Benhamadouche and Laurence version does not use the pressure reflection term, nor does it employ the eddy viscosity in the dissipation rate equation as does FLUENT ${ }^{\circledR}$. Also, the grid of Benhamadouche and Laurence is slightly coarser. Benhamadouche and Laurence claim that $3 \mathrm{D}$ calculations are necessary and give $2 \mathrm{D}$ results that are quite inferior to their 3D results. 
The mean velocity $V$ data at $\mathrm{x}=0$ are actually two profiles, one taken above the center cylinder and one below [Laurence, 2005], then drawn mirrored. The differences in the two profiles is an indication of the uncertainty in the measurements at this location. The present 2D computations are quite close to the far more costly 3D URANS, LES, and DNS calculations reported in Benhamadouche and Laurence. The present results for $V$ at $\mathrm{x}=11 \mathrm{~mm}$ and $16.5 \mathrm{~mm}$ are close to the Simonin and Barcouda data for both the RANS and URANS approaches. At y $=0,22.5 \mathrm{~mm}$, symmetry arguments require $V$ to be zero; the calculations are quite close to zero.

For the normal stress $\overline{u^{\prime \prime}}$, the URANS results are poorer than the RANS results and show large departures from the Simonin and Barcouda data. It is also the case that the computed results of Benhamadouche and Laurence for $\overline{u^{\prime \prime}}$ show significantly better agreement with Simonin and Barcouda than the present results. However, for $\overline{v^{\prime \prime 2}}$, the present URANS calculations are all significantly better than for the earlier RANS calculations, though the magnitude of the maximum in each case is not as high as the Simonin and Barcouda data. The importance of the contribution of the coherent fluctuations is clearly shown in the figures. The present results for the normal stresses are not as good as for Benhamadouche and Laurence's 3D results, but range from the same to far better than the 2D Benhamadouche and Laurence results. Finally, results for the shear stress $\overline{u^{\prime \prime} v^{\prime \prime}}$ are better than or about the same for both unsteady and steady results and agree quite well with the Simonin and Barcouda data; the shear stress should be zero for $\mathrm{y}=0$ and $22.5 \mathrm{~mm}$ based on symmetry. The most important fluctuating stress is the shear stress because it represents transport of momentum in an orthogonal direction from the momentum equation in question, whereas the normal stresses could actually be combined with the pressure term to represent a modified pressure. (The normal stresses appear with a gradient in the same direction as the pressure.) 


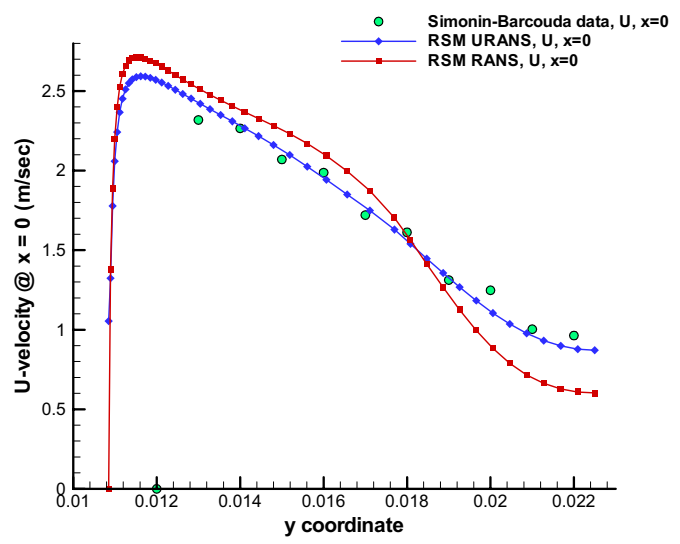

(a)

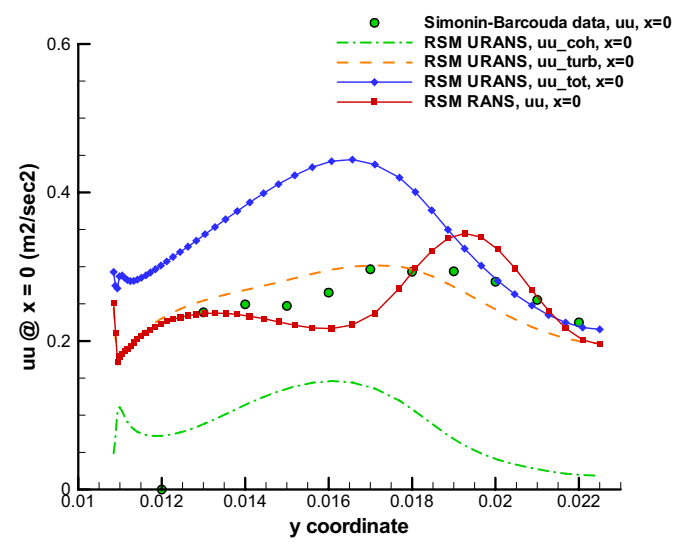

(c)

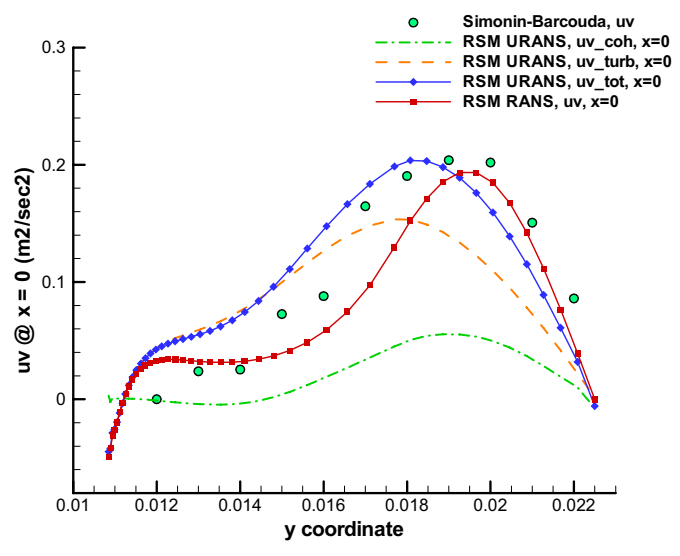

(e)

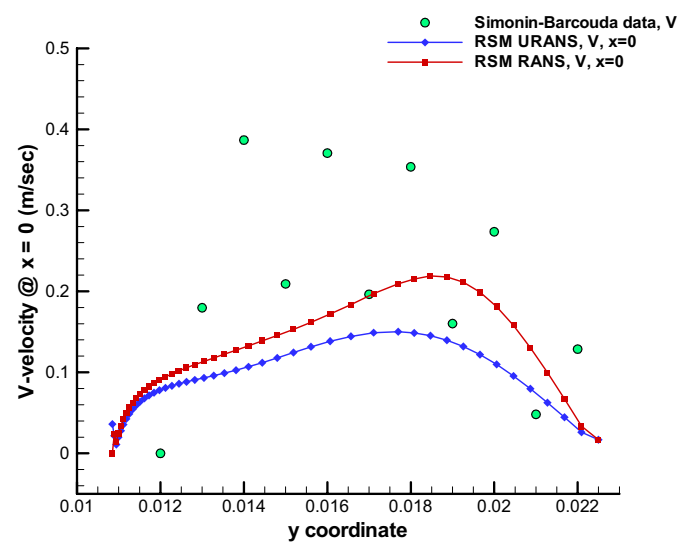

(b)

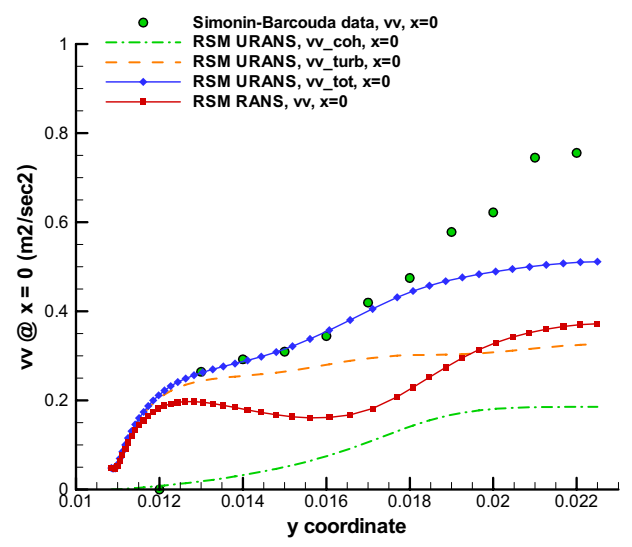

(d)

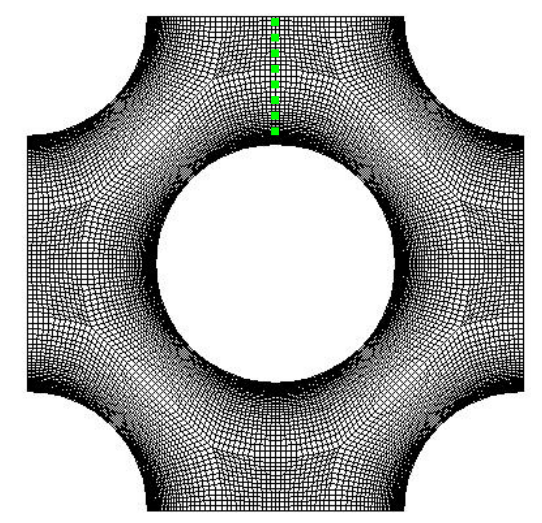

(f)

Figure 12. URANS results compared to steady RANS results for $x=0$ for (a) the mean streamwise velocity $U$, (b) the mean transverse velocity $V$, and the time averaged Reynolds stresses, including the coherent and turbulent correlations and their total: (c) the streamwise normal, (d) the transverse normal and (e) the cross-correlation, plus (f) the profile location superimposed on the finer grid. 


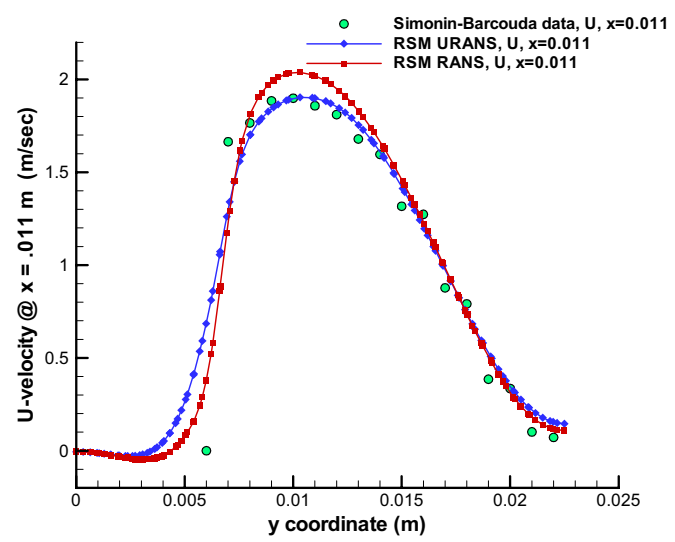

(a)

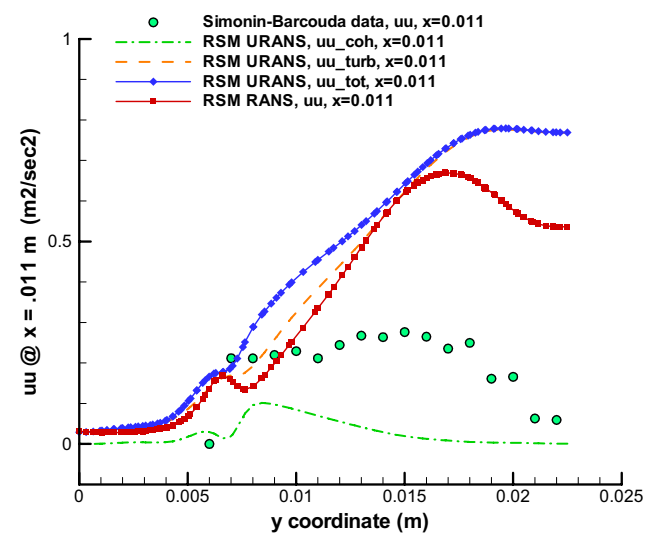

(c)

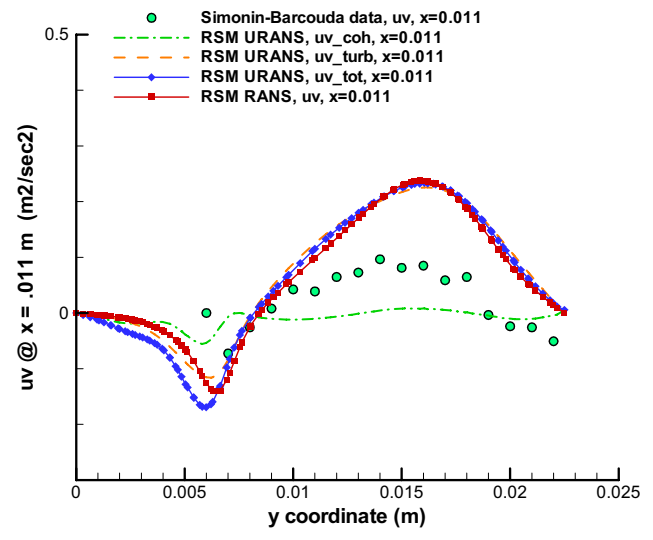

(e)

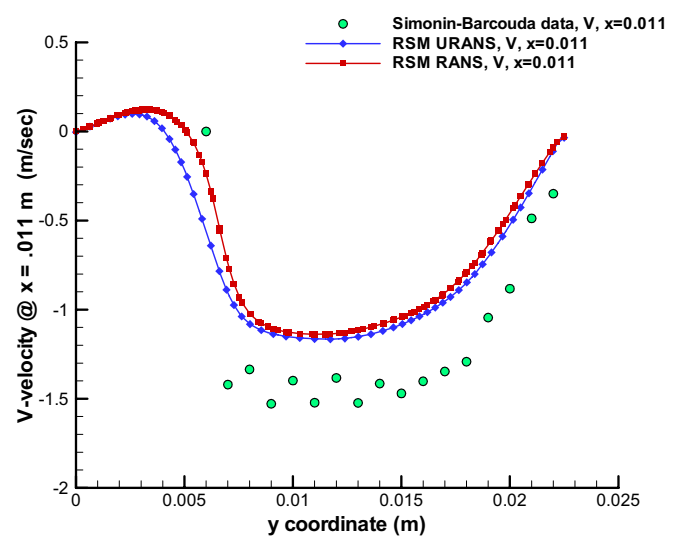

(b)

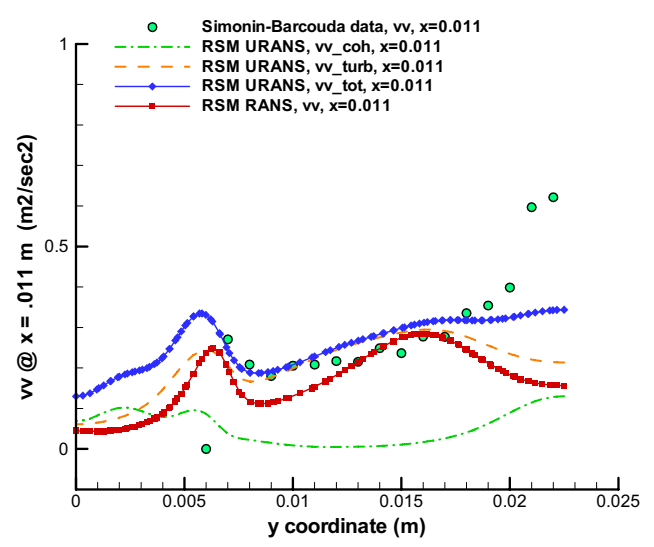

(d)

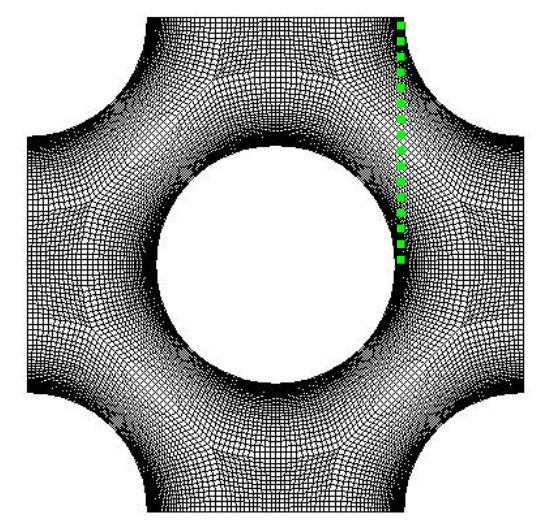

(f)

Figure 13. URANS results compared to steady RANS results for $x=11 \mathrm{~mm}$ for (a) the mean streamwise velocity $U$, (b) the mean transverse velocity $V$, and the time averaged Reynolds stresses, including the coherent and turbulent correlations and their total: (c) the streamwise normal, (d) the transverse normal and (e) the cross-correlation, plus (f) the profile location superimposed on the finer grid. 


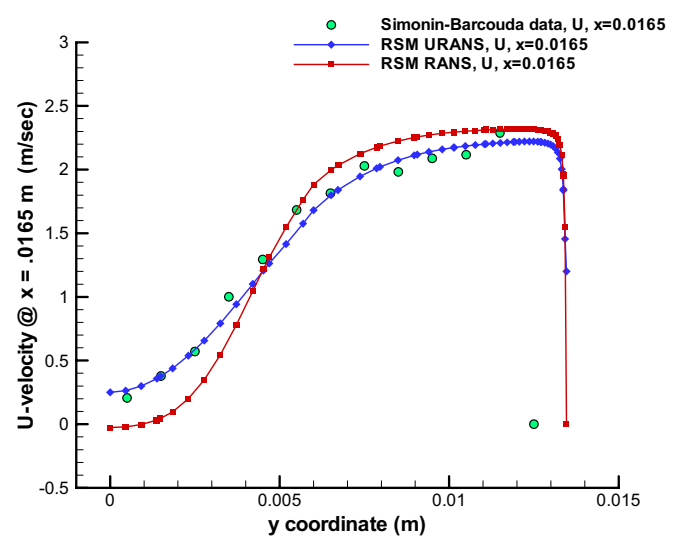

(a)

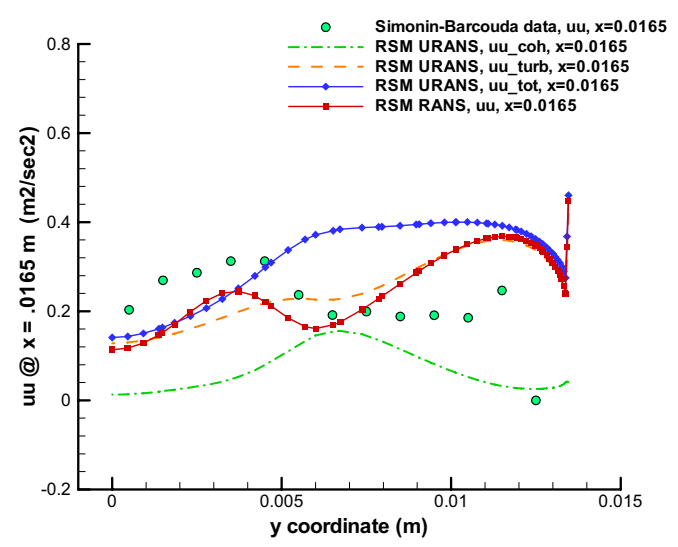

(c)

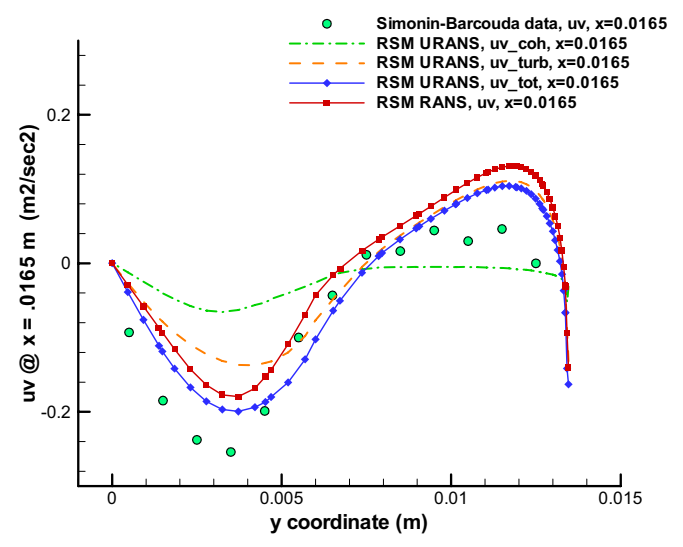

(e)

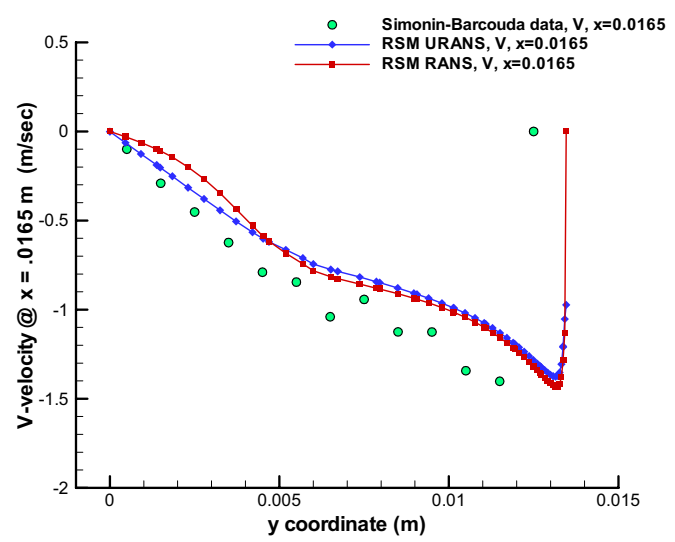

(b)

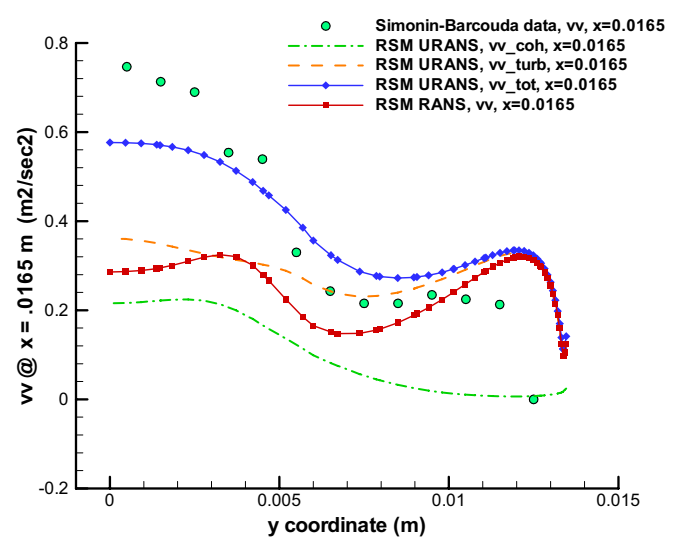

(d)

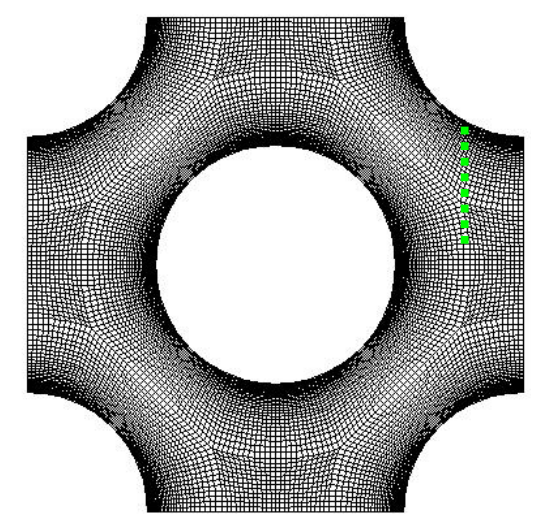

(f)

Figure 14. URANS results compared to steady RANS results for $x=16.5 \mathrm{~mm}$ for (a) the mean streamwise velocity $U$, (b) the mean transverse velocity $V$, and the time averaged Reynolds stresses, including the coherent and turbulent correlations and their total: (c) the streamwise normal, (d) the transverse normal and (e) the cross-correlation, plus (f) the profile location superimposed on the finer grid. 


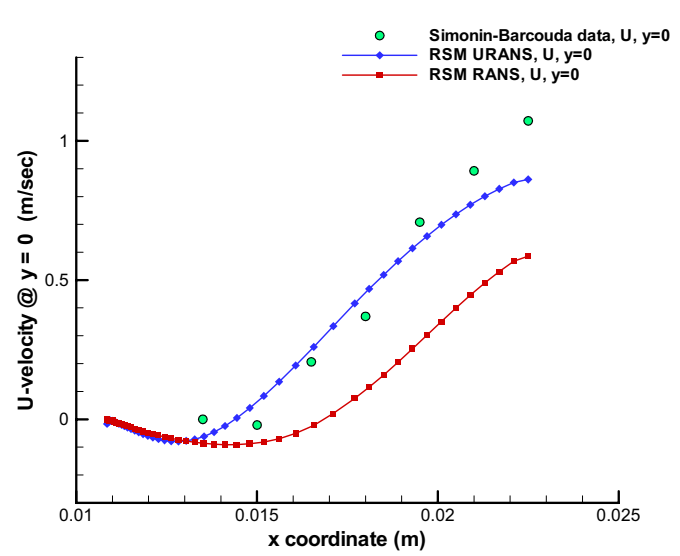

(a)

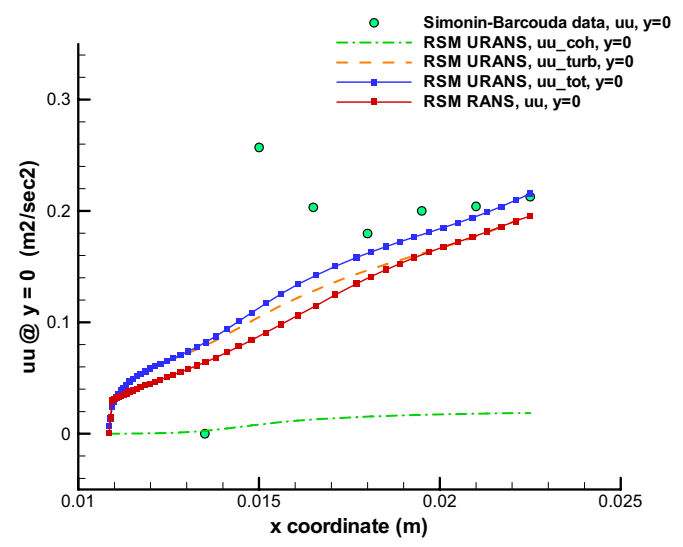

(c)

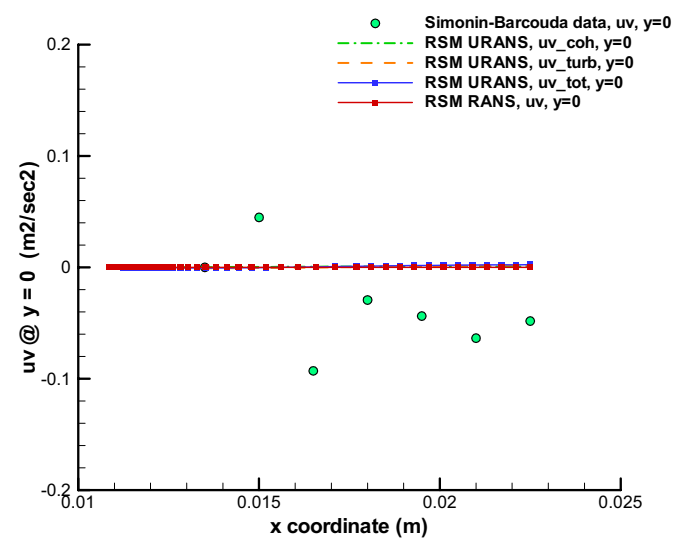

(e)

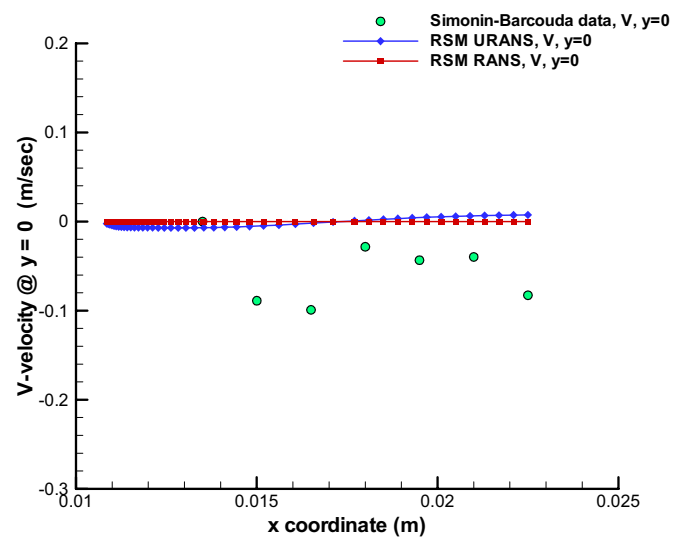

(b)

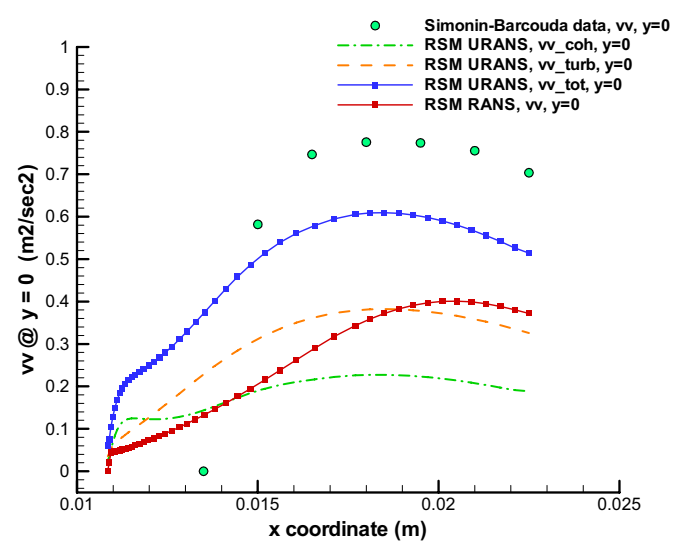

(d)

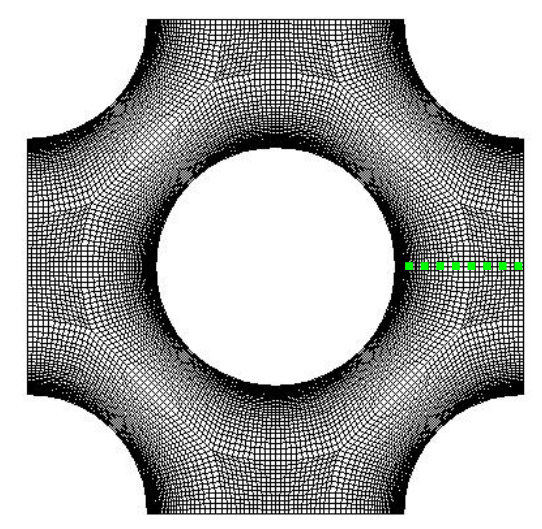

(f)

Figure 15. URANS results compared to steady RANS results for $y=0$ for (a) the mean streamwise velocity $U$, (b) the mean transverse velocity $V$, and the time averaged Reynolds stresses, including the coherent and turbulent correlations and their total: (c) the streamwise normal, (d) the transverse normal and (e) the cross-correlation, plus (f) the profile location superimposed on the finer grid. 


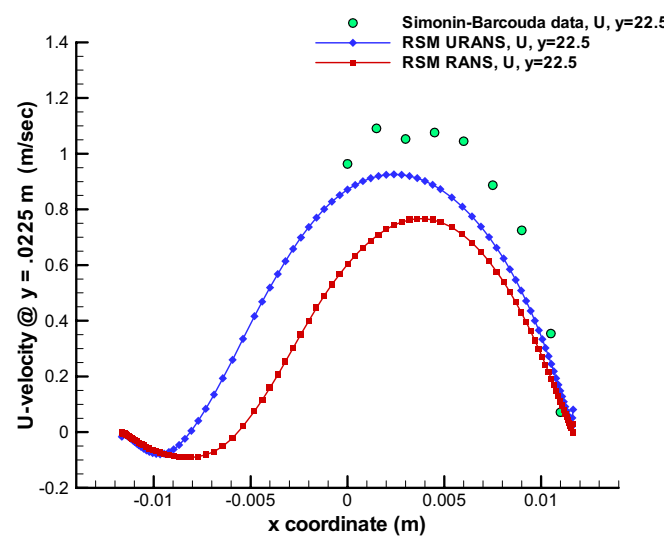

(a)

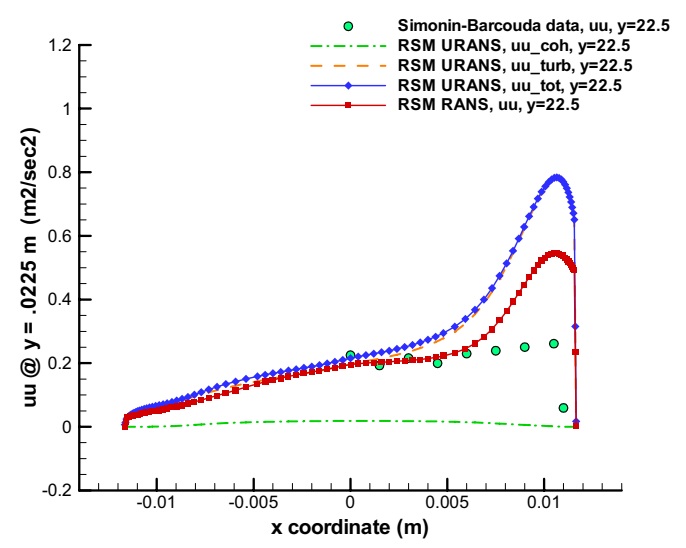

(c)

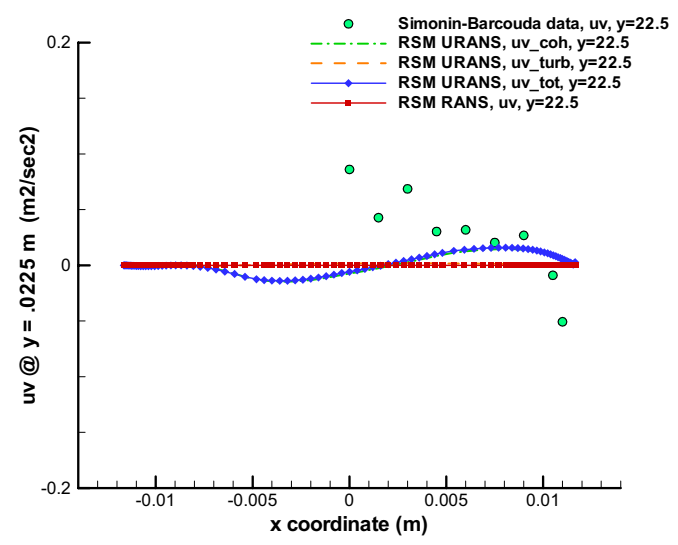

(e)

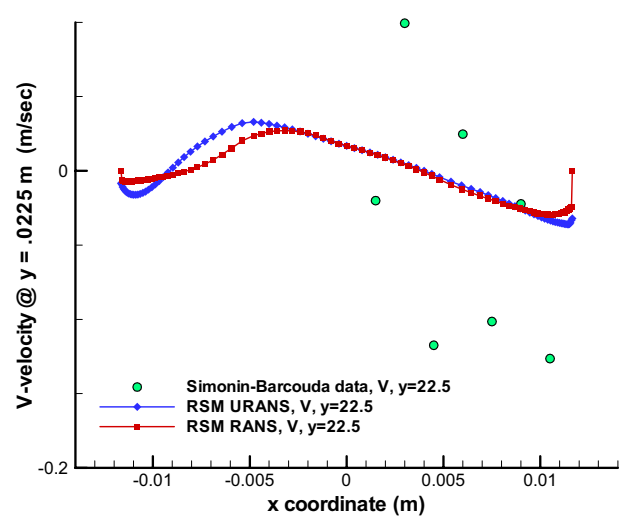

(b)

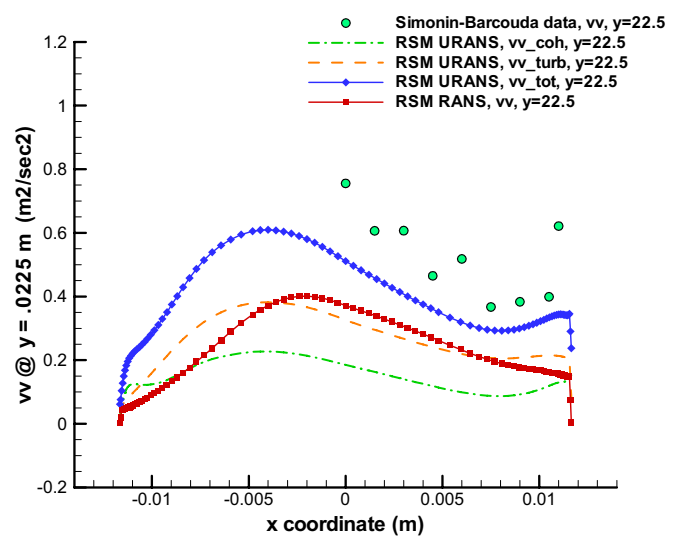

(d)

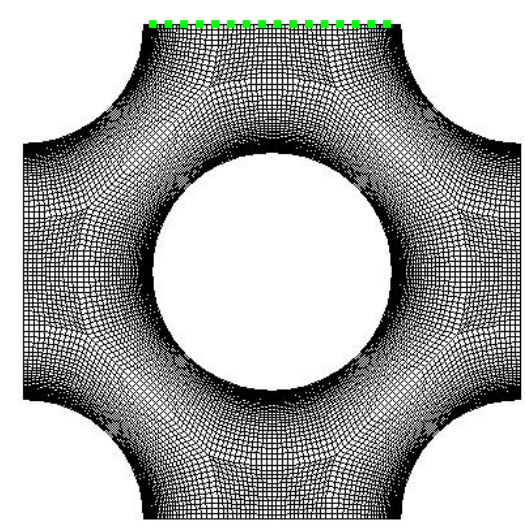

(f)

Figure 16. URANS results compared to steady RANS results for $y=22.5 \mathrm{~mm}$ for (a) the mean streamwise velocity $U$, (b) the mean transverse velocity $V$, and the time averaged Reynolds stresses, including the coherent and turbulent correlations and their total: (c) the streamwise normal, (d) the transverse normal and (e) the cross-correlation, plus (f) the profile location superimposed on the finer grid. 


\subsection{Conclusions}

The present 2D URANS results for the (long-time averaged) mean velocities for the tube-bank flow are quite satisfactory relative to the Simonin and Barcouda data, especially the streamwise velocity, and show correct trends. A primary reason for this is that the flow has been correctly computed as a nonstationary flow. Previous computations, which examined various turbulence models, assumed a steady flow. The shear Reynolds stress results are also satisfactory and show correct trends. While predictions for the normal stresses are not always satisfactory, they appear to have a relatively small effect on the results for the mean velocities. However, it is not yet clear how important accurate predictions for the Reynolds stresses are to obtaining accurate results for the mixing of the hot and cooler jets in the lower plenum.

Recommendations for additional work include:

1. Estimation of numerical uncertainty of the calculations,

2. Increase resolution of third direction computations to investigate three-dimensional effects,

3. Apply validation metrics of numerical results against the experimental data. 


\section{Part 2: Preliminary CFD Simulations of the VHTR Lower Plenum}

The objective of this study was to model a section of the VHTR prismatic reference design lower plenum using two different CFD codes and compare their results to experimental data obtained in the Idaho National Laboratory (INL) Matched-Index-of-Refraction (MIR) facility. The experimental data will comprise a validation data set to assess the applicability of CFD models to analysis of flow in the VHTR lower plenum. The progress to date for the CFD predictions of flow through a flow test model representing a section of the VHTR lower plenum is reported herein. Analysis of flow was performed for the simplified case of an unheated, constant property fluid. This analysis neglects buoyancy-driven flow effects and is considered representative of normal full-power operation [Condie, et al., 2005].

As discussed in Section 2, a computational model was constructed to replicate the flow test model. Two CFD codes, FLUENT ${ }^{\circledR}$ [Fluent, 2005] and NPHASE [Antal, et. al, 2000], were run for the identical geometry and inlet conditions. NPHASE employs a steady-state RANS computation with a standard $\mathrm{k} \sim \varepsilon$ turbulence model. URANS computations with a realizable $\mathrm{k} \sim \varepsilon$ turbulence model were run using FLUENT $^{\circledR}$. The computations in this section of the report focus on a sub-region of the lower plenum, modeled as an array of five cylindrical support posts and half-posts in a confined channel with flow entering through four inlet jets above the plenum.

This section of the report presents preliminary results from the CFD computations, including velocity, pressure, and turbulence quantities. Recommendations for the experiments are also given. Information obtained from these simulations will be used for the final CFD solutions and to guide experimentation. The reader is cautioned not to accept these results as final, rather to treat them as preliminary results. The final set of experimental data was not available in time to be incorporated into the inlet profiles, so assumed data profiles were used. Once the final data is available, the methodology is in place to use actual test data.

The following sub-sections present the work completed to date as part of this study. Sub-section 3.1 describes the experiment and flow test model. Sub-section 3.2 provides details on the construction of the model geometry and the generation of several computational meshes. Sub-section 3.3 discusses the CFD solver setup and the flow computations. Sub-section 3.4 presents the preliminary results. Subsection 3.5 outlines areas for further study. Sub-section 3.6 summarizes the results of this preliminary study.

\subsection{Experiments}

Experimental data for a representative section of the lower plenum are needed to validate computational models. Benchmark data are being obtained using the world's largest MIR flow system located at the INL. The MIR uses an optical technique, particle image velocimetry (PIV), to obtain nonintrusive flow measurements. The PIV system provides instantaneous and ensemble-averaged velocities at discrete points in the flow.

The flow test model depicted in Figure 17 was constructed using quartz columns and side walls, since quartz has the same index of refraction as the mineral oil used as the working fluid of the MIR system. The experiment is designed to simulate the flow in the central portion of the lower plenum, away from the outlet duct. The source of flow entering this region comes from jets exiting short coolant ducts at the corners of the hexagonal blocks, represented in the flow test model as a series of inlet jets located above the plenum. 


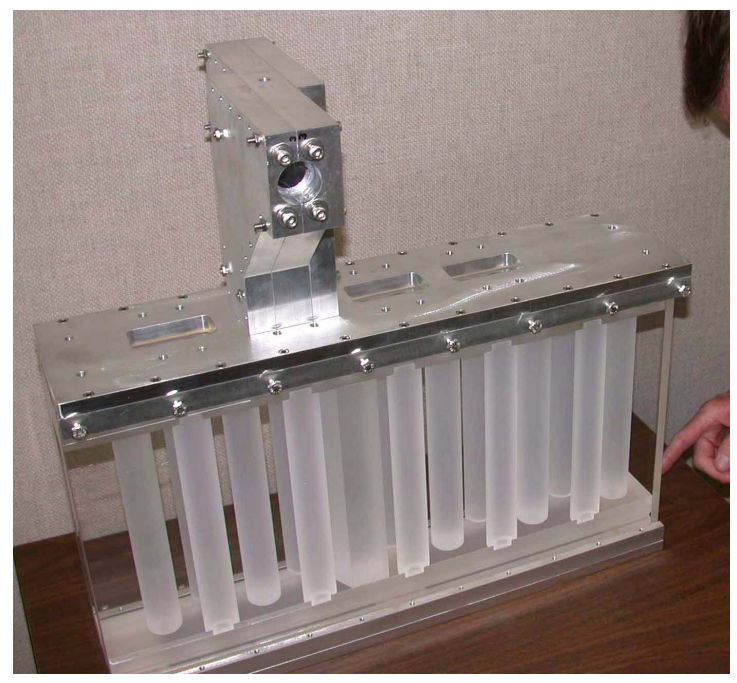

Figure 17. MIR test model.

The dimensions of the flow test model are shown in Figure 18. The model consists of eight inlet jet ports above a symmetrical arrangement of five cylindrical columns along the centerline and ten halfcolumns along the two parallel side walls. The spacing between the posts in the flow test model is $1 / \mathrm{d}=2.94$, where 1 is the streamwise distance between post centers and $\mathrm{d}$ is the post diameter. Only four of the eight inlet jets were operated during the experiments. The columns extend throughout the full height of the model. A solid wedge-shaped element at the upstream end simulates the hexagonal support block for the outer reflector. Cross flow from the main tunnel flow is blocked by the wedge. Inlet jet \#1 is blocked over one-third of its area due to the presence of the wedge. As shown in Figure 18, the jets are numbered consecutively from right to left.

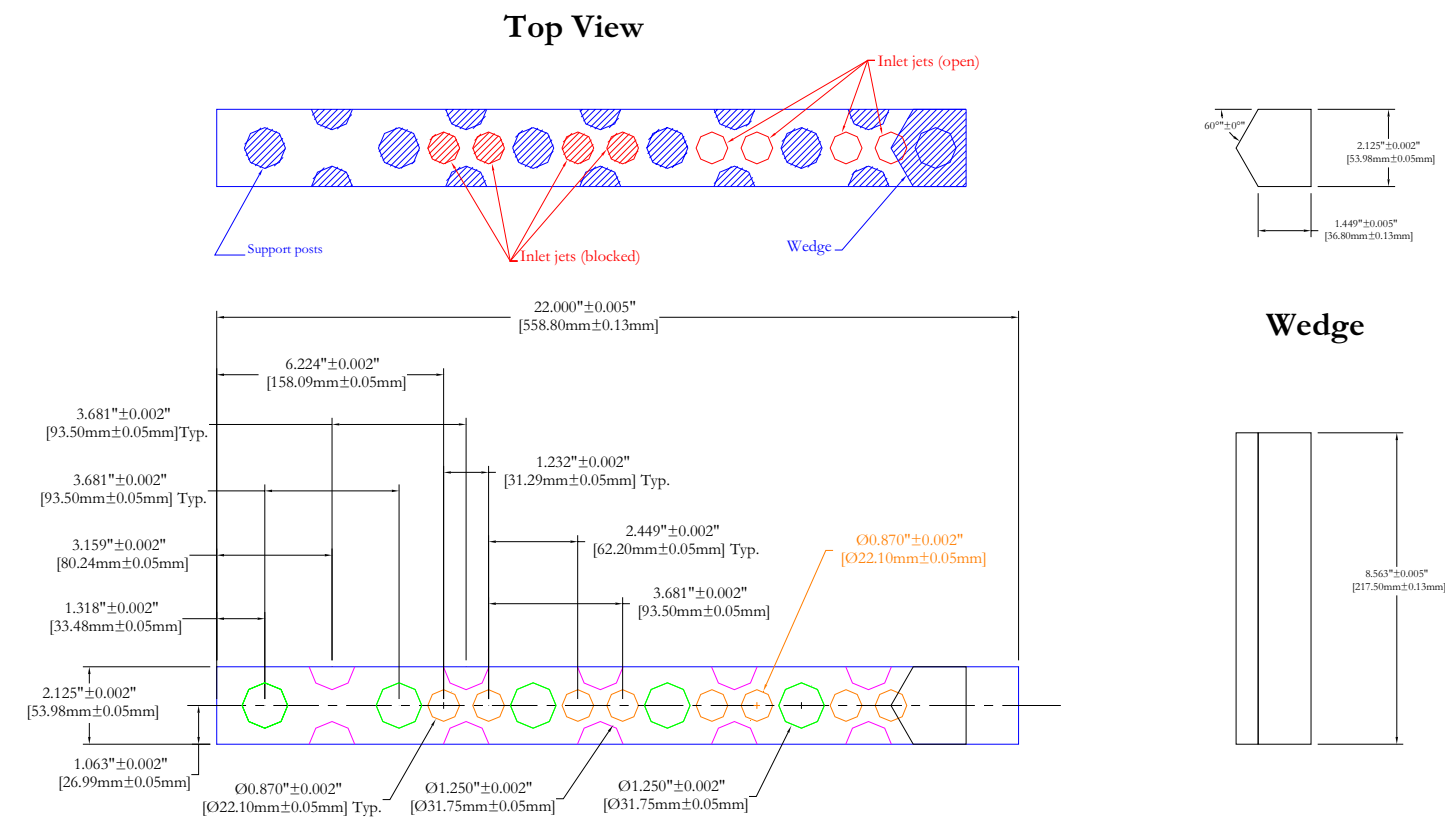

(a) 


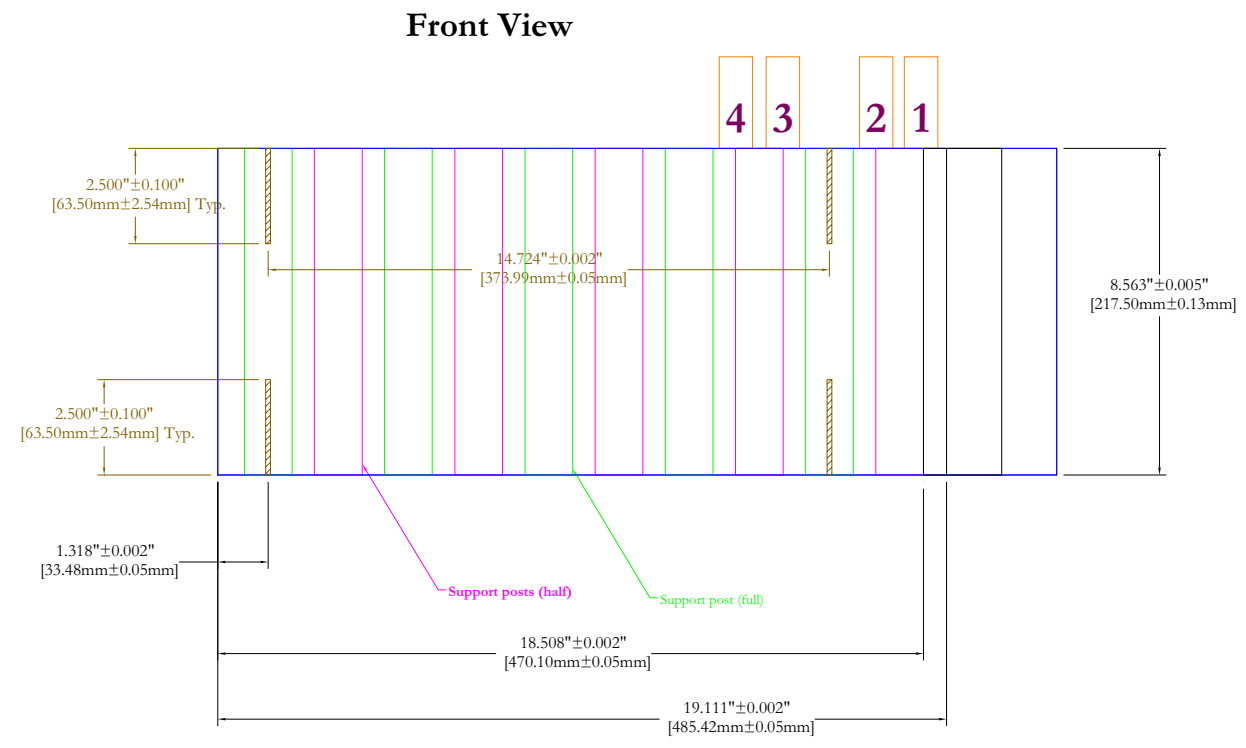

(b)

Figure 18. Flow test model dimensions (a) top view, and (b) front view.

Scaling studies have been performed to ensure that the scaled model with mineral oil flow under isothermal conditions duplicates the pertinent non-dimensional parameters [Condie, et al., 2005]. The data will be part of a validation database used to assess CFD predictions of the velocity and turbulence fields. Results from the MIR experiments will be used to generate boundary profiles for the inlet jets. These data will include $\mathrm{x}-, \mathrm{y}-$, and z-velocity and turbulent kinetic energy (TKE) over the inlet surfaces.

The temperature of the mineral oil is precisely controlled and maintained at $23.3{ }^{\circ} \mathrm{C}$ during the experiments. Unheated MIR experiments provide data for the baseline case of negligible buoyancy and constant fluid properties. These experiments are a first step to assess the fidelity of the CFD simulations. A systematic approach to validating computational predictions of turbulent mixing in the lower plenum is being implemented, wherein separate effects are examined before attempting to validate a much more complex, coupled problem using CFD. Once such flows can be computed with confidence, integrated effects will be examined.

\subsection{Grid Generation}

\subsubsection{Software}

The Pointwise software, Gridgen (version 15.09, Release 3) [Gridgen, 2006], was used to generate three-dimensional grids representative of the flow test model. NPHASE is designed to import grids generated with Gridgen, and FLUENT ${ }^{\circledR}$ is compatible with grids generated in Gridgen, as well as meshing software provided by a variety of vendors. The grids shown in this report were generated using a zero cost, trial license of Gridgen. The meshing software works best when run on a local machine, rather than on the high performance computing enclave over a network connection, to avoid unacceptably high lag times.

The FLUENT $^{\circledR}$ package consists of two separate programs, FLUENT ${ }^{\circledR}$ and Gambit [Gambit, 2006]. FLUENT $^{\circledR}$ is the flow solver (and post-processor), and Gambit is the grid generator (pre-processor).

Gambit (version 2.2.30) was used for geometry creation, mesh generation, mesh quality examination, and 
boundary zone assignment compatible with the FLUENT ${ }^{\circledR}$ flow solver. As part of this study, the capability was developed to convert a mesh created with Gambit to a format that NPHASE can read. An intermediate step of reading the Gambit mesh into FLUENT ${ }^{\circledR}$ then writing it out as in Fieldview unstructured data format was necessary. The grid was then read into mesum (the NPHASE preprocessor) via a custom $\mathrm{C}$ language program. This capability was used for the initial meshes generated with Gambit and is available to support further studies where a license to operate the Gridgen software may not be available.

Although front-end software in NPHASE permits the use of multiblock structured meshes, the author of NPHASE promotes the use of unstructured meshes with the code. The professed advantages are: "rapid grid generation, ability to generate quality meshes for complex geometries, economy of elements and more forthcoming adaptive refinement" [Antal, et al., 2000]. However, Fluent, Inc. recommends the use of structured meshes whenever possible since they afford more rapid convergence, improved accuracy and better resolution of wall boundary layers [Fluent, 2006]. The original plan was to generate a common set of grids for use in evaluating the two CFD codes. However, after initial computational results were examined it was evident that different meshes were required for two codes.

\subsubsection{Design Considerations}

The first step in the analysis process was the development of a grid that accurately approximates the flow test model and adequately resolves important flow features. The meshes incorporate design considerations discussed below.

Due to an obscuration at the jet/plenum interface, experimental data cannot be obtained in that region. Inlet jet data is available at $9 \mathrm{~mm}$ above the interface. Short circular cylinders extending $9 \mathrm{~mm}$ above the inlet jet/plenum interface were constructed in the grid to represent the inlet jets. The experimentally obtained profiles of velocity and TKE are specified at the top surface of the inlet jets, where data is available. By the time the inlet flow travels downward through the inlet jets and reaches the top of the plenum, the converged solution should satisfy the momentum equations even if the specified velocity profile did not.

An extended outlet region was added to the computational model (Figure 19) to avoid backflow at the outlet boundary. This region extends a length of $306.2 \mathrm{~mm}$ beyond the outlet plane of the test model. A grid comprised of hexagonal cells was designed to gradually coarsen from the model outlet to the computational outlet to reduce the number of nodes in the grid. 


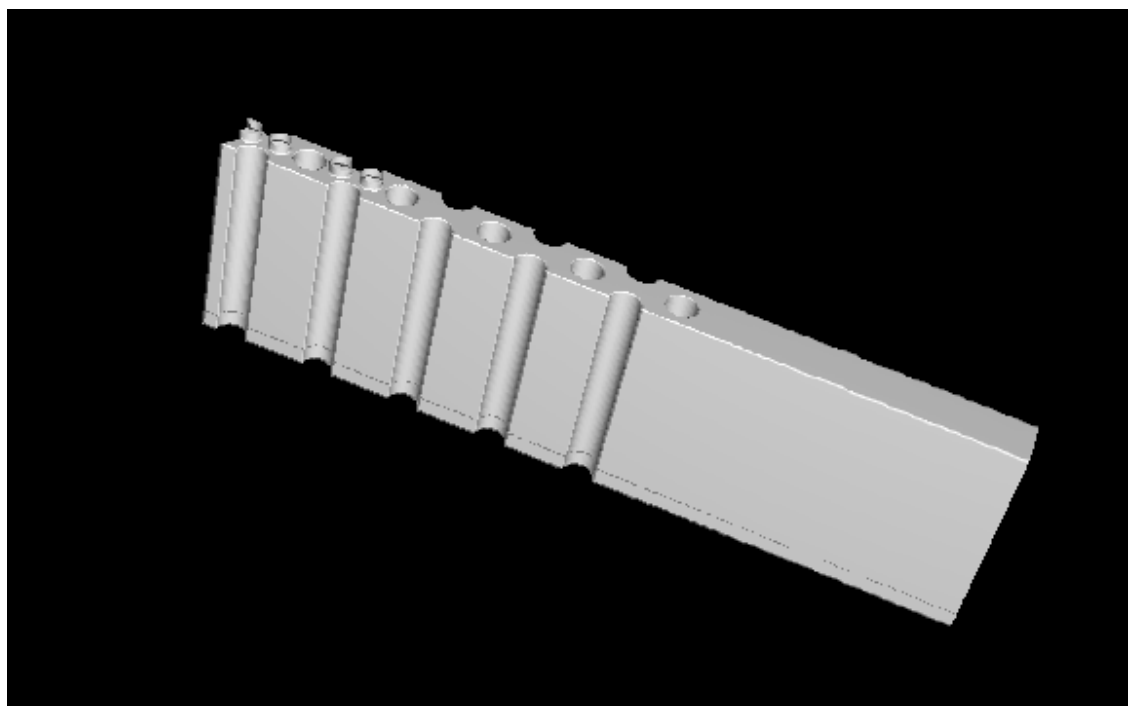

Figure 19. Computational model created for the analysis.

Fabrication drawings for the flow test model show the coordinate system origin located $36.58 \mathrm{~mm}$ upstream of the physical model (Figure 20). The computational grid was constructed to be consistent with the model dimensions and is referenced to the same origin location. This was done to facilitate comparison with the experimental data. The grids were constructed in SI units. Details of the grid construction process can be found in Gallaway [2006]. 


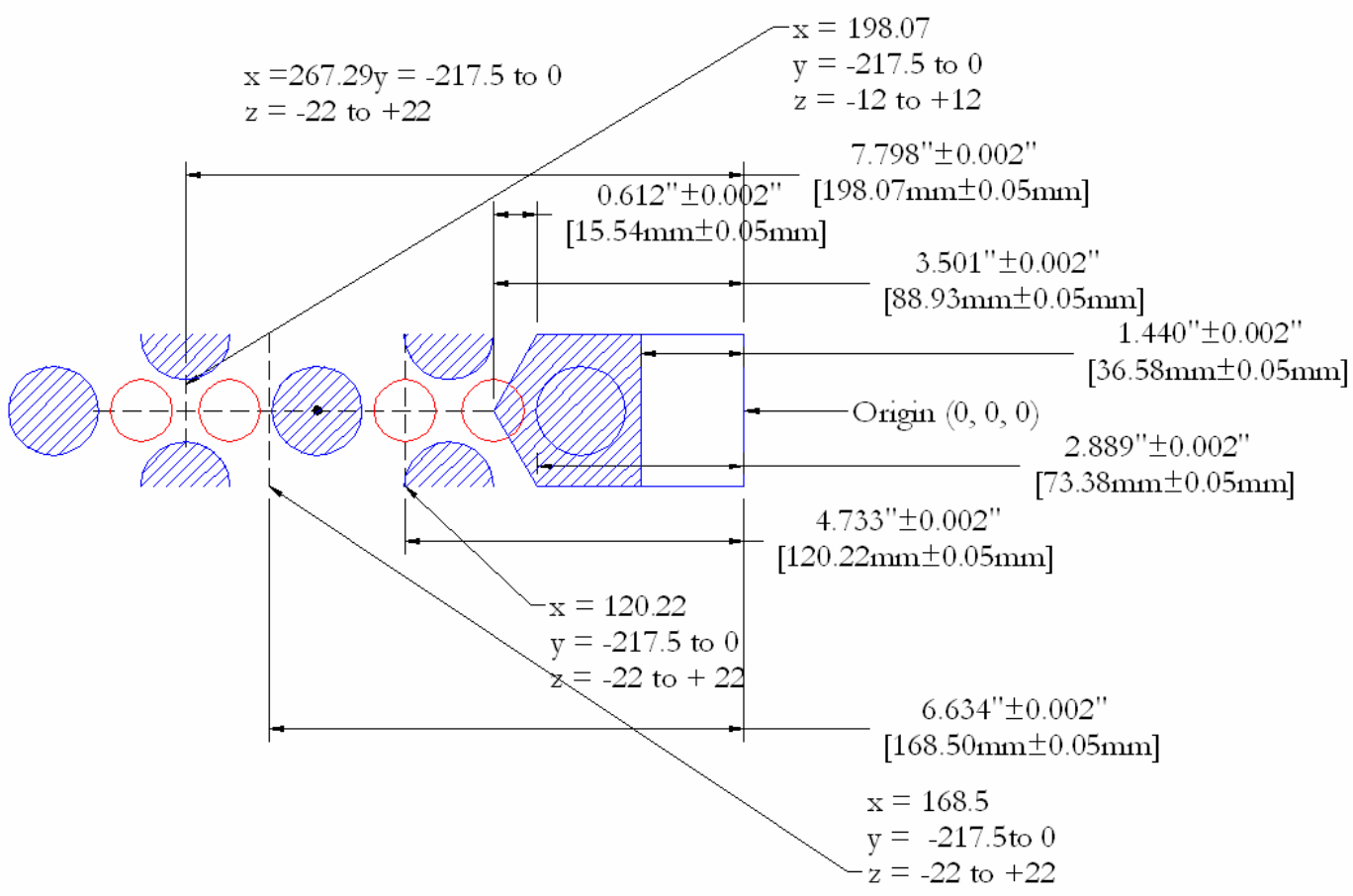

Axis Origin Location

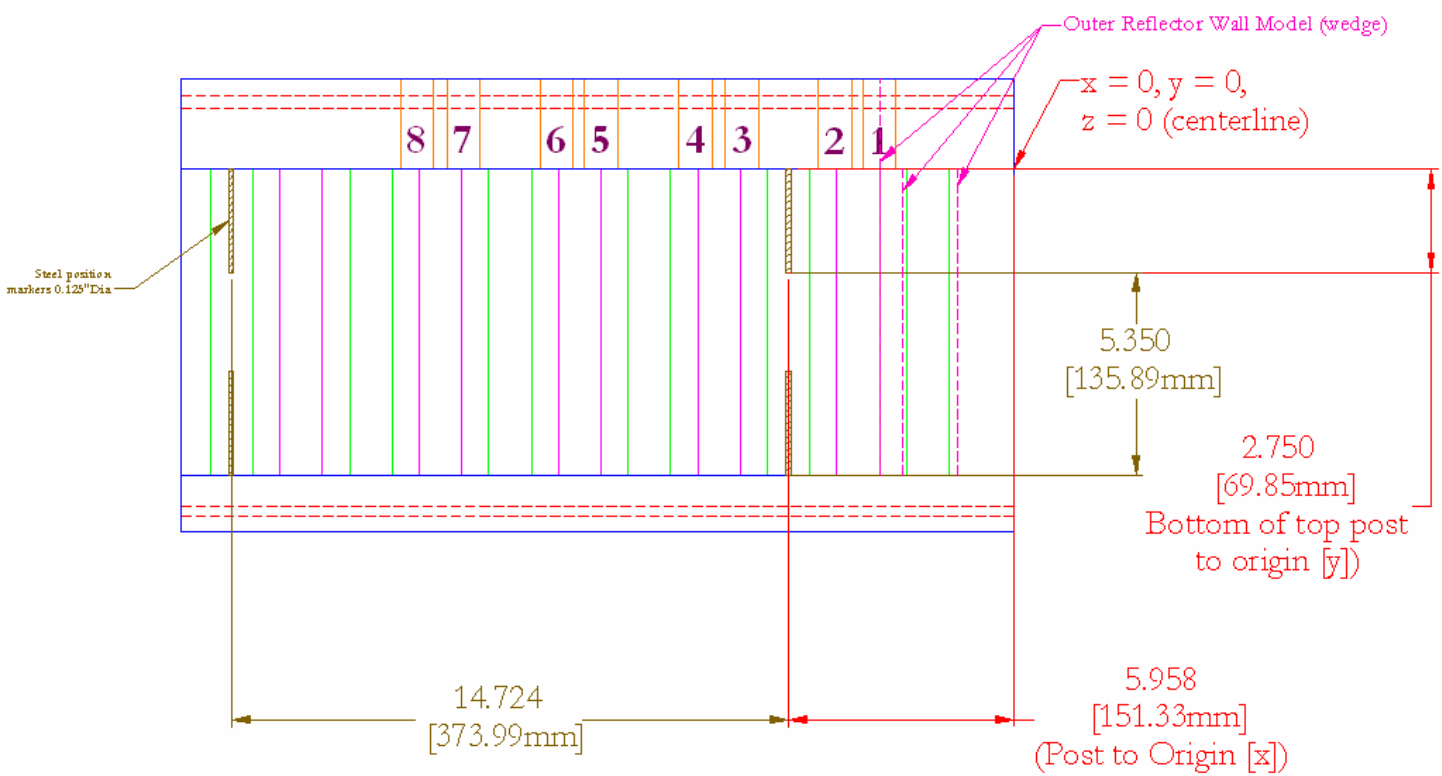

Figure 20. Lower plenum model origin location.

\subsubsection{Grid Refinement Study}

Several three-dimensional meshes were created to perform a grid convergence study. Systematic grid convergence tests are necessary to reduce numerical uncertainty by ensuring that the resolution of the computational mesh is adequate [Roache, 1998]. To determine how fine a grid resolution was needed, a 
grid study was performed using three grids of successively varying mesh refinement. "Medium," "fine," and "super-fine" meshes were generated using the Gridgen software. However, a difficulty associated with attempting to perform a grid convergence study using unstructured grids is the generation of a suitable succession of grids that retain the appropriate wall $\mathrm{y}+$ value.

FLUENT $^{\circledR}$ has the ability to use a fully unstructured grid, a fully structured grid, or a hybrid structured-unstructured grid. For the FLUENT ${ }^{\circledR}$ analysis, unstructured grids comprised of prisms were created with 225,243 cells comprising the "medium" grid (shown in Figure 21a); 839,759 cells comprising the "fine" grid (shown in Figure 21b); and 1,265,292 cells comprising the "super-fine" grid (shown in Figure 21c). Grids with over $1 \times(10)^{6}$ cells were found to be quite unwieldy to handle with current computational resources at the INL, so this was taken to be the upper limit on grid size.

A feature new to FLUENT ${ }^{\circledR}$, and only found in the 6.3 beta version, is the capability of converting an unstructured grid to a polyhedral grid. Conversion to polyhedral grids enables much faster convergence, but the user does not have control over the manner in which the cells are converted. Smaller cells are clustered near the walls, while the other regions have larger cells. Once the cells have been converted to polyhedral cells in FLUENT ${ }^{\mathbb{B}}$, they cannot be modified or adapted. If standard wall functions are used with the $\mathrm{k} \sim \varepsilon$ turbulence model, then the $\mathrm{y}^{+}$value for the first node away from the wall should be between 30 and 300 for resolution in the log-layer region. If the enhanced wall functions are used, the $\mathrm{y}^{+}$can be much smaller $(\approx 1)$. When generating the mesh, care should be taken such that the first cell adjacent to the wall is not located in the buffer layer (i.e., $\mathrm{y}^{+}$from 5 to 30 ). FLUENT ${ }^{\circledR}$ version 6.3.21 was used to convert the "medium" unstructured grid to a polyhedral grid (shown in Figure 21a) containing 328,816 cells; the "fine" unstructured grid to a polyhedral grid (shown in Figure 21b) containing 689,857 cells; and the "super-fine" unstructured grid to a polyhedral grid (shown in Figure 21c) containing $1,050,320$ cells.

A different set of grids, consisting of a combination structured mesh near the walls and an unstructured mesh in the interior, were used for the NPHASE computations. This arrangement was necessary to keep the spacing of the first grid point away from the wall the same for the various grids. The high Reynolds number $\mathrm{k} \sim \varepsilon$ model in NPHASE uses standard wall functions. For the NPHASE computations using a $\mathrm{k} \sim \varepsilon$ turbulence model, it is desired to keep the $\mathrm{y}^{+}$value between 30 and 300 . The first wall adjacent cell is located $2 \mathrm{~mm}$ from the walls, which produces a $\mathrm{y}^{+} \approx 11$ that is below the desired range for $\mathrm{y}^{+}$. The "medium" grid (shown in Figure 22a) contains 322,722 cells; the "fine" grid (shown in Figure 22b) contains 792,534 cells; and the "super-fine" grid (shown in Figure 22c) contains 1,346,934 cells. FLUENT ${ }^{\circledR}$ converged to unphysical results with this set of grids. 
Unstructured Grids
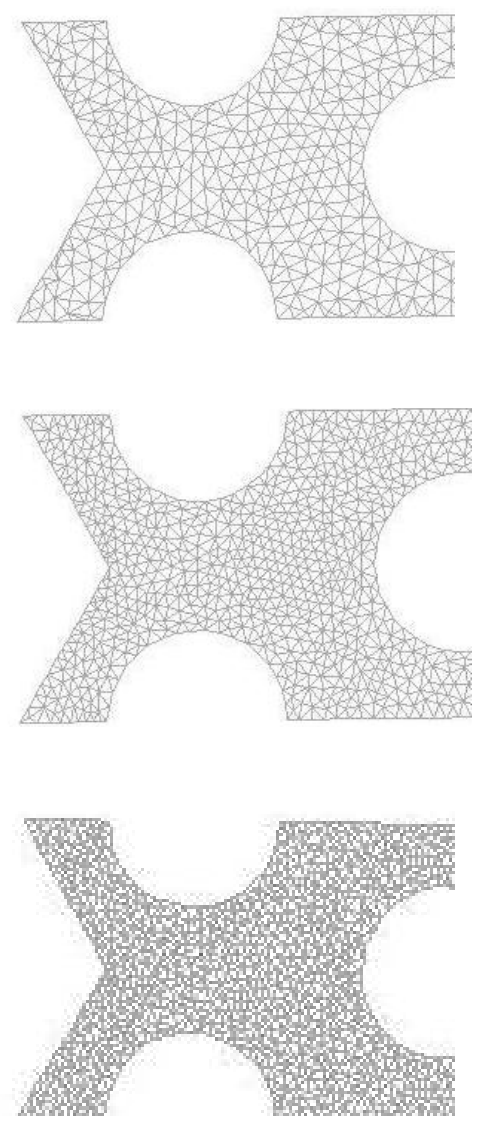

Polyhedral Grids

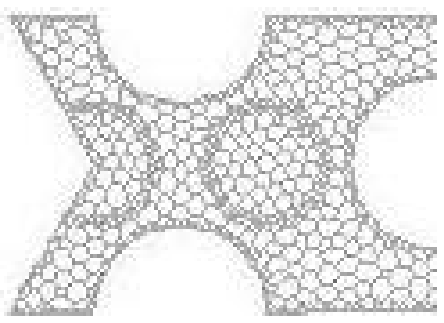

(a) "medium"

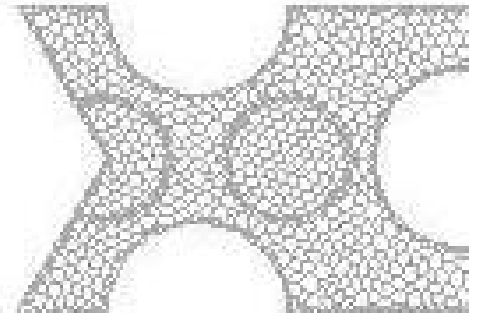

(b) "fine"

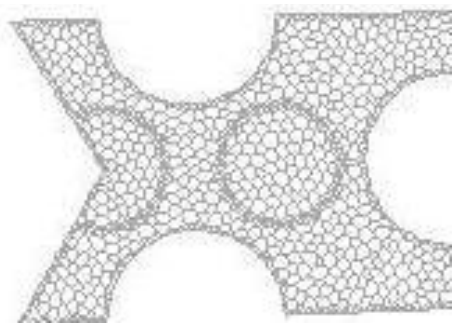

(c) "super-fine"

Figure 21. Detail sections of meshes created for grid independence study using FLUENT ${ }^{\circledR}$.

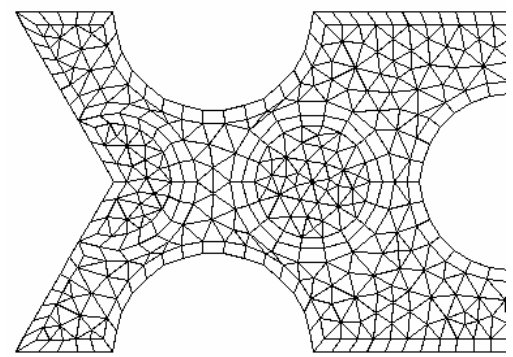

(a) "medium"

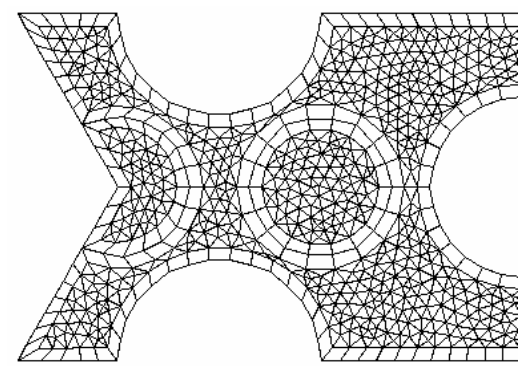

(b) "fine"

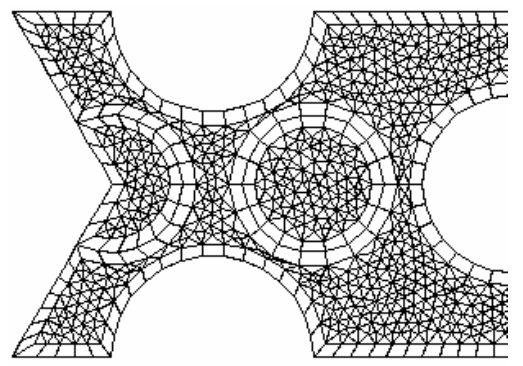

(c) "super-fine"

Figure 22. Detail section of hybrid grids created for grid independence study using NPHASE.

For three-dimensional grids, the average global cell size, h, is defined by [Celik, 2006]:

$$
\mathrm{h}=\left[\frac{1}{\mathrm{~N}} \sum_{\mathrm{i}=1}^{\mathrm{N}}\left(\Delta \mathrm{V}_{\mathrm{i}}\right)\right]^{\frac{1}{3}}
$$


Table 1 lists the average global cell size for the nine grids generated for this study. The volume of the fluid domain is $0.0075 \mathrm{~m}^{3}$. The refinement factor (i.e., $\mathrm{h}_{\text {original }} / \mathrm{h}_{\text {refined }}$ ) ranges from 1.2 to 1.6 .

Table 1. Average global cell size for the computational meshes generated for this study.

\begin{tabular}{lccc}
\hline \multirow{2}{*}{$\begin{array}{c}\text { Level of } \\
\text { refinement }\end{array}$} & \multicolumn{3}{c}{ Average global cell size, $\mathbf{h}(\mathbf{m})$} \\
\cline { 2 - 3 } & FLUENT & grids & NPHASE grids \\
\cline { 2 - 3 } & unstructured & polyhedral & hybrid \\
\hline "medium" & 0.0032 & 0.0028 & 0.0028 \\
"fine" & 0.0021 & 0.0022 & 0.0021 \\
"super-fine" & 0.0018 & 0.0019 & 0.0018 \\
\hline
\end{tabular}

The grids were carefully constructed to avoid the situation where all of the cell vertices lie on walls. This problem tends to occur in corners and creates difficulties in the flow solver because velocity gradients cannot be calculated for these cells. The grid shown in Figure 23 is an example of a grid configuration to avoid since all of the nodes on the cell at the lower left corner lie along the walls.

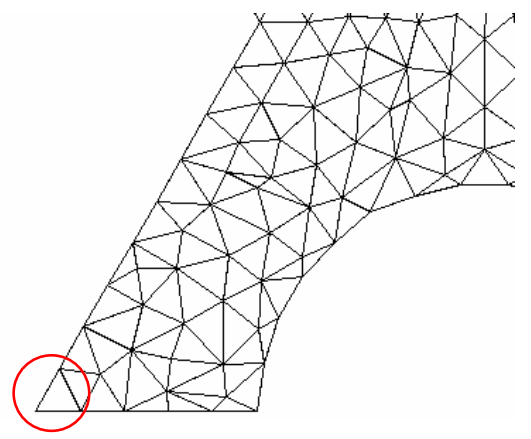

Figure 23. Example of a grid configuration to avoid.

\subsection{CFD Simulations}

\subsubsection{Description of Flow Solvers}

This section provides an overview of the NPHASE and FLUENT ${ }^{\circledR}$ CFD codes and the options selected for the flow simulation.

3.3.1.1 NPHASE Code. The NPHASE code was used in this study to perform steady-state turbulent flow simulations using the computational meshes representative of the MIR experimental model. A steady-state method was employed as a first step to gain experience running the NPHASE code. Steady-state solutions are typically less time-intensive to produce and easier to post-process and analyze than unsteady solutions. It is expected that unsteady simulations of this flow will be necessary to reproduce the time-periodic nature of the vortices in the wakes that form downstream of the cylindrical support posts.

NPHASE was originally developed as a means to accurately predict two-phase flow phenomena. However, in this study, NPHASE's ability to make accurate predictions of single phase, incompressible, turbulent flow is under consideration. NPHASE is a multiphase, multicomponent, finite-volume CFD code with the ability to analyze complex geometries, including structured or unstructured meshes. 
Individual scalar transport equations for mass, momentum, and turbulence are solved in either a coupled or segregated manner using frozen coefficient linearizations. NPHASE simulations for this study employed the segregated solver wherein the momentum equations are iteratively solved uncoupled from the continuity equation. A pressure-correction was applied to update the pressure field to support calculation of mass fluxes to ensure conservation of mass. The Semi-IMplicit Pressure-Linked Equations-Consistent (SIMPLEC) scheme was used for pressure-velocity coupling. NPHASE utilizes an algebraic multigrid solver to eliminate long wavelength error components. Second-order accurate discretizations were employed for the convection and diffusion terms [Antal, et al., 2000]. An opensource post-processing package, Parallel Visual Application (Paraview) version 2.4 by Kitware, Inc., was used to visualize NPHASE results.

3.3.1.2 FLUENT ${ }^{\circledR}$ Code. The experimental conditions were also modeled using the commercial CFD code FLUENT ${ }^{\circledR}$. The segregated solver used for this study uses a point Gauss-Seidel technique and multigrid V-cycle acceleration. This code uses a control volume technique to convert the governing equations to algebraic equations that are solved numerically. This control volume technique consists of integrating the governing equations for each control volume, yielding discrete equations that conserve each quantity on a control volume basis. FLUENT ${ }^{\circledR}$ stores discrete values of the conserved quantity at the cell centers and uses an upwind technique for determining face values of the conserved quantity for the convective terms. A second-order scheme was used for higher-order accuracy. A PREssure STaggering Option (PRESTO) scheme was used as the interpolation scheme for calculating cell-face pressures. A Pressure-Implicit with Splitting of Operators (PISO) pressure-velocity coupling scheme, which uses a combination of continuity and momentum equations to derive an equation for pressure, was also used. It is part of the SIMPLE family of algorithms. One of the limitations of the SIMPLE and SIMPLEC algorithms is that new velocities and corresponding fluxes do not satisfy the momentum balance after the pressure-correction equation is solved. As a result, the calculation must be repeated until the balance is satisfied. To improve the efficiency of this calculation, the PISO algorithm performs both neighbor and skewness correction to decrease the number of iterations required for convergence of transient problems. The use of the PISO pressure-velocity coupling scheme should lead to faster time step convergence rates when compared to SIMPLE or SIMPLEC. The Monotone Upstream-Centered Schemes for Conservation Laws (MUSCL) scheme was used to interpolate the field variables (stored at cell centers) to the faces of the control volumes. This scheme produces a locally third-order convective discretization for unstructured meshes. A time-step size of 0.002 seconds was used for the computations.

3.3.1.3 Turbulence Model. RANS simulations treat the flow variables as having a time-averaged (mean) part and a turbulent part. The latter is modeled with a turbulence model. This CFD application employs a $\mathrm{k} \sim \varepsilon$ turbulence model known for its robustness, economy and reasonable accuracy over a wide range of turbulent flows common in industry. The entire range of turbulence scales is modeled, and only mean flow features are resolved. For the NPHASE computations, the $\mathrm{k} \sim \varepsilon$ turbulence model with standard wall functions was used. The standard $\mathrm{k} \sim \varepsilon$ model is the only turbulence model available in NPHASE. For the FLUENT ${ }^{\circledR}$ study, the realizable $\mathrm{k} \sim \varepsilon$ (where $\mathrm{k}$ is TKE and $\varepsilon$ is the TKE dissipation rate) turbulence modeling option with enhanced wall treatment was used. The realizable $\mathrm{k} \sim \varepsilon$ model shows substantial improvements over the standard $\mathrm{k} \sim \varepsilon$ model for problems where the flow features include strong streamline curvature and vortices [Fluent, 2006]. The $\mathrm{k} \sim \varepsilon$ turbulence model solves for total TKE assuming turbulent viscosity is isotropic. However, the generation of TKE due to mean flow gradients may be different depending on which mean flow velocity gradients are being considered. It is a challenge for the CFD model to contain a fine enough mesh to fully simulate the physical conditions in the experimental model, including the boundary layers on the walls. The goal here was to determine whether a $\mathrm{k} \sim \varepsilon$ turbulence model and "super-fine" grid resolution is sufficient. This was a first level approach for the CFD simulations since substantially more computing resources are needed for higher order turbulence models. Future work should assess the performance of other turbulence closure models, including the 
k $\sim \omega$ model, Reynolds Stress Model (RSM), and Large Eddy Simulation (LES), within the framework of unsteady solutions.

3.3.1.4 Iterative Convergence Criteria. Residuals of mass, momentum, TKE, and $\varepsilon$ were monitored to determine iterative convergence. In FLUENT ${ }^{\circledR}$, these residuals are normalized values. The URANS solution was allowed to iterate until the residuals reached $1 \times(10)^{-6}$ for mass and momentum and $1 \mathrm{x}(10)^{-5}$ for TKE and $\varepsilon$. These convergence tolerances were based on the research performed in Section 2 of this report. The net difference in mass flux through the inlets and outlet is $4 \times(10)^{-8}$ for calculations performed on the "fine" unstructured mesh and $2.9 \times(10)^{-7}$ for the "fine" polyhedral mesh. Steady-state RANS simulations were also run using the "fine" grid, but these solutions did not converge to acceptable levels. After 30171 iterations, the residuals were $3.68 \times(10)^{-4}$ for continuity, $3.42 \times(10)^{-5}$ for $\mathrm{x}$-velocity, $6.60 \times(10)^{-5}$ for $y$-velocity, $1.93 \times(10)^{-5}$ for z-velocity, $3.14 \times(10)^{-5}$ for TKE, and $1.07 \times(10)^{-4}$ for $\varepsilon$. The steady-state solution scheme employed a SIMPLEC algorithm for pressure-velocity coupling, a standard discretization scheme for pressure, and a first-order discretization scheme for momentum, TKE, and $\varepsilon$. The under-relaxation factor for momentum was decreased from 0.7 to 0.5 , but this was not sufficient to achieve convergence. Perhaps the reason that the steady-state solutions did not converge is that the flow is inherently unsteady.

In NPHASE, the residuals are not normalized, they are absolute units based on the root mean square (RMS) change in the variables. The NPHASE solution was determined to be converged when the residuals for velocity reached $1 \mathrm{x}(10)^{-6} \mathrm{~m} / \mathrm{s}$.

3.3.1.5 Boundary Conditions, Initial Conditions, and Material Properties. The working fluid in the MIR facility is mineral oil. Material properties for mineral oil were specified in the flow solver set up (e.g., a density of $831 \mathrm{~kg} / \mathrm{m}^{3}$ and a dynamic viscosity of $0.0118 \mathrm{~kg} / \mathrm{m} \cdot \mathrm{s}$ ).

Preliminary results from the MIR experiments are being used to generate boundary profiles for the inlet jets. The inlet boundary conditions for mean velocity and TKE for the CFD model were set to match the experimental data. These data include average $\mathrm{x}-, \mathrm{y}-$, and z-velocity and TKE over the inlet surface needed for the $\mathrm{k} \sim \varepsilon$ turbulence model. The CFD model needs initial values for $\varepsilon$, which is not measured in the MIR facility. This issue can be overcome through some trial and error applications of the CFD model. Some work toward this goal has been done but more remains. The initial value for $\varepsilon$ is specified to be $0.01 \mathrm{~m}^{2} / \mathrm{s}^{3}$. FLUENT ${ }^{\mathbb{B}}$ and NPHASE simulations execute successfully with either constant inlet conditions or with the experimental profiles obtained in the MIR facility for jets 3 and 4. Figures 24a and $24 \mathrm{~b}$ depict contours of the preliminary inlet jet velocity and TKE data for an inlet jet Reynolds number of 4300. Since experimental data for inlet jets 1 and 2 was not available, data from jet 3 was used for inlet jet 1 and 2 profiles. Once the final data is provided, the inlet data profiles can be updated and the simulations re-run. The inlet velocity profiles have the shape of fully developed turbulent velocity profiles. The TKE data should be zero in the vicinity of the walls. The non-zero TKE is attributed to the inaccuracy of PIV near the walls.

The inlet jet data was input into FLUENT $^{\circledR}$ using the boundary profiles feature. A user routine written in the $\mathrm{C}$ programming language was developed to input the inlet jet profiles into NPHASE. Before running the user routine, the experimental data must first be interpolated onto the grid points that comprise the inlet jets since the locations at which the measurements were made do not coincide with the mesh point locations. 


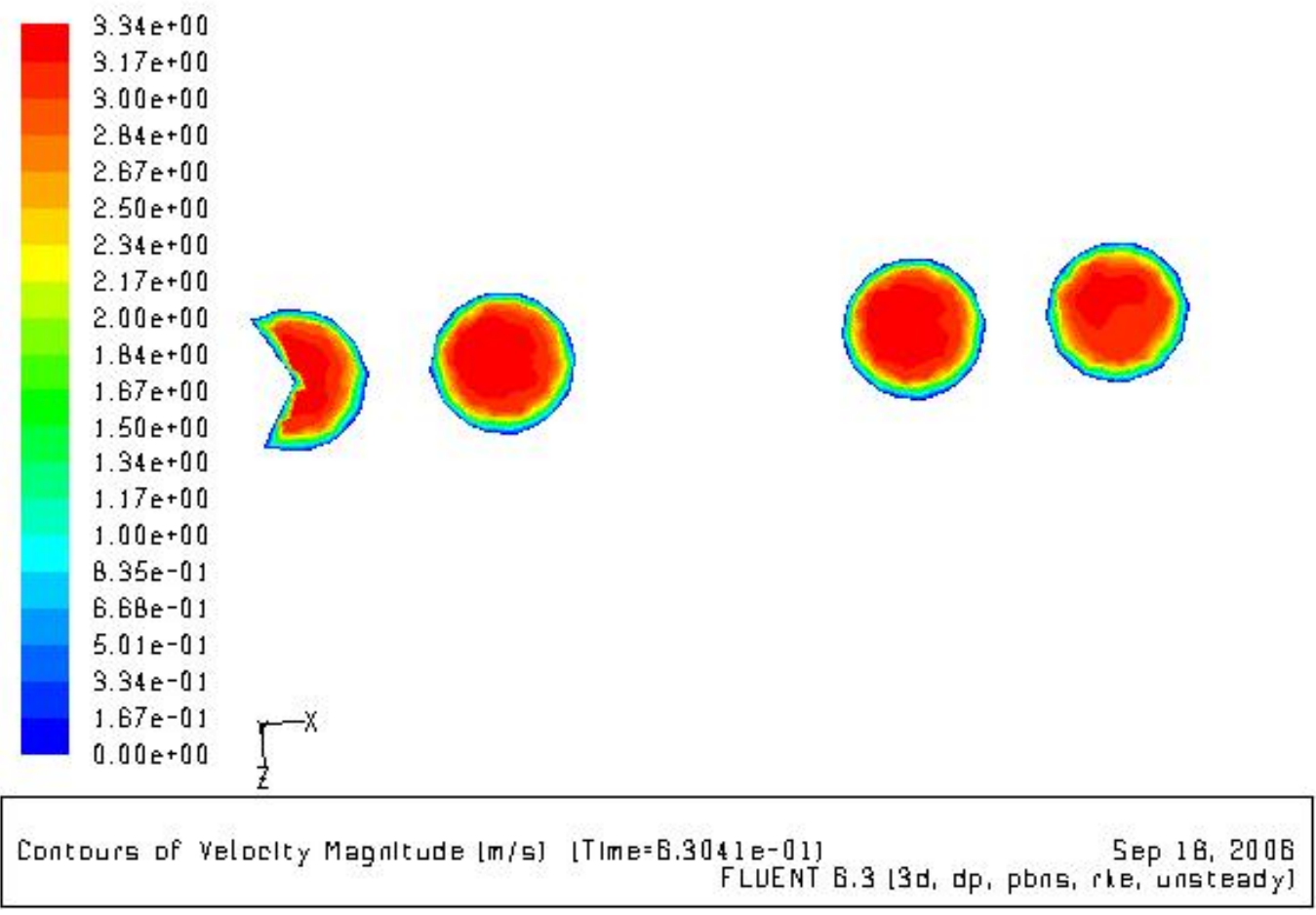

Figure 24(a). Top view of inlet jets \#1 through 4 - contours of velocity magnitude.

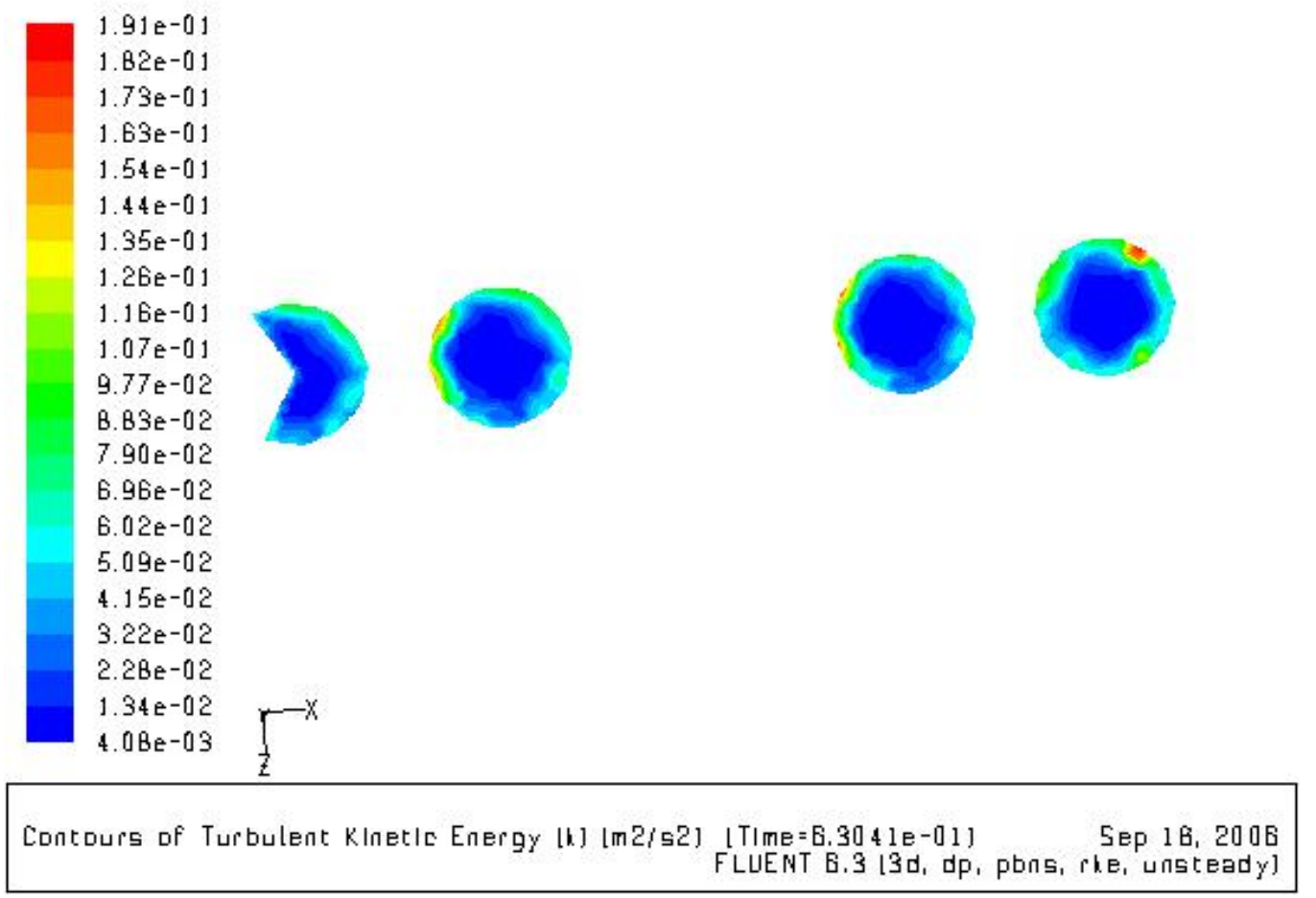

Figure 24(b). Top view of inlet jets \#1 through 4 - contours of TKE.

Wall boundary conditions were specified for the surfaces of the circular cylinders and half cylinders; the channel sides, top, and bottom; the hexagonal wedge; and the sides of the inlet jets. A no- 
slip condition was enforced at the walls. The flow outlet was specified as a constant pressure outlet. The backflow TKE was set to $0.01 \mathrm{~m}^{2} / \mathrm{s}^{2}$, and $\varepsilon$ was set to $0.01 \mathrm{~m}^{2} / \mathrm{s}^{3}$.

The initial condition was specified wherein the $\mathrm{x}-, \mathrm{y}-$, and $\mathrm{z}$ - components of velocity and the static gage pressure were set to zero (i.e., $\mathrm{V}_{\mathrm{x}}=\mathrm{V}_{\mathrm{y}}=\mathrm{V}_{\mathrm{z}}=\mathrm{P}=0$ ). However, the effect of different initial conditions should be evaluated, especially when using a $\mathrm{k} \sim \varepsilon$ turbulence model [Rumsey, et al., 2006] to ensure that the equations do not converge to a non-unique solution.

\subsection{Results from CFD Simulations}

Steady-state NPHASE computations and unsteady FLUENT ${ }^{\circledR}$ computations are currently being completed. Preliminary results are illustrated to show the progress made to date.

\subsubsection{NPHASE Results Displayed with Paraview}

Due to time and hardware constraints, converged NPHASE solutions were only obtained for the "medium" and "fine" grids with two jets running. Contour plots for both grids with a constant inlet velocity of $-3.2 \mathrm{~m} / \mathrm{s}$ and TKE of $0.02 \mathrm{~m}^{2} / \mathrm{s}^{2}$ are displayed in Figures 25 and 26 . By comparing results from both grids, it is possible to observe the effects of grid refinement. Subtle differences can be observed in the velocity contour for each grid on the left side of the geometry, just before the jets. Pressure ranges for each grid differ slightly by a few hundred Pascals. This may occur due to stronger pressure gradients around central columns in the finer grid. Flow separates at the center columns, and then reconnects to pass through the side columns. This flow pattern will cause subtle pressure gradients observed in the contour plots, particularly after the third central column, to form. The maximum pressure is located on the plenum floor beneath inlet jet \#4.

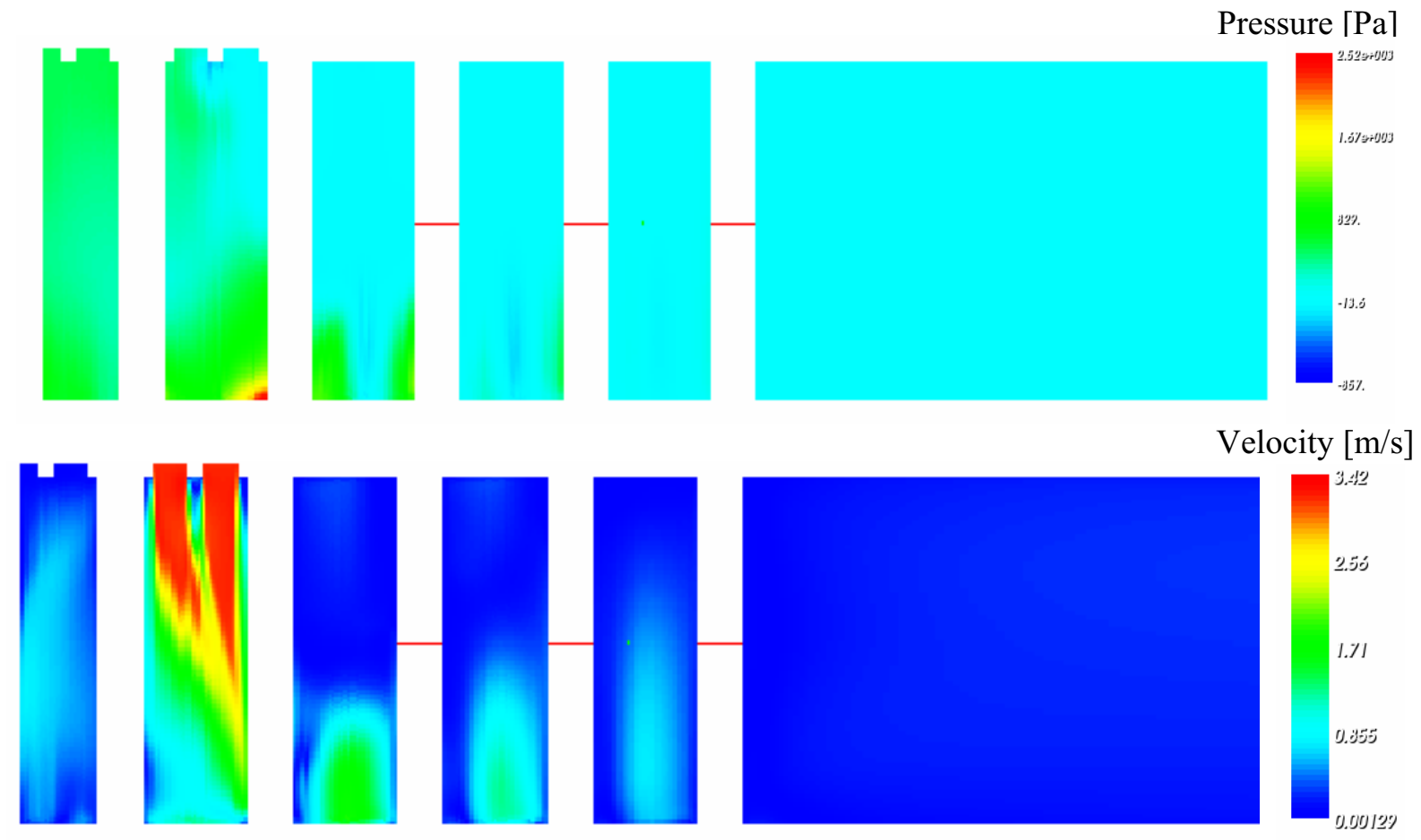

Figure 25. Pressure and velocity distributions assuming constant inlet velocity and turbulence quantities for the "medium" grid. 

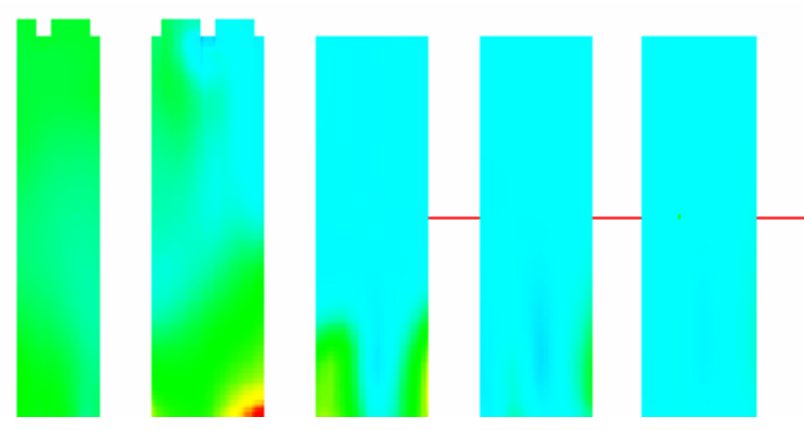

Pressure $[\mathrm{Pa}]$
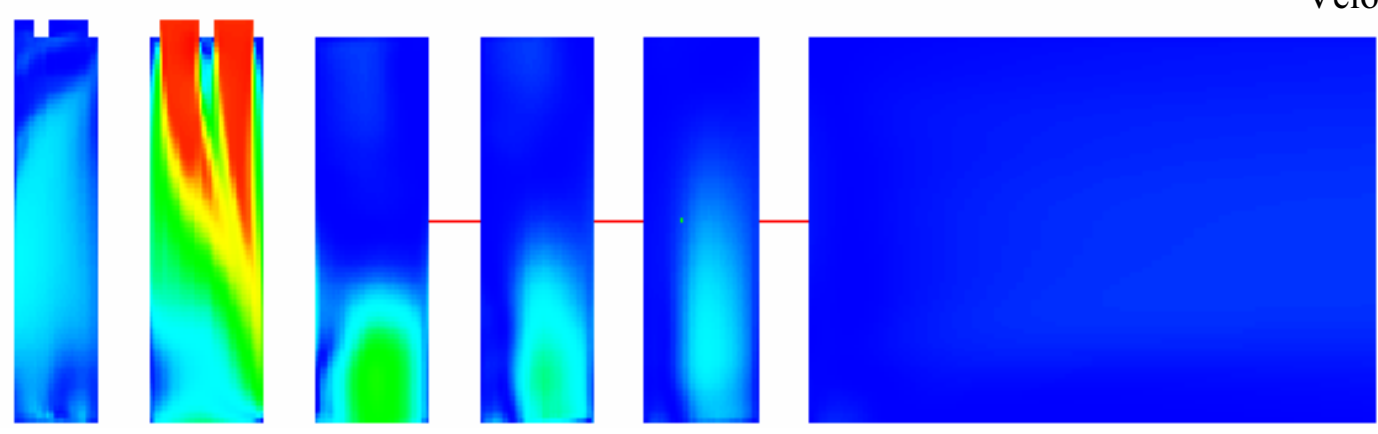

Velocity $[\mathrm{m} / \mathrm{s}]$

Figure 26. Pressure and velocity distributions assuming constant inlet velocity and turbulence quantities for the "fine" grid.

Figures 27 and 28 display contour plot results from CFD simulations with time-averaged, experimental inlet conditions for the "medium" and "fine" grid. Distinctions between the two solutions for the different grids are similar to those observed for the constant inlet velocity cases. The variations in velocity are smaller than in Figures 25 and 26, which can be partially attributed to a lower mass flow rate. The average velocity across the inlets is approximately $2.5 \mathrm{~m} / \mathrm{s}$ for the cases with the profiles, versus 3.2 $\mathrm{m} / \mathrm{s}$ for cases with constant inlet velocity. However, the reason why the region of higher velocity does not extend farther into the plenum needs to be examined further. It is possible that the flow did not reach steady-state conditions or that the experimental profiles were not input correctly. Pressure ranges in the profiled plots also differ significantly from those in the constant inlet conditions plots. Pressure ranges below vary from just over $1 \mathrm{kPa}$ above atmospheric pressure $\left(\mathrm{P}_{\mathrm{atm}}\right)$ to less than $1 \mathrm{kPa}$ below $\mathrm{P}_{\mathrm{atm}}$.

For comparison, Figure 28b shows converged solutions obtained using the FLUENT $^{\circledR}$ code with the "super-fine" unstructured grid and the inlet profiles. There are marked differences between the NPHASE and the FLUENT ${ }^{\circledR}$ solutions. The effect of the inlet jet flow is seen as a region of higher velocity beneath the inlet jets penetrating into the top half of the plenum. Regions of relatively higher static pressure are located at the floor of the plenum beneath the inlet jets. Comparison with the experimental data will guide further simulations. 
Pressure $[\mathrm{Pa}]$
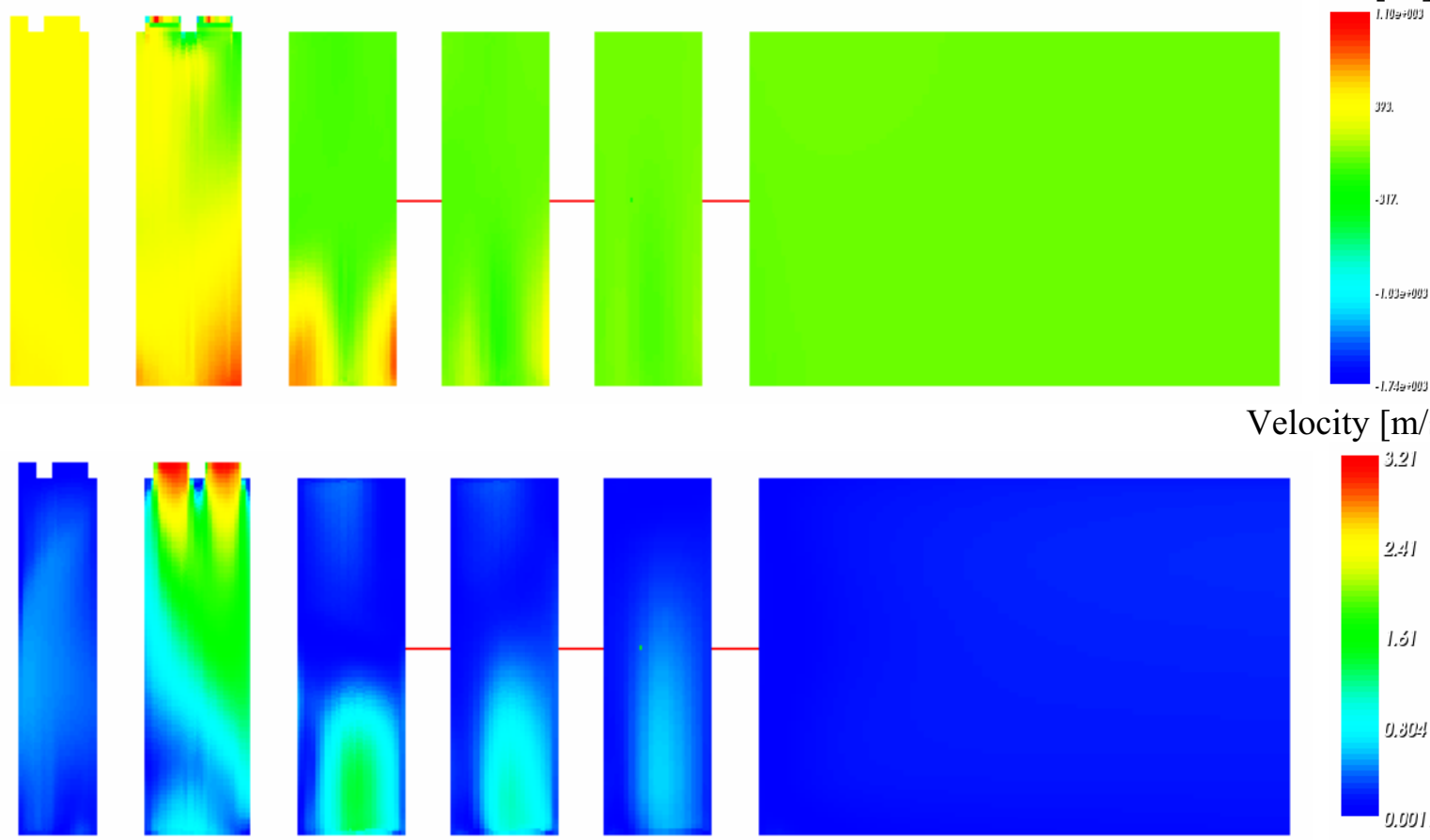

Velocity $[\mathrm{m} / \mathrm{s}]$

Figure 27. Pressure and velocity distributions using experimental profiles for inlet velocity and

turbulence quantities for the "medium" grid.

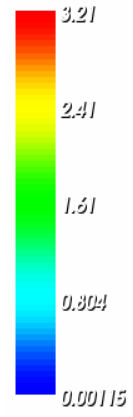

Pressure $\lceil\mathrm{Pa}\rceil$
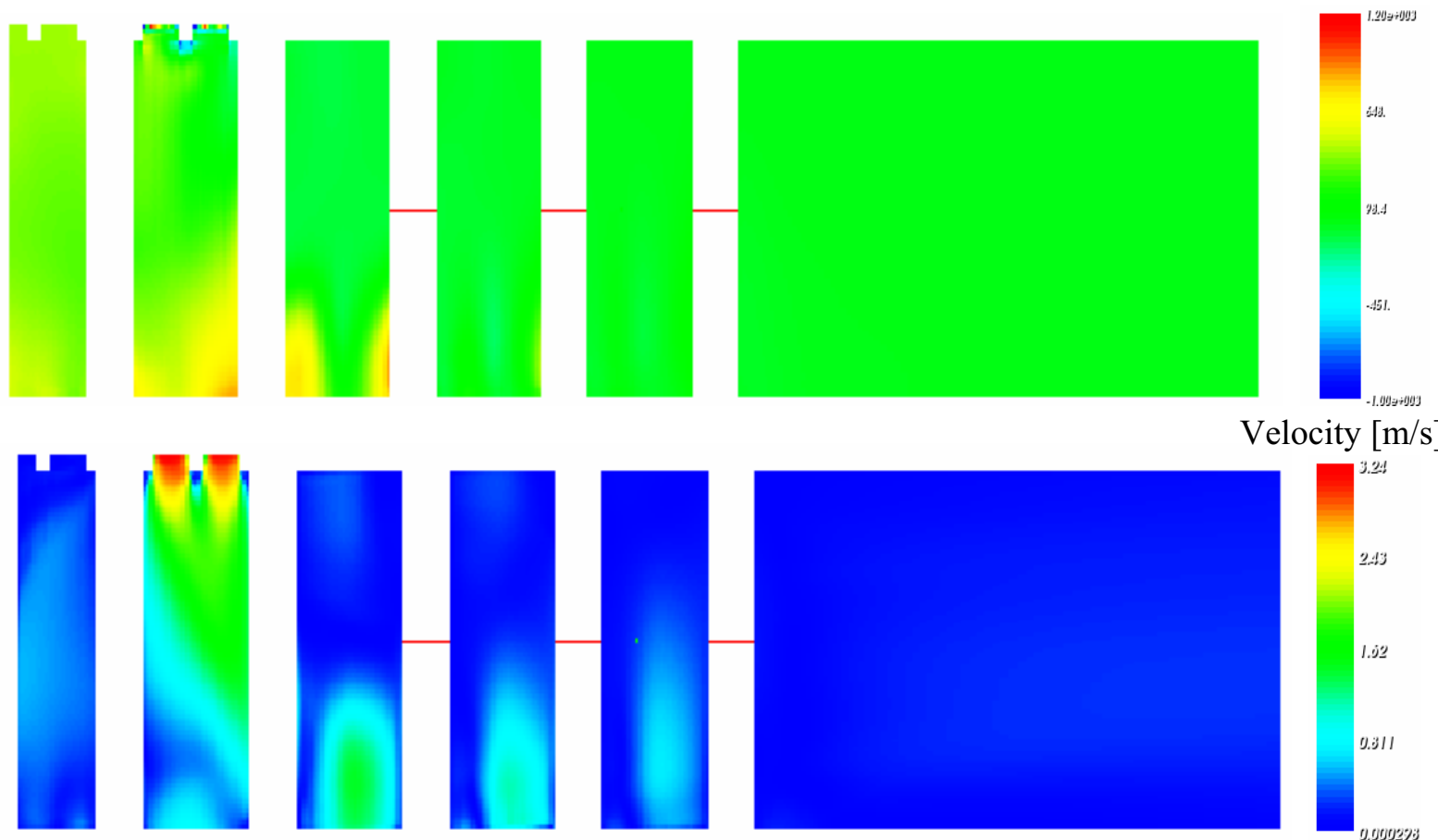

Velocity $[\mathrm{m} / \mathrm{s}]$

Figure 28(a). Pressure and velocity distributions using experimental profiles for inlet velocity and turbulence quantities for the "fine" grid using NPHASE. 


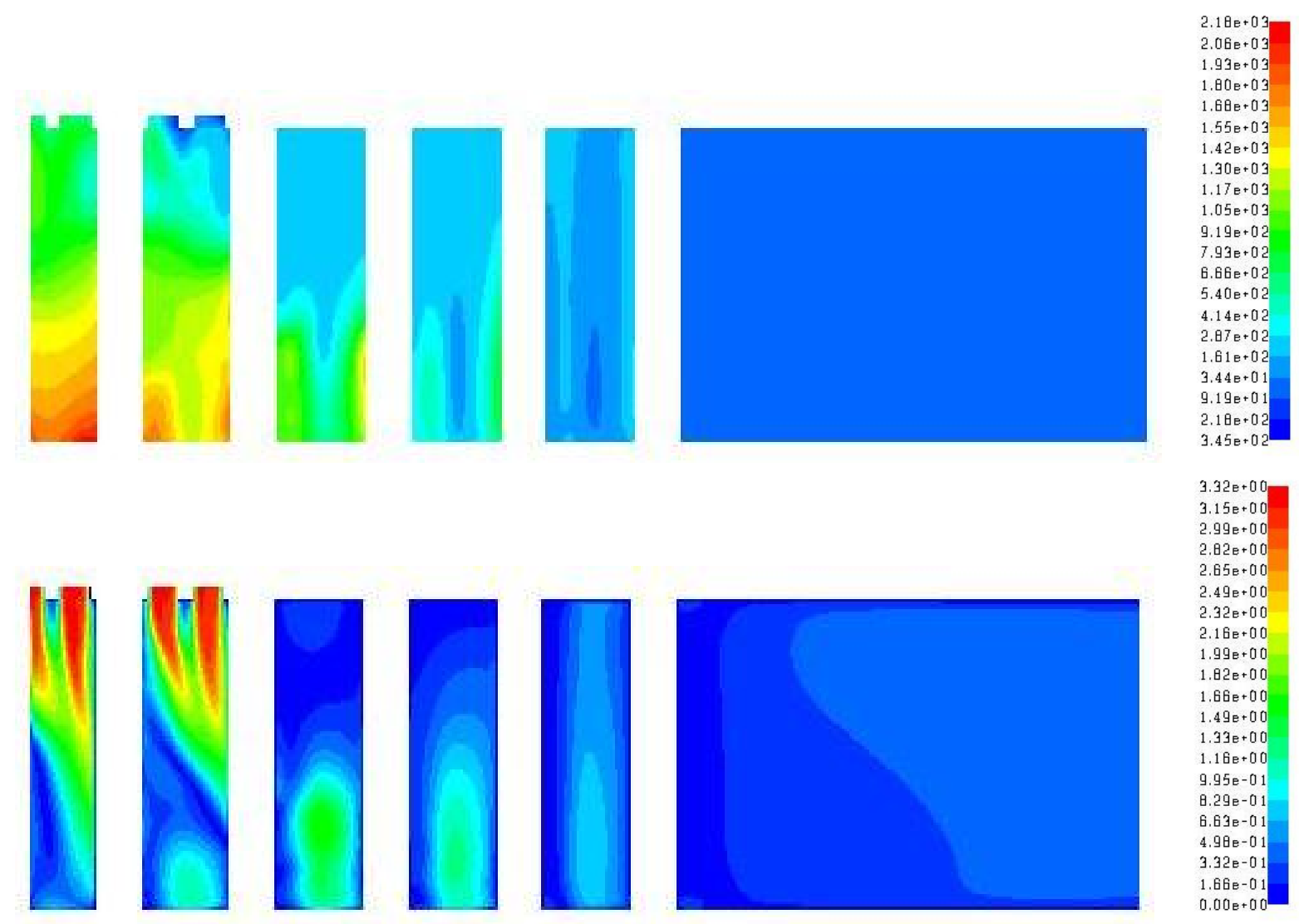

Figure 28(b). Pressure and velocity distributions using experimental profiles for inlet velocity and turbulence quantities for the "super-fine" grid using FLUENT ${ }^{\circledR}$.

\subsection{2 $\quad$ FLUENT $^{\circledR}$ Results}

The results of the URANS computations produced using the FLUENT ${ }^{\circledR}$ code are presented in the following sub-sections.

3.4.2.1 Contour Plots of FLUENT ${ }^{\circledR}$ Results. Figures 29-35 show contours of mean x-, y-, and z-velocity; velocity magnitude; static pressure; TKE; and turbulence intensity (TI) on the x-y plane along the model centerline $(\mathrm{z}=0)$. Table 2 lists the minimum and maximum values of these quantities produced over all cells in the "fine" unstructured grid at one instant during the simulation. The color maps in Figures 29-40 are based upon the local values of the surfaces shown in the contour plots.

Table 2. Minimum and maximum values in the VHTR lower plenum flow simulation.

\begin{tabular}{lcc}
\hline & Minimum & Maximum \\
\hline $\mathrm{V}_{\mathrm{x}}(\mathrm{m} / \mathrm{s})$ & -0.852 & 1.793 \\
$\mathrm{~V}_{\mathrm{y}}(\mathrm{m} / \mathrm{s})$ & -3.341 & 1.184 \\
$\mathrm{~V}_{\mathrm{z}}(\mathrm{m} / \mathrm{s})$ & -1.001 & 0.995 \\
$\mathrm{~V}(\mathrm{~m} / \mathrm{s})$ & 0. & 3.34 \\
$\mathrm{P}(\mathrm{Pa})$ & -707.4 & 2199.2
\end{tabular}




$\begin{array}{lcr}\text { TKE }\left(\mathrm{m}^{2} / \mathrm{s}^{2}\right) & 6.13 \mathrm{x}(10)^{-8} & 0.911 \\ \text { Wall y }^{+} & 0.155 & 14.3 \\ \text { TI }(\%) & 0.08 & 21.6\end{array}$

The contour plots shown in Figures 29-31 for x-, y-, and z-velocity confirm that the flow is threedimensional. The regions of highest velocity magnitude (shown in Figure 32) are at and directly below the inlet jets. Since the inlet flow exits into a plenum with much larger flow area, by mass conservation it is expected that the flow velocity in the plenum is lower.

Figure 29 depicts computed contours of $\mathrm{x}$-velocity along the centerline of the test model $(\mathrm{z}=0)$. The presence of the hexagonal wedge at the upstream end of the model blocks cross flow from the main flow stream in the tunnel. Strong recirculation regions can be seen on the floor of the lower plenum beneath inlet jets 1 and 2 and in front of the hexagonal wedge; downstream of support cylinder \#1 roughly midway along the height of the plenum; and along the top of the lower plenum just downstream of support cylinder \#2. Weaker recirculation regions are present in the wake region just downstream of each cylindrical support post; on the model floor just downstream of each post; and at the top of the model just downstream of post \#5.

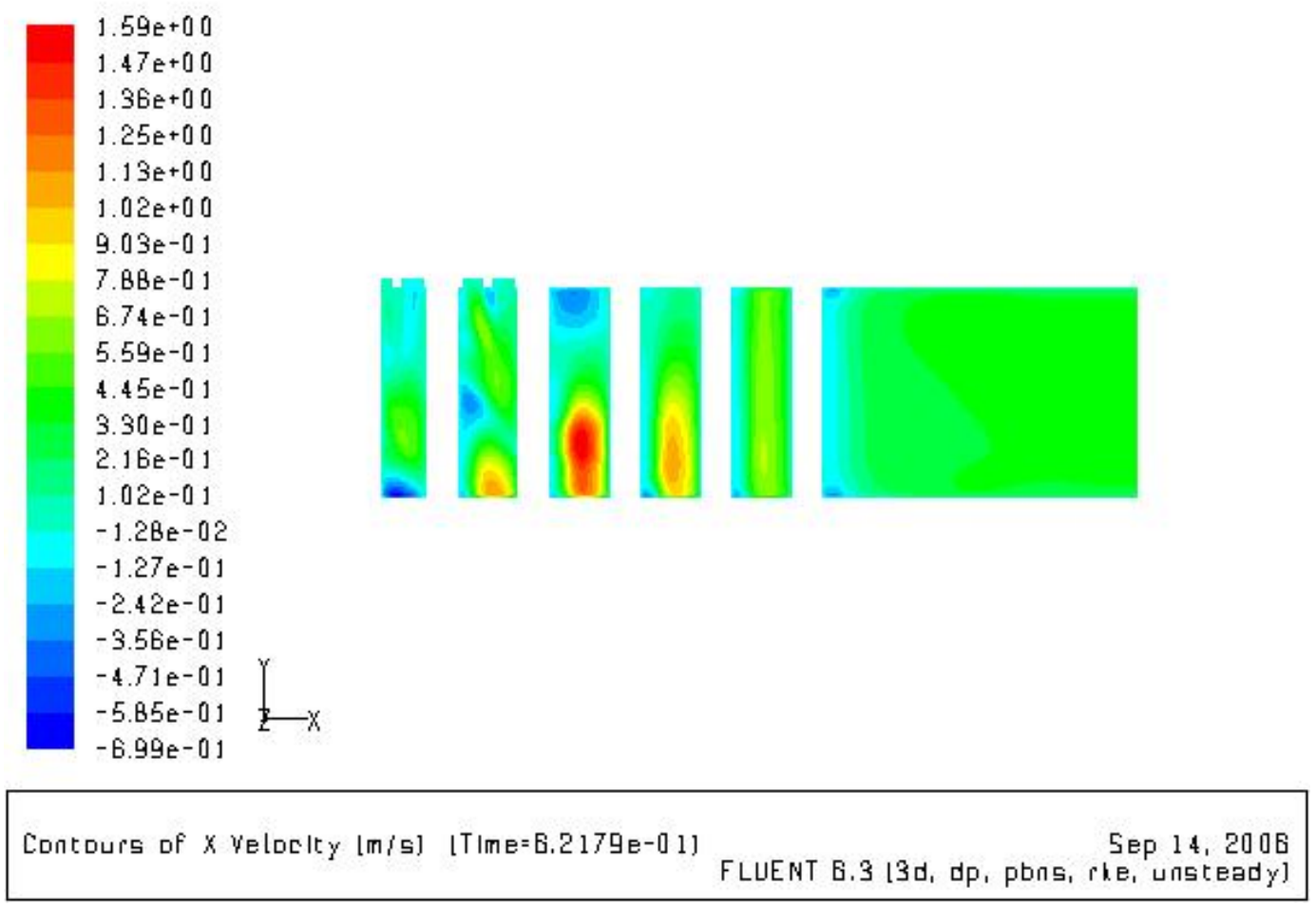

Figure 29. Computed contours of $x$-velocity on the $x-y$ plane along the centerline $(z=0)$.

The flow exits the inlets and enters the test section with principally a negative y-component of velocity (shown in Figure 30). The flow gradually turns, and the x-component of velocity increases as the fluid flows around post \#2. Since the flow area is reduced in this location, the flow velocity increases. The flow converges behind post \#2 and passes between the third set of half-posts. The maximum xvelocity occurs in the lower half of the plenum between posts \#2 and \#3. 
Regions of large negative y-velocity (Figure 30) occur in the upstream section of the plenum, directly underneath the four inlet jets. Beyond post \#2, the y-velocity is small. The magnitude of zvelocity (Figure 31) is small throughout the plenum and is fairly constant downstream of post \#3 to the extended outlet.

Figures 32 and 33 show the Bernoulli effect as the flow accelerates through the area constriction caused by the half-posts. In these regions, the static pressure is lower as a result of the increase in velocity.

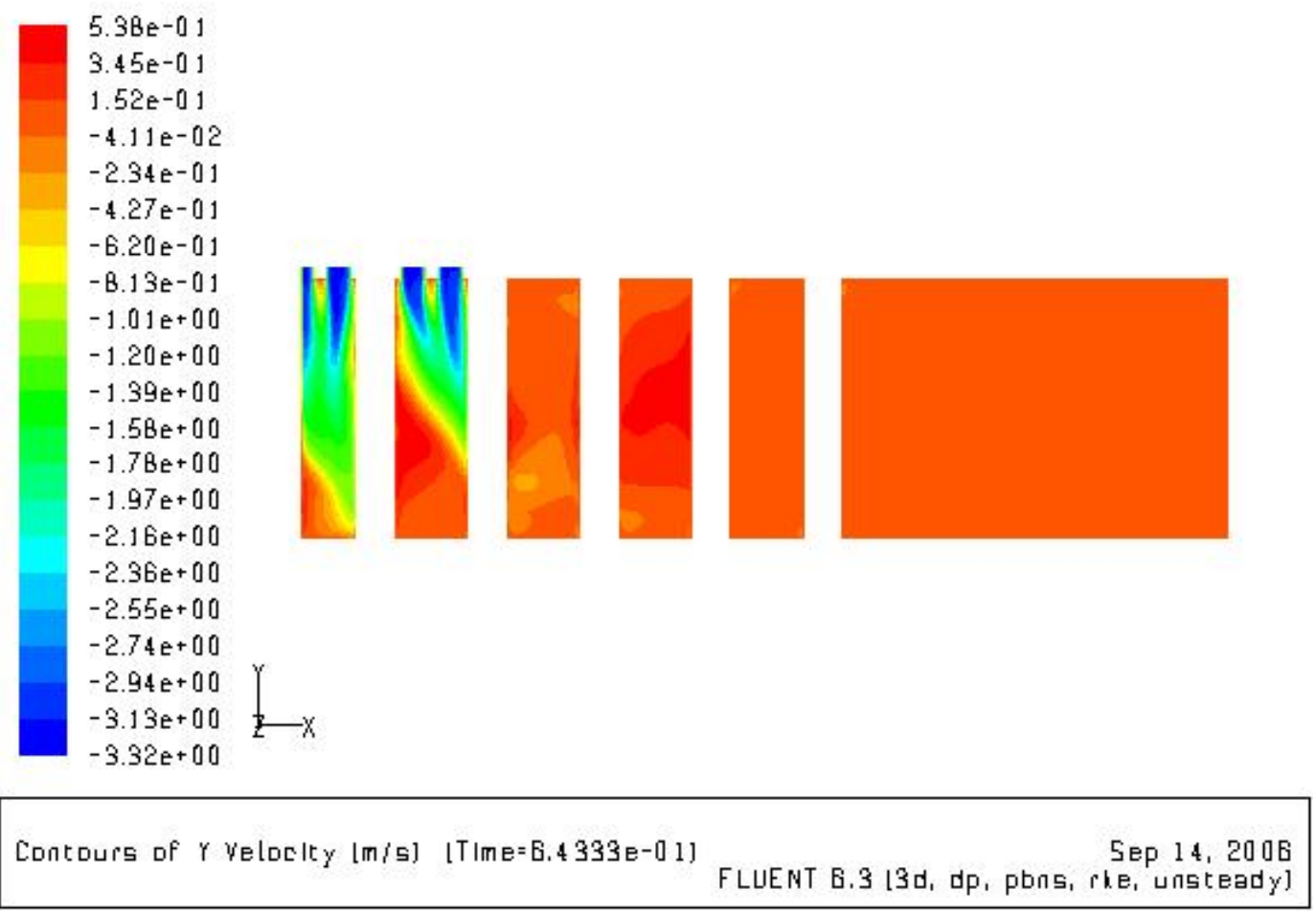

Figure 30. Computed contours of $y$-velocity on the $x-y$ plane along the centerline $(z=0)$. 


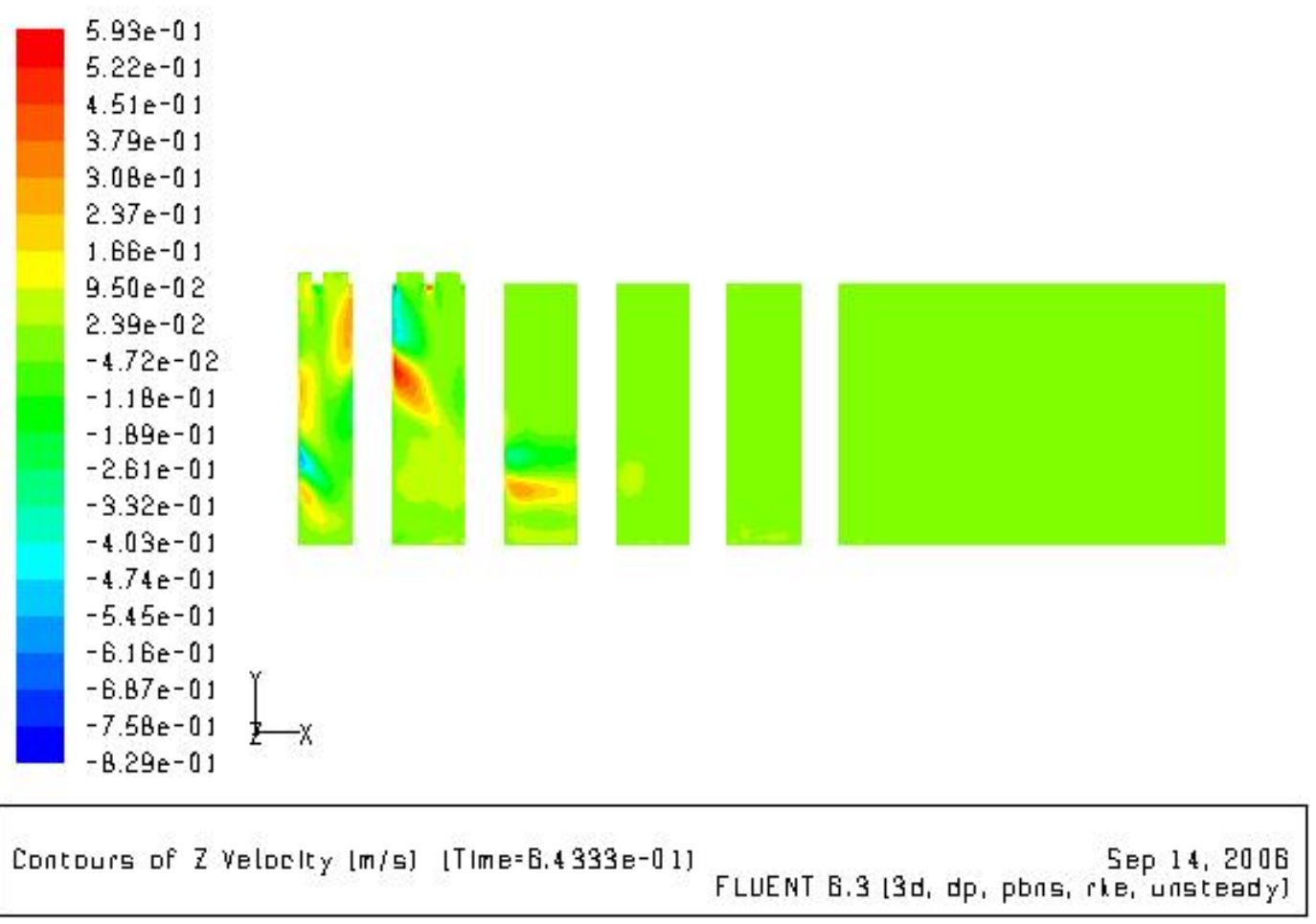

Figure 31. Computed contours of z-velocity on the $x-y$ plane along the centerline $(z=0)$.

Figure 32 shows that the region of highest pressure is located at the floor of the test model, beneath the inlet jets. The maximum static pressure occurs on the model floor beneath jet $\# 2$.

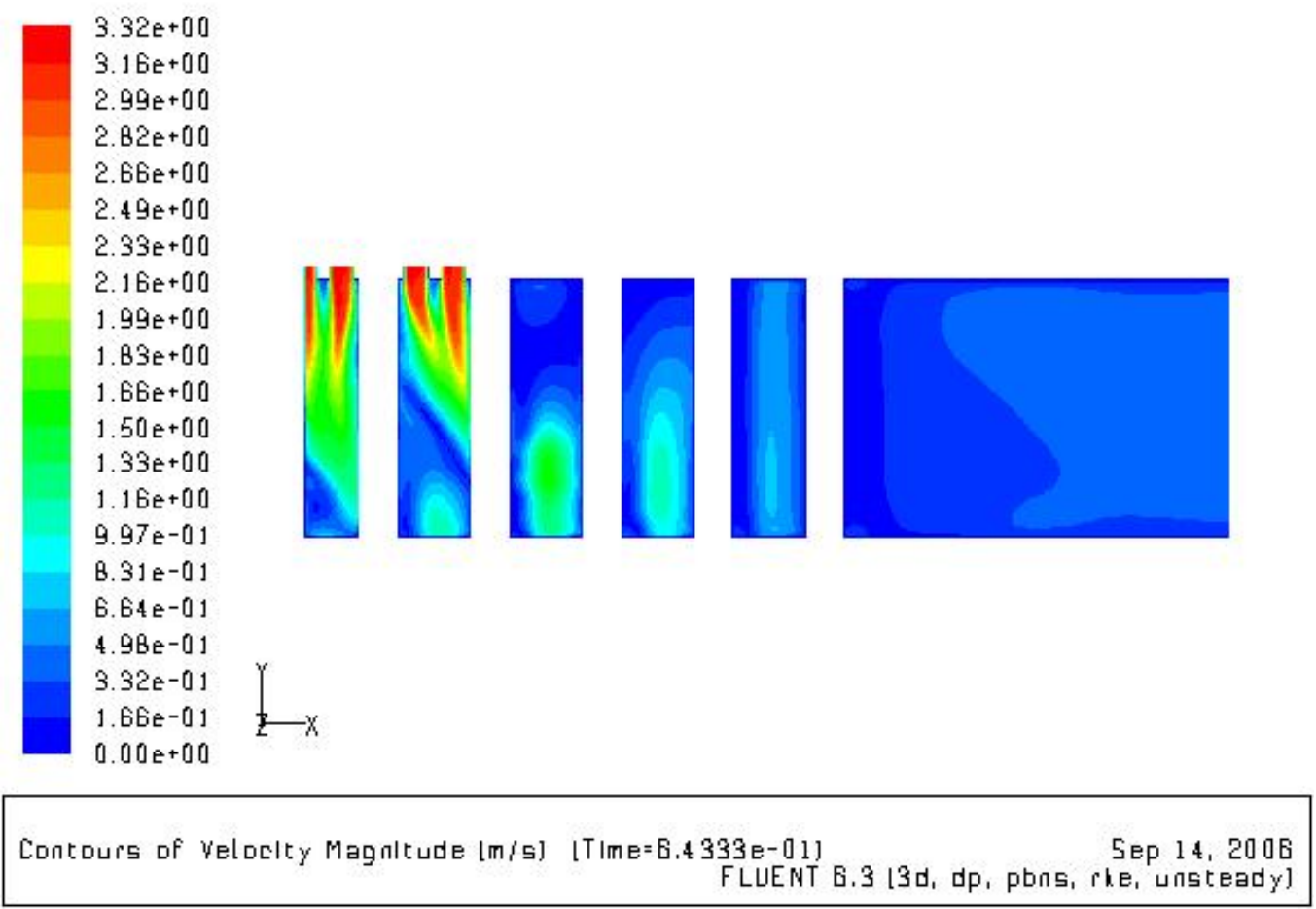

Figure 32. Computed contours of velocity magnitude on the $x-y$ plane along the centerline $(\mathrm{z}=0)$. 


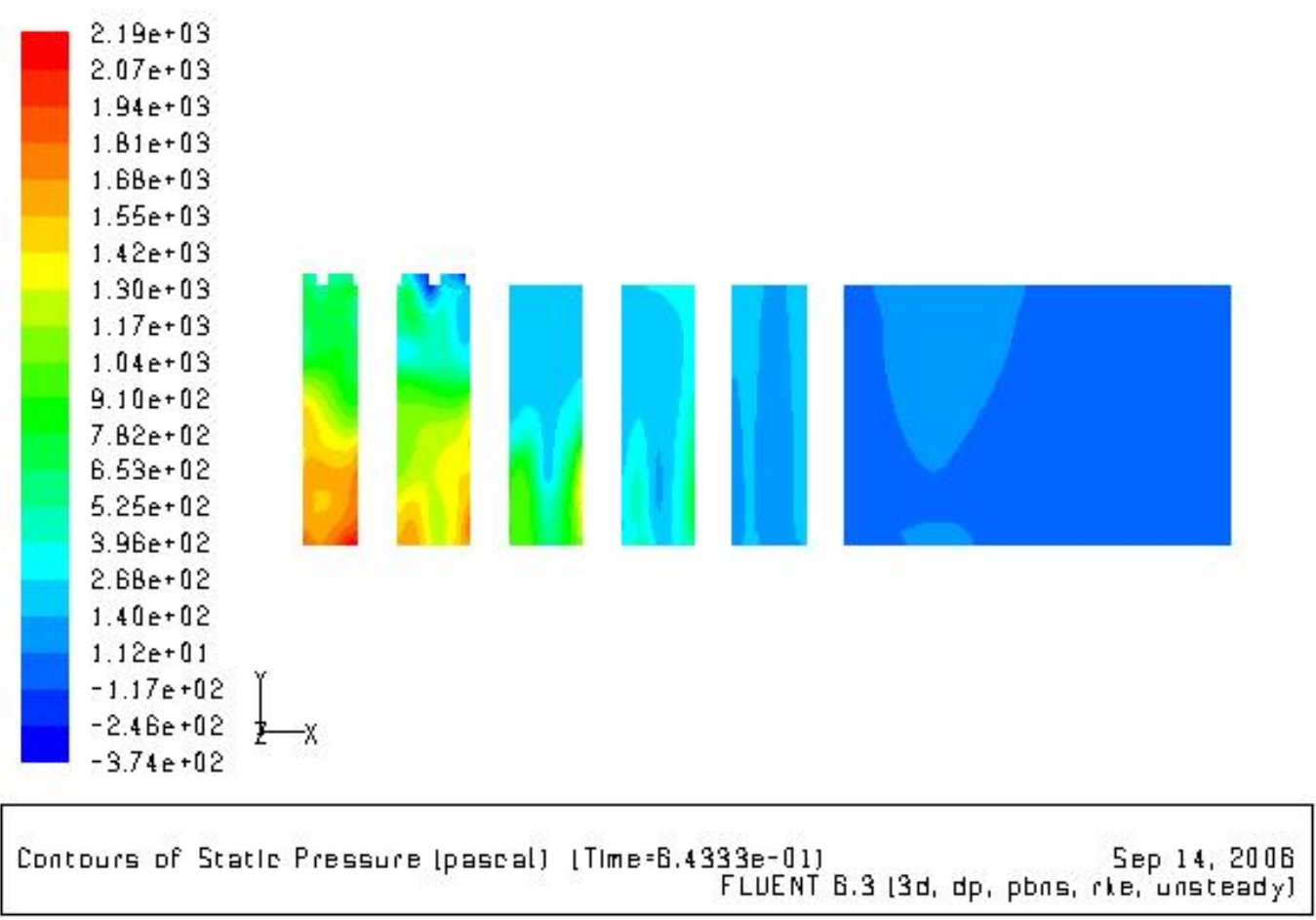

Figure 33. Computed contours of static pressure along the $\mathrm{x}-\mathrm{y}$ plane on the centerline $(\mathrm{z}=0)$.

TKE is defined by the relation:

$$
\text { tke }=0.5 *\left(\overline{u^{\prime} u^{\prime}}+\overline{v^{\prime} v^{\prime}}+\overline{w^{\prime} w^{\prime}}\right)
$$

where u', v', and w' are the three directional standard deviations in flow velocity. As shown in Figure 34, TKE is highest near the top of the model just beneath jet \#3. Upstream of post \#2, TKE is highest in the upper portion of the model. Between posts \#2 and \#4, TKE is highest in the lower portion of the model. TKE diminishes beyond post \#4. As a check of solution quality, TKE is zero at the walls.

Figure 35 shows turbulent intensity (TI) is the highest beneath the inlet jets. Beyond post \#2, TI is higher in the lower portion of the model than in the upper portion. In FLUENT ${ }^{\circledR}$, TI is computed as the RMS value of the local turbulent velocity fluctuations divided by the maximum velocity of $3.34 \mathrm{~m} / \mathrm{s}$.

The value of wall $y+$ should be reduced to be approximately equal to 1 at the first grid point away from the wall. 


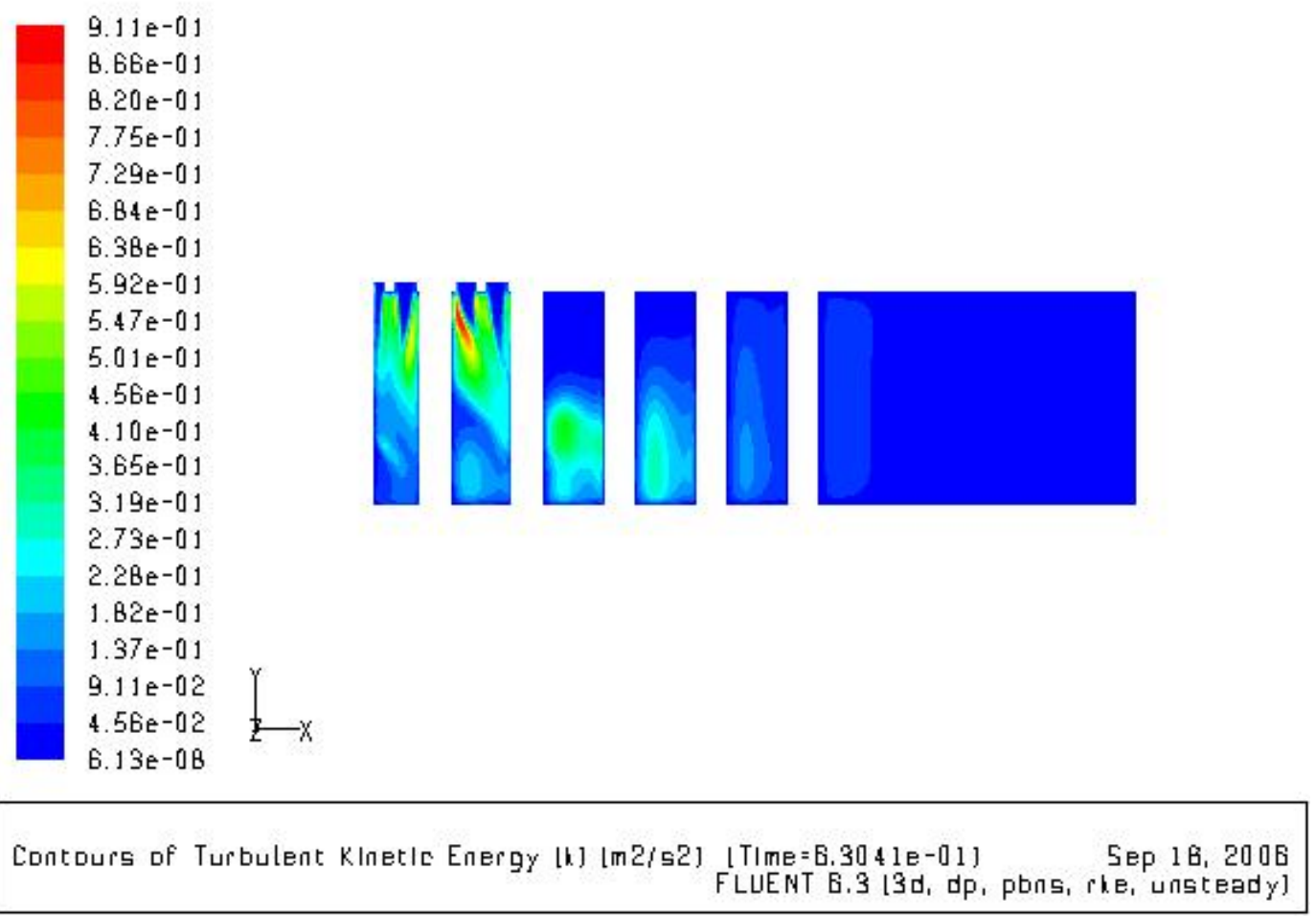

Figure 34. Computed contours of TKE on the $\mathrm{x}-\mathrm{y}$ plane along the centerline $(\mathrm{z}=0)$.

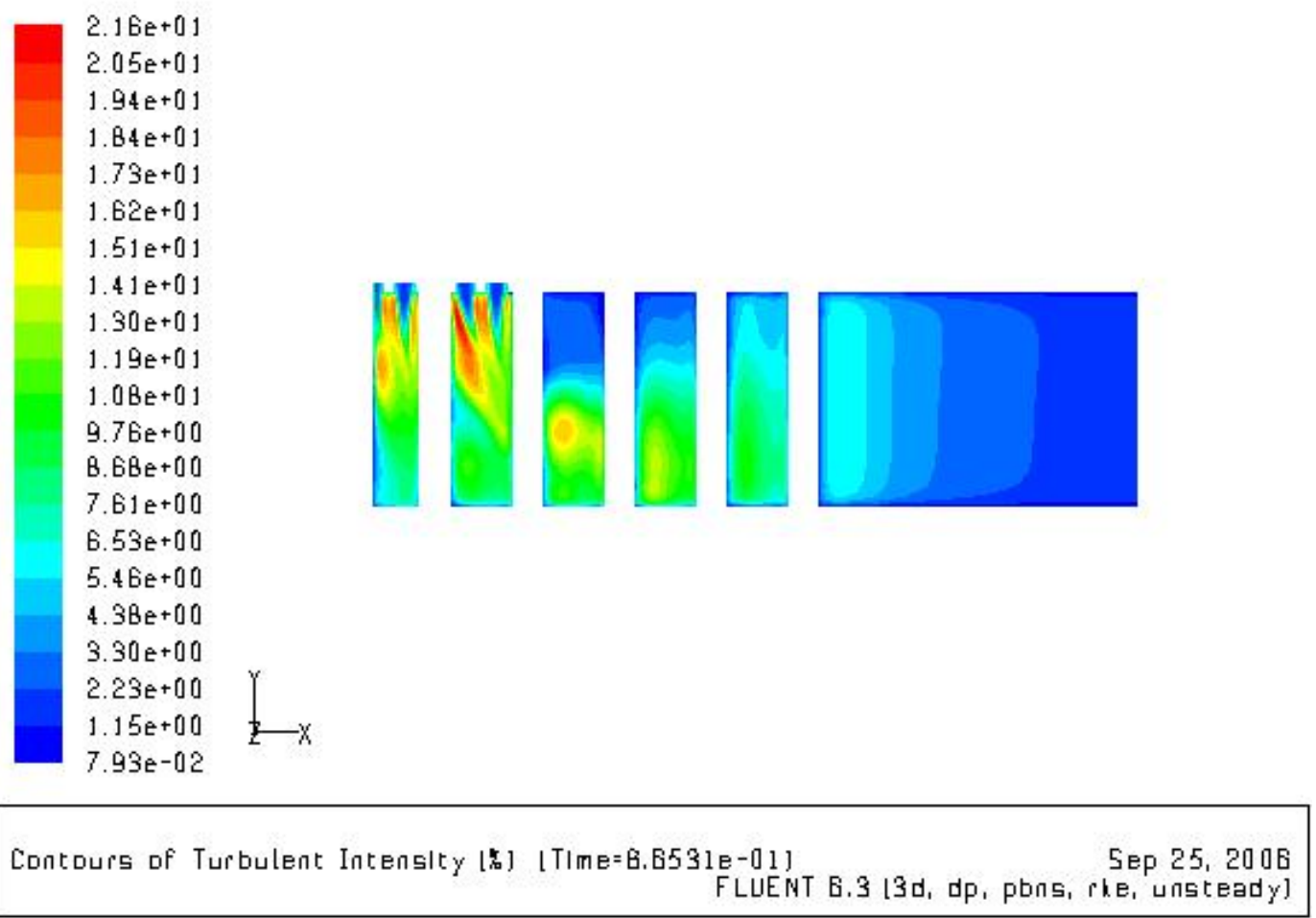

Figure 35. Computed contours of TI on the $\mathrm{x}-\mathrm{y}$ plane along the centerline $(\mathrm{z}=0)$. 
Figures 36 and 37 illustrate contours of $x$-velocity on $x-z$ planes located at $y=-0.07 \mathrm{~m}$ and $-0.15 \mathrm{~m}$, respectively. These $y$-locations are located at heights approximately $1 / 3$ and $2 / 3$ from the top of the plenum $(y=0)$. Note that the scales for the $x$-velocity ranges are different in these plots. Recirculation regions are indicated by negative $\mathrm{x}$-velocity. Regions of higher $\mathrm{x}$-velocity are present in the lower portion of the plenum. A wake forms on the downstream side of each cylindrical support post with various separation angles depending on flow speed and vertical location along the post. Slight asymmetries in the wake regions are observed.

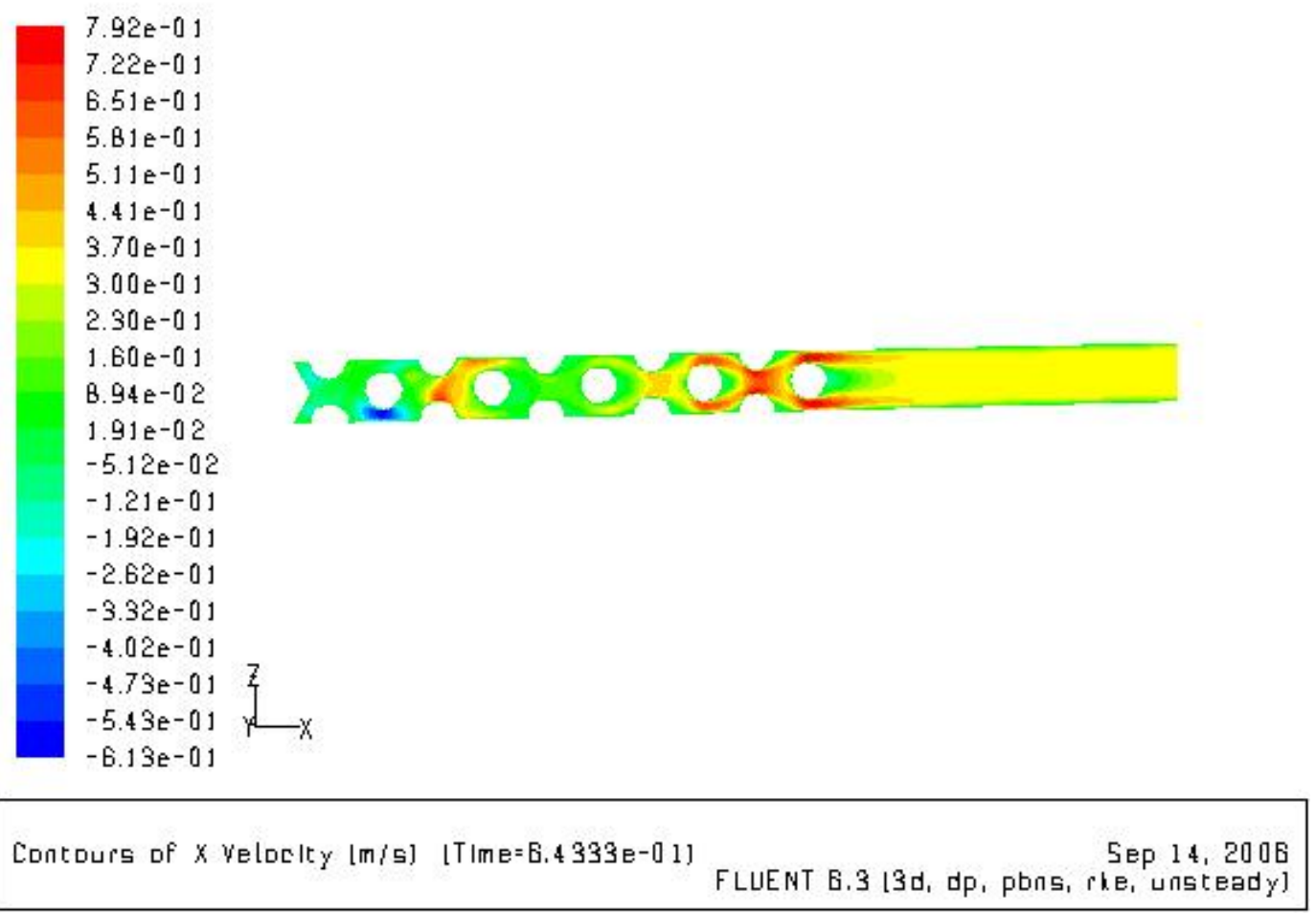

Figure 36. Computed contours of $x$-velocity along the $x-z$ plane at $y=-0.07 \mathrm{~m}$. 


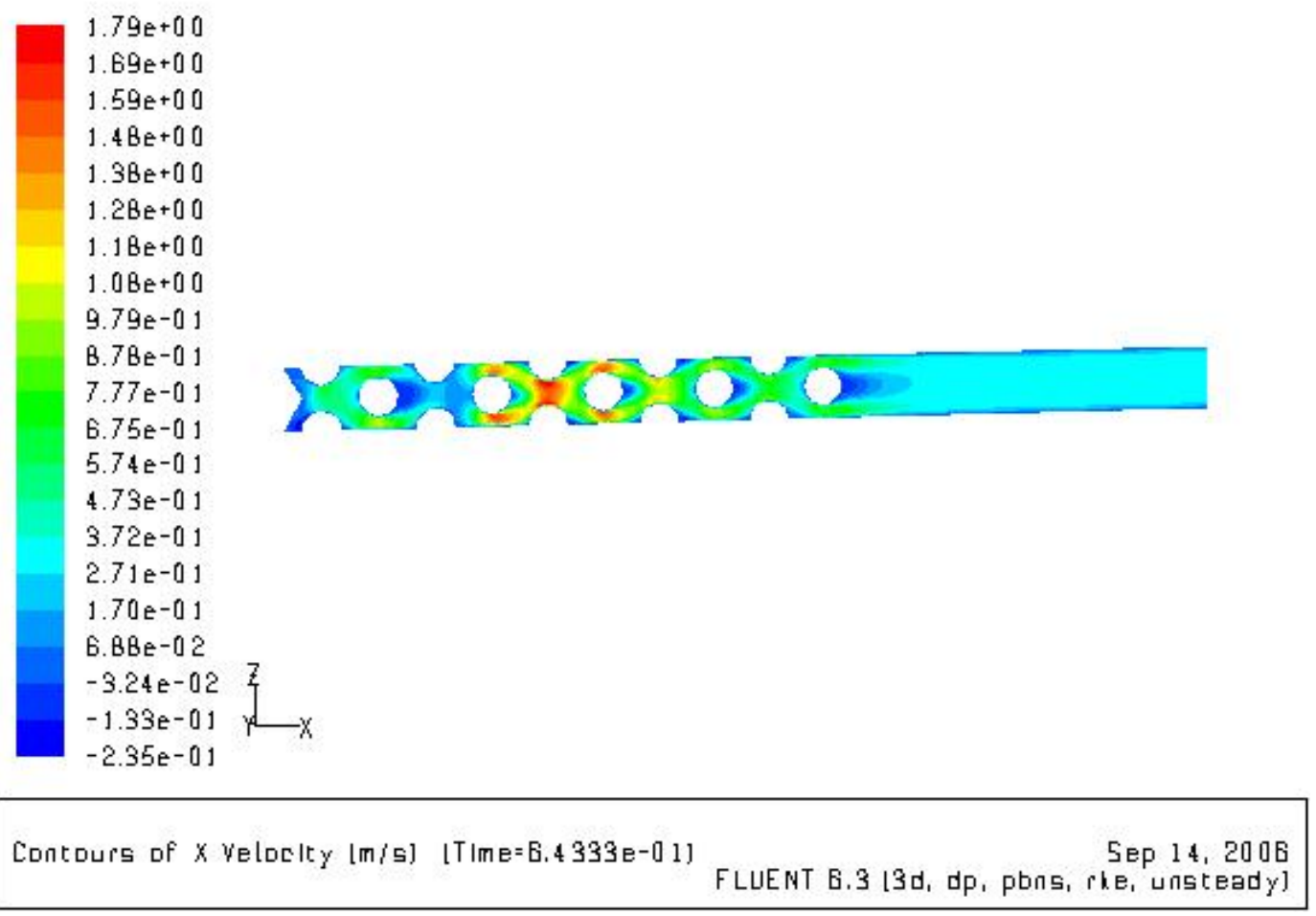

Figure 37. Computed contours of $x$-velocity along the $x-z$ plane at $y=-0.15 \mathrm{~m}$.

Figure 38 shows an expanded view of the x-velocity vectors in the vicinity of support post \#2 at a location $y=-0.15 \mathrm{~m}$ computed on the "super-fine" grid. The separation angle and recirculation length behind cylindrical support post $\# 2$ are approximately $140^{\circ}$ and $0.015 \mathrm{~m}$, respectively. The effect of the wake extends approximately half of a post diameter downstream. Small recirculation regions can be seen in the corners at the junction of the side walls and the half-cylinders. The flow can be seen to stagnate at the upstream face of the cylinders. 


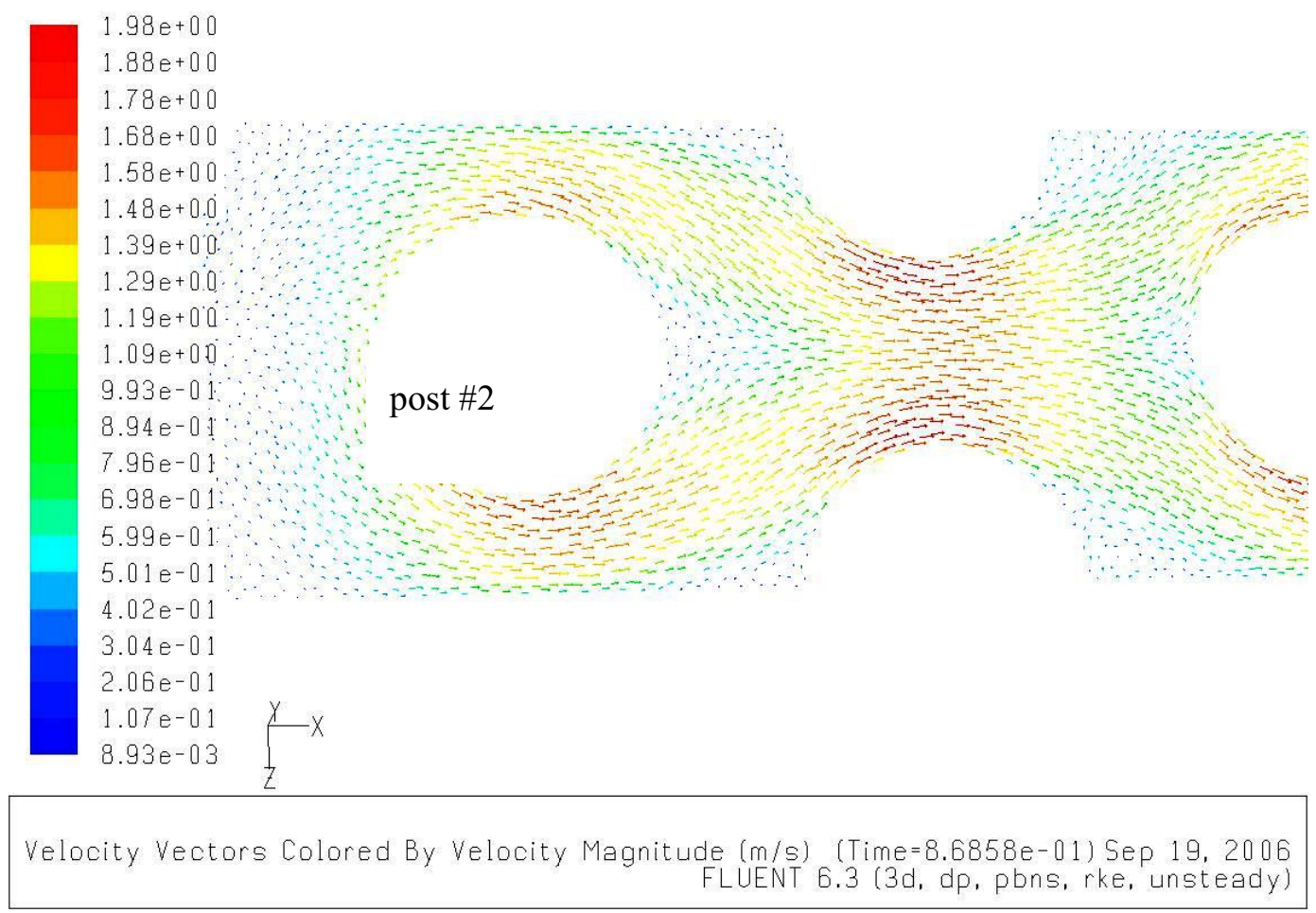

Figure 38. Computed $\mathrm{x}$-velocity vectors showing detail of wake region behind post $\# 2$.

Computationally it is undesirable to have backflow present at a boundary specified as a constant pressure outlet. The computational grid extended the outlet region by approximately $300 \mathrm{~mm}$ to eliminate backflow into the computational domain. Table 3 lists the minimum and maximum x-velocity present at the test section outlet and at the outlet of the extension. Figure 39 depicts computed contours of $x-$ velocity at the outlet plane of the test model. As seen, there is backflow into the plenum indicated by negative $x$-velocity, even with 4 jets operating. PIV data are not available at this location due to an obscuration in the test section. Figure 40 depicts computed contours of $\mathrm{x}$-velocity at the extended outlet. The $\mathrm{x}$-velocities at this location are all positive, indicating there is no backflow at the extended outlet.

Table 3. Computed maximum and minimum velocities at test section and computational outlets ("fine" unstructured grid).

\begin{tabular}{lcc}
\hline \multicolumn{1}{c}{$\mathbf{V}_{\mathbf{x}}(\mathbf{m} / \mathbf{s})$} & Minimum & Maximum \\
\hline Model outlet & -0.2283 & 0.6603 \\
Extended outlet & 0. & 0.3465 \\
\hline
\end{tabular}




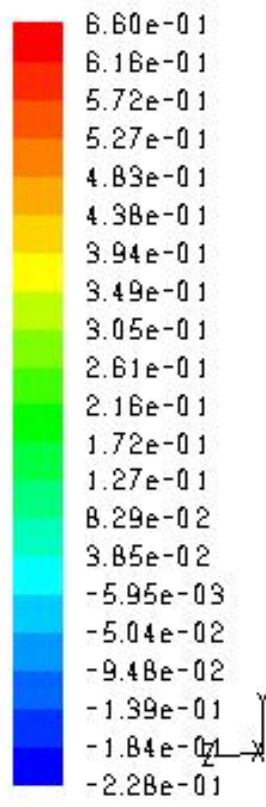

Contours of $X$ Velocity $[m / s]$ [TIme=6.1746e-[1]

Sep 14,2006

FLUENT E.3 [3d, dp, pbns, rhe, unsteady]

Figure 39. Computed contours of x-velocity at outlet of test model.
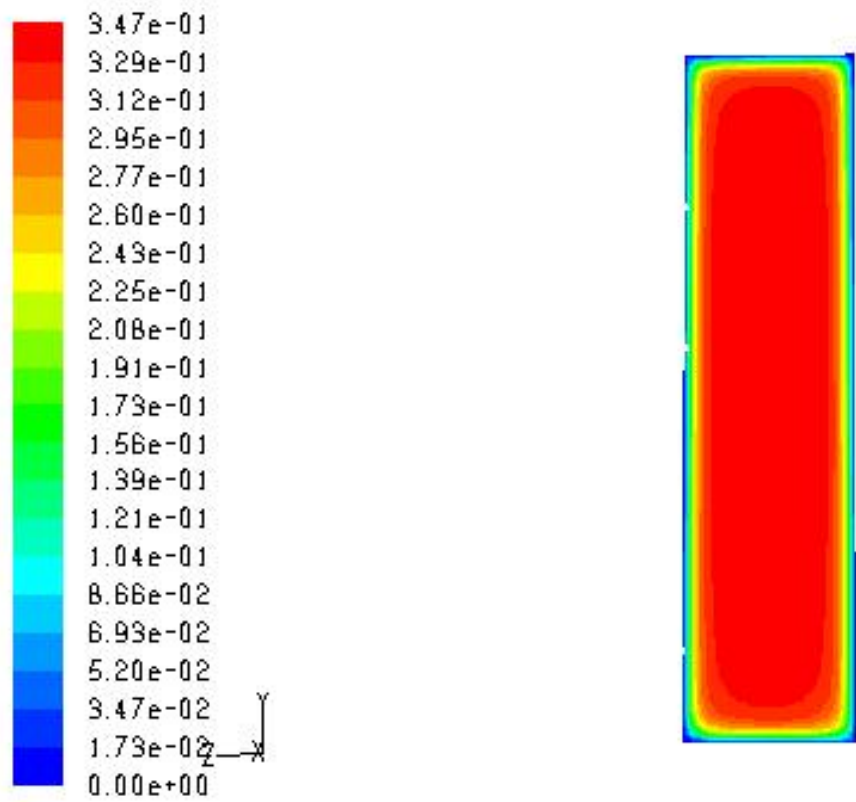

Contours of $x$ Velocity Im/s] ITIme=6.1746e-01]

Sep 14,2006

FLUENT E.3 [3d, dp, pbns, rhe, unsteady]

Figure 40. Computed contours of x-velocity at extended outlet. 
3.4.2.2 Line Plots of FLUENT ${ }^{\circledR}$ Results. Figure 41 illustrates the data planes for the plots shown in Figures 42-46. These figures illustrate the velocity magnitude at an instant of time. These solutions were performed on the "fine" unstructured grids for the following x-locations: $0.12022 \mathrm{~m}, 0.16850 \mathrm{~m}$, $0.19807 \mathrm{~m}$, and $0.26729 \mathrm{~m}$, and $\mathrm{y}$-locations: $-0.07 \mathrm{~m}$ and $-0.15 \mathrm{~m}$.

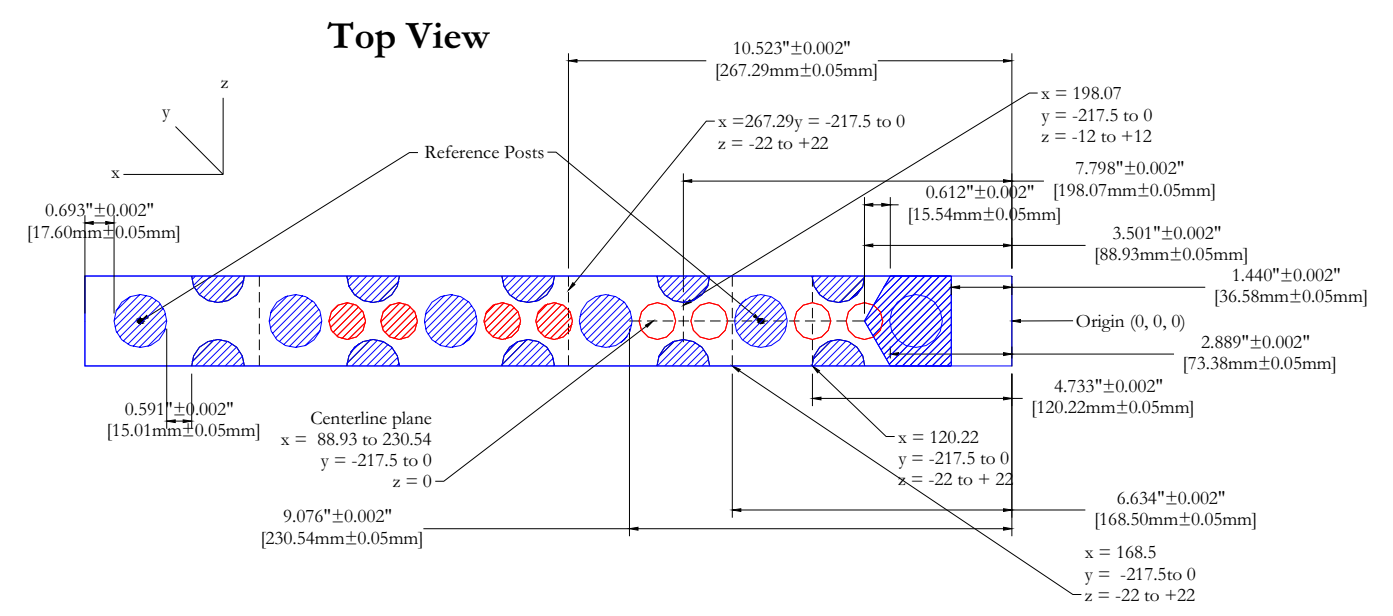

Figure 41. Data planes relative to model axis origin for data analysis.

Figure 42 shows the variation in velocity magnitude with z-location at two y-locations along the centerline of jet $\# 2$. The velocity is higher in the upper portion of the plenum. Figure 43 shows the variation of velocity magnitude with z-location on the aft side of post \#1. The dip in velocity near $\mathrm{z}=0$ is a consequence of the flow separation in the wake. Figure 44 shows the variation in velocity magnitude with z-location at the centerline of the second set of half-posts between posts \#1 and \#2 and jets \#3 and \#4. The velocity magnitude is higher in the upper portion of the plenum. The profile is more symmetric for $y=-0.15 \mathrm{~m}$. Figure 45 shows the variation in velocity magnitude with z-location slightly downstream of post \#2. The velocity magnitude is higher in the lower portion of the plenum. The effect of the wake can be seen as a velocity decrease around $\mathrm{z}=0$.

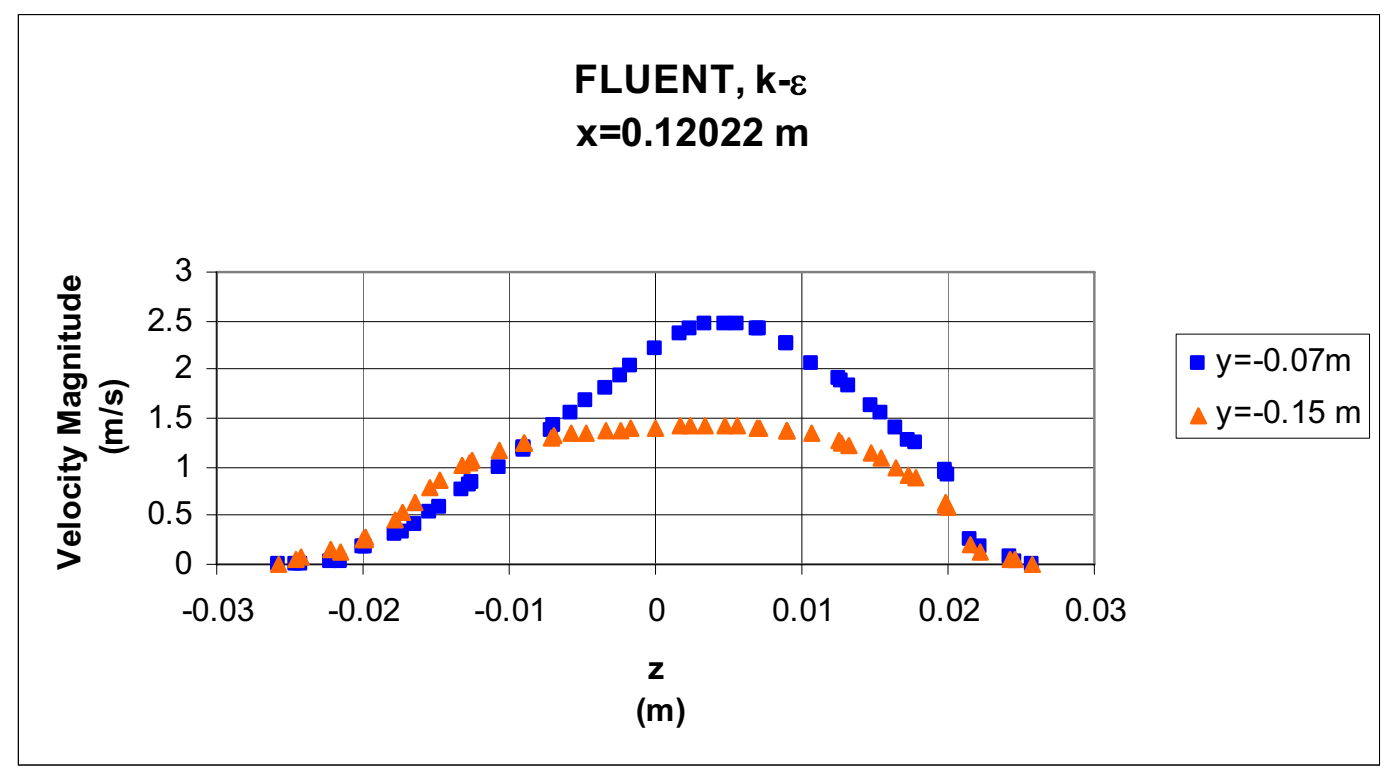

Figure 42. Variation of velocity magnitude along the plane $x=0.12022 \mathrm{~m}$ 


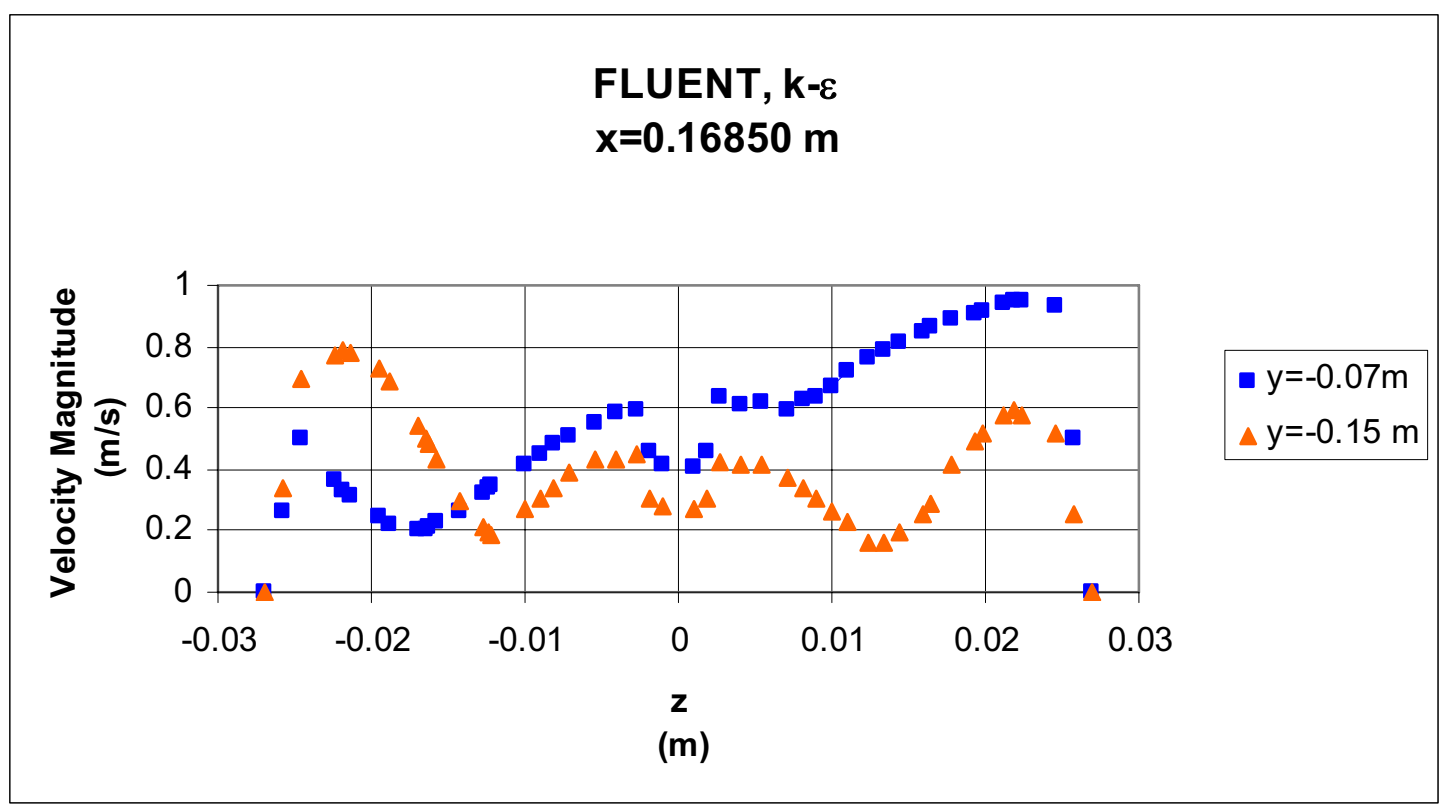

Figure 43. Variation of velocity magnitude along the plane $x=0.16850 \mathrm{~m}$

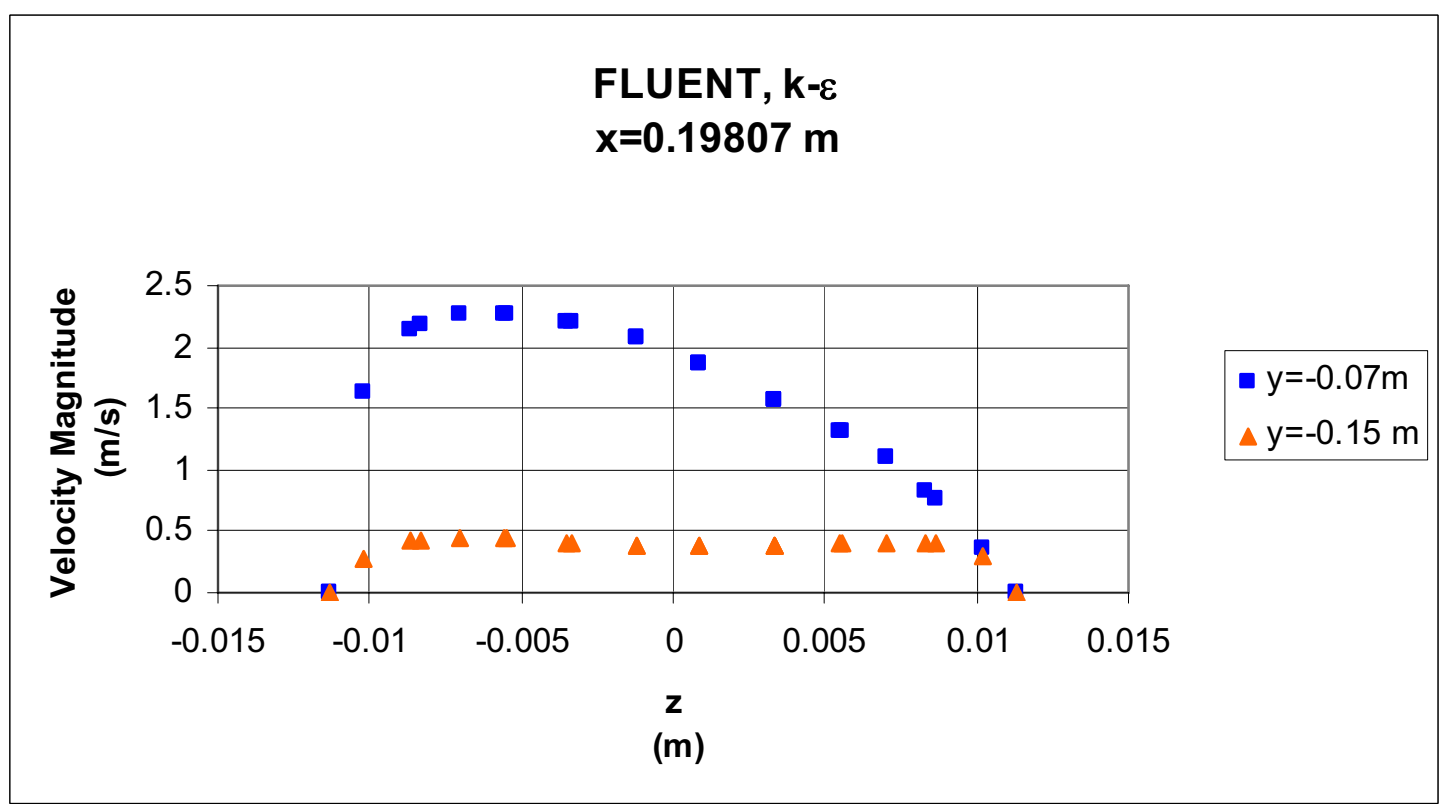

Figure 44. Variation of velocity magnitude along the plane $x=0.19807 \mathrm{~m}$ 


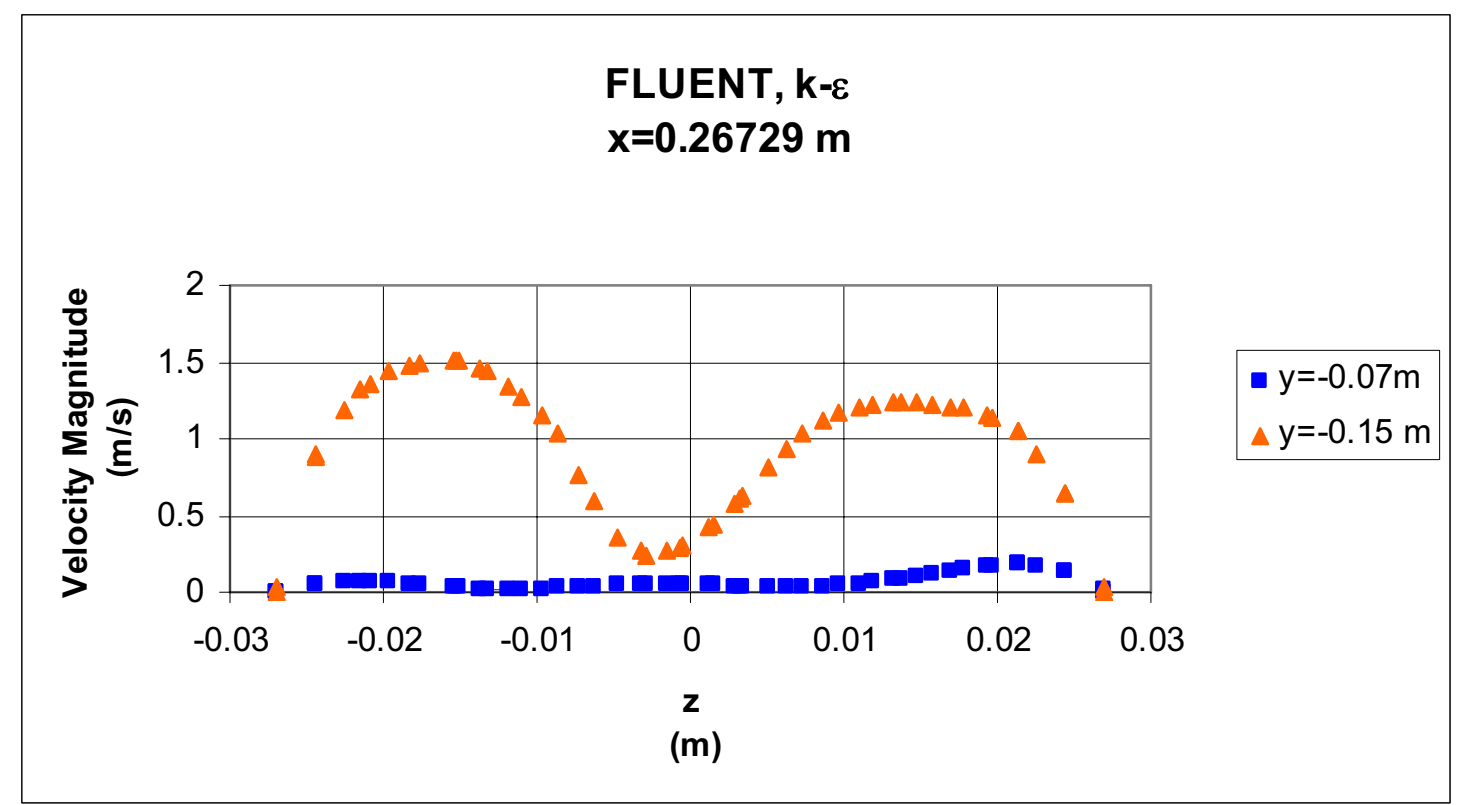

Figure 45. Variation of velocity magnitude along the plane $\mathrm{x}=0.26729 \mathrm{~m}$.

Figure 46 shows the variation in velocity magnitude along the model centerline $(\mathrm{z}=0)$ for two $\mathrm{y}$ locations. The high velocity at $y=-0.07 \mathrm{~m}$ in the upstream portion of the plenum is due to the inlet jet flow. The curve for $y=-0.15 \mathrm{~m}$ displays dips in velocity due to the wakes on the downstream side of the cylinders and the acceleration in velocity as the flow travels around the support posts and between the half-posts. The acceleration of the flow is due to the area reduction at these locations and can be explained by mass conservation [White, 2003]. Beyond the wake behind post \#5, the flow homogenizes and the velocity reaches a constant velocity of $\sim 0.35 \mathrm{~m} / \mathrm{s}$ from an $\mathrm{x}$-location approximately $0.76 \mathrm{~m}$ from the model origin to the end of the extended outlet.

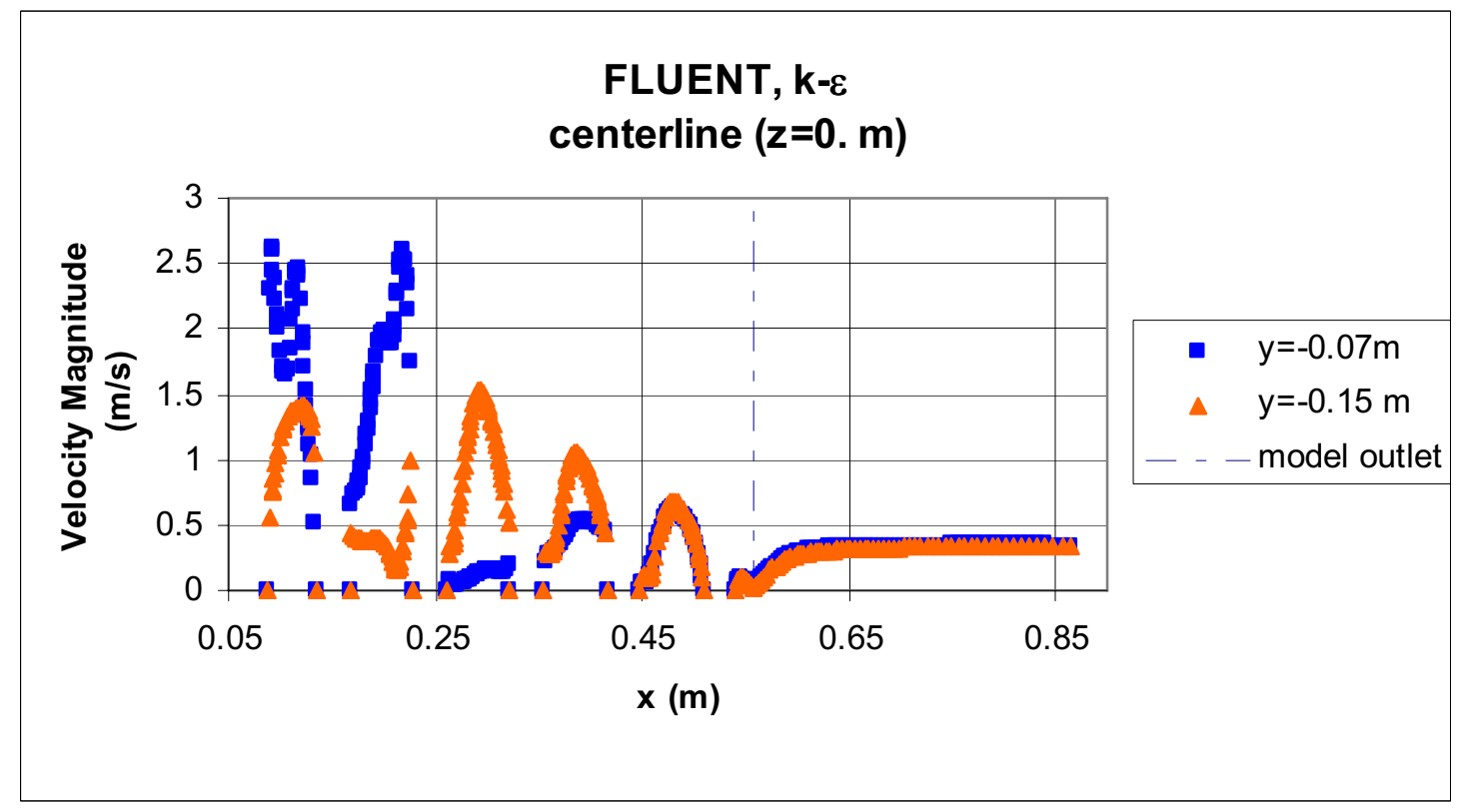

Figure 46. Variation of velocity magnitude along the flow centerline $(\mathrm{z}=0)$. 
The velocity profiles vary slightly with time. This is a result of the unsteadiness of the flow. Figure 47 shows the velocity along the data plane located at $x=0.26729 \mathrm{~m}$ and $\mathrm{y}=-0.15 \mathrm{~m}$ at two different snapshots in time. These results were produced on the "super-fine" grid and are more symmetric than the curves produced with results obtained using the "fine" grid (see Figure 45). The fact that the results are slightly different between the "fine" and "super-fine" grids indicates that the "fine" grid is not fine enough. The "super-fine" grid should be adapted based on flow gradients and the solution re-rerun. This process should be repeated until there are no further changes in the solution due to grid refinement.

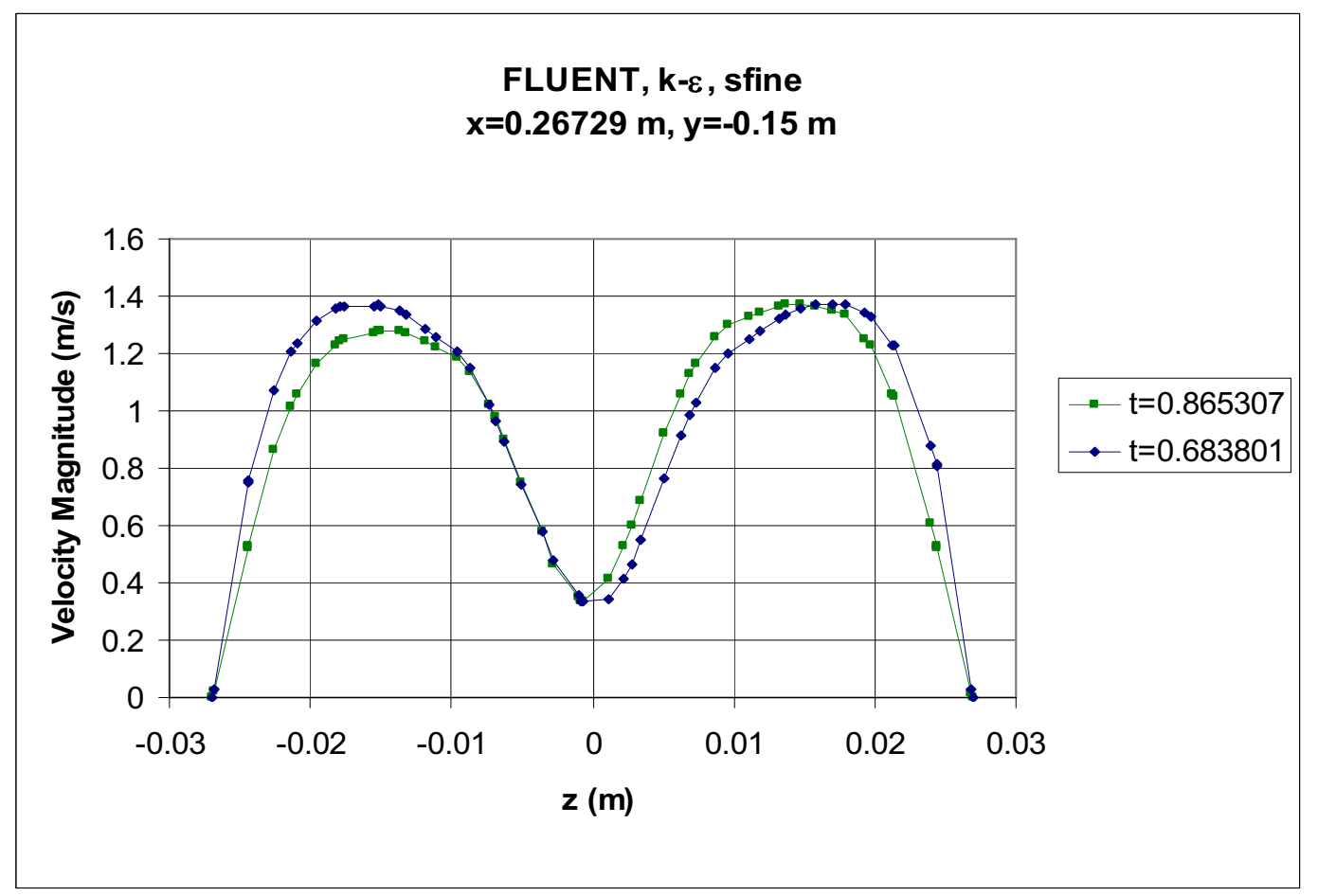

Figure 47. Variation of velocity at two different times.

\subsubsection{Flow Unsteadiness}

The flow is expected to exhibit unsteadiness due to the vortex shedding from the cylindrical support posts and also due to the turbulent fluctuations [Johnson, 2006]. Turbulent flow is characterized by fluctuations over a large range of scales. Fluctuations at different scales interact with each other and exchange momentum and energy. Turbulent transport therefore can be seen as "diffusive" in nature. As a result of mixing between turbulent eddies of all sizes, turbulent transport is a much more effective process than molecular transport, which is a very slow process on a macroscopic scale.

Non-turbulent fluctuations can be characterized by the non-dimensional frequency of fluctuation (i.e., the Strouhal number). From dimensional analysis for an oscillating flow, it can be shown that:

$$
\frac{\mathrm{w}_{\mathrm{v}} \mathrm{d}}{\mathrm{V}}=\mathrm{fn}\left(\frac{\mathrm{Vd}}{\mathrm{v}}\right)
$$

or 


$$
\mathrm{St}=\mathrm{fn}\left(\mathrm{Re}_{\mathrm{d}}\right)
$$

where the post Reynolds number, $\mathrm{Re}_{\mathrm{d}}$, of the flow approaching the cylinder is given by the ratio of inertia forces to viscosity forces

$$
\operatorname{Re}_{\mathrm{d}}=\frac{\mathrm{V}_{\mathrm{x}} \mathrm{d}}{\mathrm{v}}
$$

and the Strouhal number, St, is given by the ratio of the oscillation to the mean speed [White, 2003]

$$
\mathrm{St}=\frac{\mathrm{w}_{\mathrm{v}} \mathrm{d}}{\mathrm{V}_{\mathrm{x}}}
$$

In these equations

$\mathrm{V}_{\mathrm{x}}=$ flow velocity in the $\mathrm{x}$-direction, $\mathrm{m} / \mathrm{s}$

$\mathrm{w}_{\mathrm{v}}=$ vortex shedding frequency, $\mathrm{Hz}$

$\mathrm{d}=$ diameter of support post, $0.03175 \mathrm{~m}$

$v=$ kinematic viscosity of the fluid, $1.41 \times(10)^{-5} \mathrm{~m}^{2} / \mathrm{s}$.

Vortex shedding occurs for $10^{2}<\operatorname{Re}_{\mathrm{d}}<10^{7}$, and the Strouhal number remains approximately constant $(\approx 0.2)$ over this range of Reynolds numbers [White, 2003]. The frequency of the vortices shed from the cylindrical support posts is approximated by the following analysis:

$$
w_{v}=\frac{V_{x} S t}{d}
$$

Using the maximum flow velocity in the $\mathrm{x}$-direction, $\mathrm{V}_{\mathrm{x}}$, a maximum vortex shedding frequency of $11.3 \mathrm{~Hz}$ and a post Reynolds number, $\mathrm{Re}_{\mathrm{d}}$, of 4037 is obtained. Based upon the work by Smith et. al (2006), the flow in this regime is expected to be turbulent outside and laminar inside the boundary layer on the posts. To capture the time progression of vortices shed from the cylinder, the system response of the data acquisition system should be at least $1 \mathrm{kHz}$. At this Reynolds number the flow is turbulent and will exhibit much higher frequency turbulent fluctuations superimposed on the flow [Johnson, 2006].

The results do not exhibit the formation of a vortex street. Rather, standing wakes with timevarying oscillations on the downstream sides of the cylindrical support posts are witnessed. This may be due to numerical effects, such as insufficient grid resolution or excessive numerical damping, or to physical effects caused by placing the cylindrical posts in a confined channel rather than in a free-stream flow [Khan, et. al, 2004]. This result requires further investigation. An asymmetric perturbation may have to be induced in the flow to initiate vortex shedding. However, vortex shedding downstream of the cylindrical support posts is also not seen in the preliminary experimental data. The amount of seeding used for the PIV measurements will be increased to improve the data quality, which may help us see vortices if they are indeed present in the flow. Ensemble averaging the experimental data may average out any unsteadiness in the flow. The time interval at which the instantaneous data is currently acquired by the PIV instrumentation is too large to resolve fluctuations in the flow due to non-stationary and turbulence effects. 


\subsection{Future Work}

Areas for further study include:

- Comparisons between results to guide further experiments and refinements in the final CFD simulations. This work will also be used to guide the production CFD simulations of the entire lower plenum. Quantitative comparison between the CFD solutions and the experimental data are necessary. At the date this report was written, the final data are not available.

- Application of other turbulence closure models with the FLUENT ${ }^{\circledR}$ URANS approach, including the $\mathrm{k} \sim \omega$ or RSM models. The use of LES may be necessary to resolve detailed transient flow behavior.

- Determination of the most accurate and efficient numerical schemes (e.g., SIMPLEC vs. PISO, QUICK vs. MUSCL, etc.).

- Extension of the NPHASE work to include URANS solutions. The NPHASE solutions should be run with all four inlet jets operational, so that the results can be compared to the FLUENT $^{\circledR}$ results and the experimental data.

- Evaluation of the effect of different initial conditions when using the $\mathrm{k} \sim \varepsilon$ turbulence model. Also, adjustment of $\varepsilon$ to determine if there is an effect.

- Further investigation of vortex shedding. Asymmetric perturbation of the flow may be necessary to initiate the shedding.

- Completion of the grid independence study. Adaptation and/or manual adjustment/regeneration of the grids is required to achieve the desired $y+$ value. A solution performed with grids constructed of hexagonal or polyhedral cells should converge faster.

- Rigorous evaluation of the error in the numerical solution [Celik, 2006 ;Roache, 1986].

\subsection{Summary}

This report documents the progress of turbulent CFD predictions for a section of the VHTR lower plenum using the NPHASE and FLUENT ${ }^{\circledR}$ codes. To date, the NPHASE simulations have focused on RANS steady-state solutions and the standard $\mathrm{k} \sim \varepsilon$ turbulence model with 2 inlet jets operating. FLUENT $^{\circledR}$ simulations have been performed for unsteady flow using the realizable $\mathrm{k} \sim \varepsilon$ turbulence model with 4 inlet jets operating. These results should be treated as preliminary and used to guide the final CFD computations and experiments. Areas for further study have been outlined. Comparison with relevant experimental data is necessary to validate the CFD models.

Data obtained at one instant of time from the FLUENT $^{\circledR}$ solutions were given for $y$-locations approximately $1 / 3$ and $2 / 3$ from the top of the plenum and at four $x$-locations at the upstream end of the model. The largest gradients in velocity, pressure, and turbulent quantities occur in the region below the inlet jets. A Reynolds number calculation confirms that turbulent flow is present in the lower plenum model. Line plots performed for results at various time-steps indicate that the flow quantities fluctuate with time. Contours of $\mathrm{x}-, \mathrm{y}-, \mathrm{z}$-velocity; velocity magnitude; static pressure; TKE; and TI show that the flow is highly three-dimensional. Although the model is symmetrical, the flow exhibits asymmetry. An 
unsteady wake forms on the downstream side of each cylindrical support post with various separation angles depending on flow speed and vertical location along the post.

The experience gained from performing these CFD simulations on a sub-section of the VHTR lower plenum for a constant property fluid will provide the basis for modeling the entire lower plenum with turbulent mixing of hot gas jets. To be considered applicable for modeling more complicated flows, the computational models must first demonstrate the ability to predict a simplified case with reasonable accuracy. 


\section{References}

Antal, S. P., S. M. Ettorre, R. F. Kunz, and M. Z. Podowski, "Development of a Next Generation Computer Code for the Prediction of Multicomponent Multiphase Flows," International Meeting on Trends in Numerical and Physical Modeling for Industrial Multiphase Flow, Cargese, France, September 27-29, 2000.

ASME Journal of Fluids Engineering, 144, June 1996, p. 427.

Benhamadouche, S., and D. Laurence, "LES, coarse LES, and transient RANS comparisons on the flow across a tube bundle," International Journal of Heat and Fluid Flow, 24(4), 2003, p. 470-479.

Celik, I., "Numerical Uncertainty in Fluid Flow Calculations: Needs for Future Research," ASME Journal of Fluids Engineering, 115, 1993, p. 194-195.

Celik, I., Procedure for Estimation and Reporting of Discretization Error in CFD Applications, Mechanical and Aerospace Engineering Department, West Virginia University, 2006.

Condie, K.G., G. E. McCreery, H. M. McIlroy, and D. M. McEligot, Development of an Experiment for Measuring Flow Phenomena Occurring in a Lower Plenum for VHTR CFD Assessment, INL/EXT05-00603, Idaho National Laboratory, September 2005.

FLUENT, version 6.2.16, FLUENT Inc., 10 Cavendish Court, Centerra Resource Park, Lebanon, NH, 03766, 2005.

Gallaway, T. L., VHTR Lower Plenum Computational Fluid Dynamics Simulations using the NPHASE Code, INL/EXT-06-11780, Idaho National Laboratory, September 2006.

Gambit User Guide, version 2.2.30, FLUENT Inc., 10 Cavendish Court, Centerra Resource Park, Lebanon, NH, 03766, 2005.

General Atomics, Gas Turbine - Modular Helium Reactor (GT-MHR) Conceptual Design Description Report, Doc. 910720, Rev. 1, July, 1996.

Gridgen User Manual, Version 15.09, Pointwise, Inc., 213 South Jennings Avenue, Fort Worth, TX, 76104-1107, 2006.

Hanjalic, K., "Will RANS survive LES? A View of Perspectives," Journal of Fluids Engineering, 127, p. 831-839, 2005.

Hassan, Y. A., and H. R. Barsamian, "Tube bundle flows with the large Eddy simulation technique in curvilinear coordinates," International Journal of Heat and Mass Transfer, 47(14-16), 2004, 30573071 .

Johnson, R. W., R. J. and MacKinnon, "Equivalent versions of the QUICK scheme for finite-difference and finite-volume numerical methods," Communications in Applied Numerical Methods, 8(12), p. 841-848, 1992.

Laurence, D., personal communication, March 2005. 
Johnson, R. W., Validation Studies for Numerical Simulations of Flow Phenomena Expected in the Lower Plenum of a Prismatic VHTR Reference Design, INL/EXT-05-00787 Idaho National Laboratory, September, 2005.

Johnson, R. W., "Modeling Strategies for Unsteady Turbulent Flows in the Lower Plenum of the VHTR," CFD4NRS Workshop on Benchmarking of CFD Codes for Application to Nuclear Reactor Safety, Garching, Munich, Germany, 5-7 September 2006.

Khan, W. A., J. R. Culham, and M. M. Yovanovich, "Fluid Flow and Heat Transfer from a Cylinder between Parallel Planes," Journal of Thermophysics and Heat Transfer, 18(3), July-September 2004.

MacDonald, P. E., et al., NGNP Preliminary Point Design, Results of the Initial Neutronics and Thermalhydraulic Assessments, Rev. 1, INEEL/EXT-03-00870, Idaho National Engineering and Environmental Laboratory.

Moulinec, C., J. C. R. Hunt, and F. T. M. Nieuwstadt, "Disappearing Wakes and Dispersion in Numerically Simulated Flows Through Tube Bundles," Flow, Turbulence and Combustion, 73(2), 2004, 95-116.

Roache, P. J., K. N. Ghia, and F. M. White, "Editorial Policy Statement of the Control of Numerical Accuracy," ASME Journal of Fluids Engineering, 108, 1986, p. 2.

Roache, P. J., Verification and Validation in Computational Science and Engineering, Hermosa Publishers, 1998.

Rollet-Miet, P., D. Laurence, and J. Ferziger, "LES and RANS of Turbulent Flow in Tube Bundles," International Journal of Heat and Fluid Flow, 20(3), 1999, 241-254.

Rumsey, C. L., B. A. Pettersson Rief, and T. B. Gatski, "Arbitrary Steady-State Solutions with the K $\sim \varepsilon$ Model," AIAA Journal, 44(7), July 2006.

Simonin, O., and M. Barcouda, "Measurements and Prediction of Turbulent Flow Entering a Staggered Tube Bundle," in: 4th International Symposium on Applications of Laser Anemometry to Fluid Mechanics, Lisbon, Portugal, Paper 5.23, 1988

Simonin, O., and M. Barcouda, "Measurements of Fully Developed Turbulent Flow across Tube Bundle," Proceedings of the Third International Symposium on Applications of Laser Anemometry to Fluid Mechanics, Lisbon, Portugal, 1986, p. 21.5.1-21.5.5.

Smith, B. L., J. J. Stepan, and D. M. McEligot, "Velocity and Pressure Measurements Along a Row of Confined Cylinders," FEDSM2006-98186, Proceedings of ASME-FED 2006, 2006 ASME Fluids Engineering Summer Conference, Miami, FL, July 17-20, 2006.

White, F. M., Fluid Mechanics, $5^{\text {th }}$ Edition, McGraw Hill, 2003, p. 312-313. 
This page intentionally left blank. 


\section{Appendix}

\section{User-Defined Function (UDF) to Compute Time-Averaged Correlations}

\#include "udf.h"

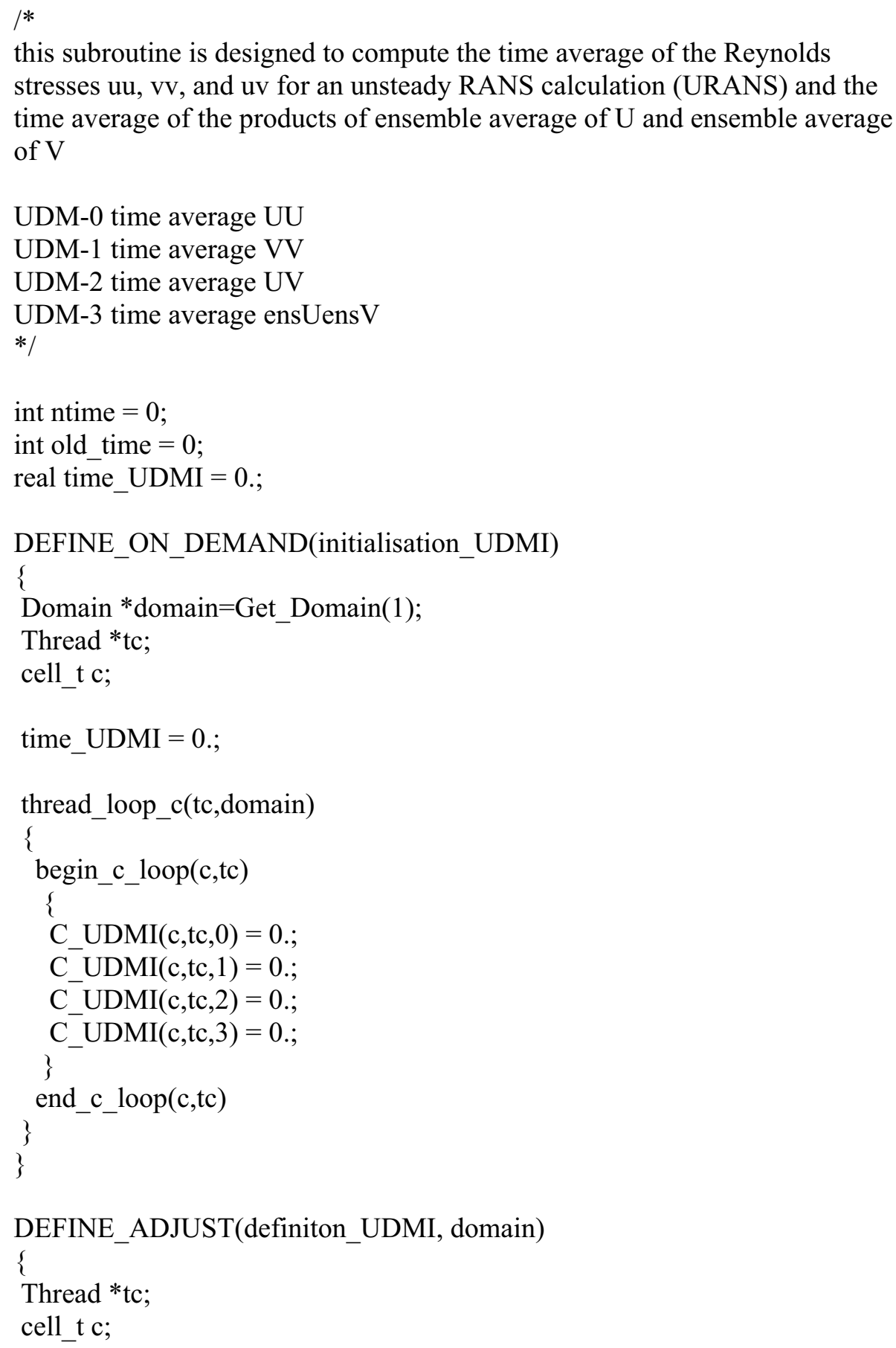




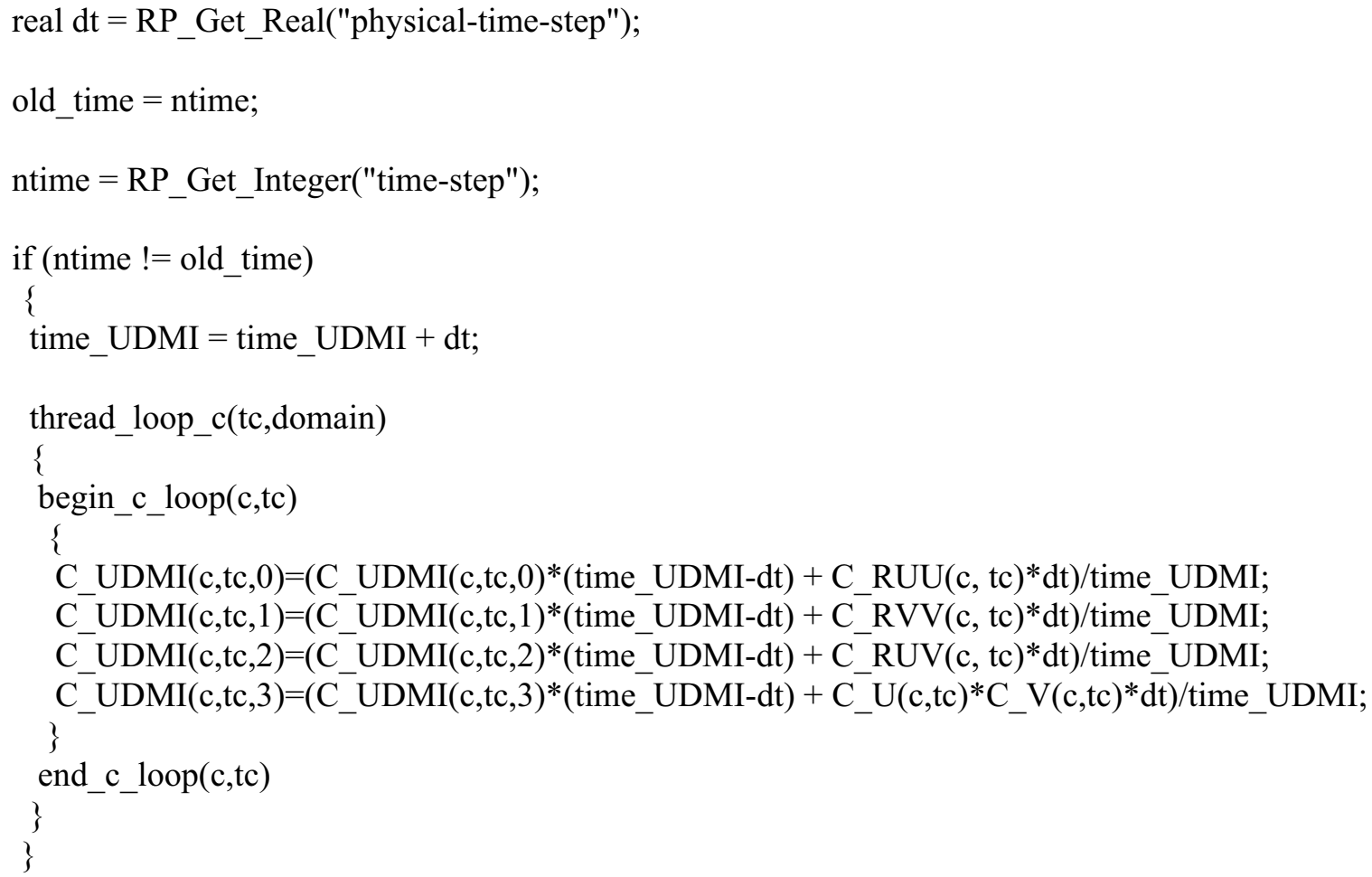

